\title{
INL Waste Management Program Assessment Report
}

\author{
Delbert Randall Allen
}

December 2020

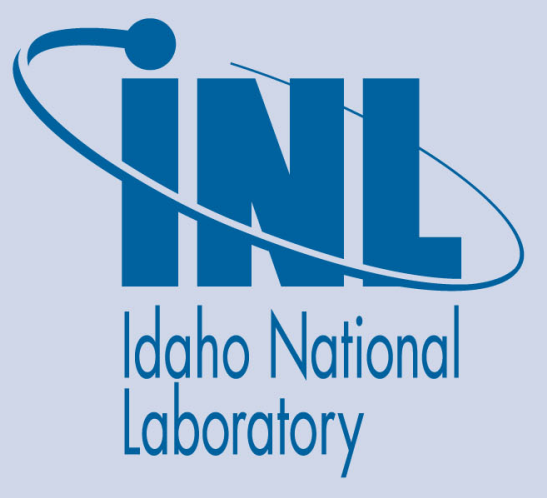

The INL is a U.S. Department of Energy National Laboratory operated by Battelle Energy Alliance 
INL/MIS-20-60702-Revision-0

\title{
INL Waste Management Program Assessment Report
}

\author{
Delbert Randall Allen
}

December 2020

Idaho National Laboratory Idaho Falls, Idaho 83415

http://www.inl.gov

Prepared for the U.S. Department of Energy Office of Environmental Management Under DOE Idaho Operations Office Contract DE-AC07-05ID14517 


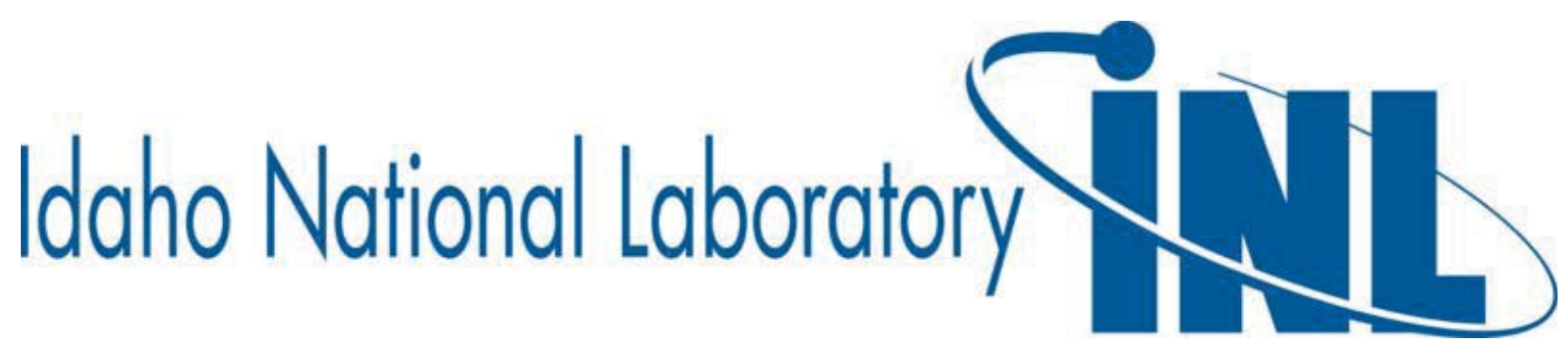

Independent Assessment of INL

Compliance with Nevada National Security Site Waste Acceptance Criteria NNSSWAC Requirements

Independent Assessment

\author{
Performed by \\ INL Quality \\ Battelle Energy Alliance, LLC \\ Assessment Number: ASMT 2020-0616
}

November 19, 2020 


\section{Independent Assessment of Compliance with Nevada National Security Site Waste Acceptance Criteria NNSSWAC November 2016 Requirements}

Lead Assessor:

JEFFERY FLUCKIGER (Affiliate) $\begin{aligned} & \text { Digitally signed by JEFFERY FLUCKIGER (Affiliate) } \\ & \text { Date: } 2020.11 .30 \text { 15:43:44-07'00' }\end{aligned}$

J. J. Fluckiger, Lead Auditor

Quality Training and Support Manager

Battelle Energy Alliance, LLC

Assessment Approval:

McClung, Bradley D

(MCCLBD)

Digitally signed by McClung, Bradley D

(MCCLBD)

Date: 2020.12 .01 09:09:08 -05'00'

B. D. McClung

Quality Research and Special Projects Manager

Battelle Energy Alliance, LLC

$\begin{array}{ll}\begin{array}{l}\text { KENT MILLER } \\ \text { (Affiliate) }\end{array} & \begin{array}{l}\text { Digitally signed by KENT MILLER } \\ \text { (Affiliate) }\end{array} \\ \text { Date: 2020.12.02 05:20:41 -07'00' }\end{array}$

K. L. Miller

Date: 2020.12.02 05:20:41 -07'00'

Director, Environmental Support Services

Battelle Energy Alliance, LLC
Date

Date

Date 


\section{EXECUTIVE SUMMARY}

In accordance with section 5.10 of DOE/NV--325-16-00, the Nevada National Security Site (NNSS) Waste Acceptance Criteria (WAC), this independent assessment has been performed to verify INL Waste Management Program compliance with NNSS WAC requirements and to promote process improvement.

\section{ASSSESSMENT ACTIVITIES}

The NNSS WAC program audit checklists obtained from NNSS were used to ensure all aspects of Waste Certification Program activities were included and evaluated. The checklists cover the topics of Quality Assurance, Radiological Waste and Chemical Characterization, Traceability, and Transportation.

This assessment was conducted during a period of controlled work conditions due to the Covid19 pandemic. Therefore, this assessment was performed primarily as a records/documentation review using completed FY 2020 shipment files. Approximately 25 shipments, with documentation available for review, had been completed at the time the assessment was initiated. A sampling of approximately 10\%, (3 completed shipment files) was selected for review. The sampling represented two different waste streams and included both boxes and drums as waste containers. One live shipment of a cask was also observed.

Assessment activities focused on evaluation of program control documents and observation of completed shipment records that demonstrate adherence to the program. Interviews and conversations with waste management personnel were also included in the assessment. Documents reviewed and activities observed are recorded in the attached checklists. Implementation of the requirements was found to be compliant.

The Assessment Plan is included as Appendix A to this Report. Documentation of personnel observed during work performance is included as Appendix B. A listing of Waste Management Program procedures and forms is included in Appendix C. Verification of program or procedure adequacy together with observed objective evidence demonstrating process implementation are included in the response sections of the checklists which are Appendices D through $\mathrm{H}$.

\section{CONCLUSION}

Overall, based on the objective evidence and observations of work performed, the assessors determined that BEA, in conjunction with WGS, has an effective and compliant Waste Management program, consisting of procedures, specialized forms, checklists, and qualified personnel, to ensure compliance with regulatory and contractual waste management requirements. The BEA Waste Management Program is compliant with the NNSS WAC.

One identified Condition was presented to management. The Condition was minor and was corrected during the assessment. Details are included in the body of this report. Several observations or recommendations for improvement have also been provided (see below).

Overall Performance Rating: Effective

This rating is based on objective evidence of program adequacy and documentation of program implementation in accordance with the criteria provided within DOE/NV--325-16-00, the NNSS WAC, issued November 2016. 


\section{ASSESSMENT PURPOSE AND SCOPE}

The purpose of this independent assessment was to evaluate the INL Battelle Energy Alliance, LLC (BEA) Waste Management Program and implementation against the requirements specified in DOE/NV--325-16-00, the NNSS WAC, issued November 2016.

The primary focus of this assessment was on requirements from the NNSS WAC. Per INL PLN17522 "Quality Assurance Program Plan for Low-Level, Mixed Low-Level and Hazardous Waste Management/Waste Certification”, the NNSSWAC program audit checklists obtained from NNSS were used. Specifically:

1) NNSSWAC DOE/NV-325-16-00 Quality Assurance Checklist

2) NNSSWAC 325-16-00 Radiological Waste Characterization Checklist

3) NNSSWAC DOE/NV--325-16-00 Chemical Characterization Checklist

4) NNSSWAC 325-16-00 Waste Traceability Checklist

5) NNSSWAC 325-16-00 Waste Transportation Assessment Checklist

To accomplish this assessment, a review of the completed checklists from the assessments performed over the last two years was conducted. During this initial review, all programmatic responses to checklist questions were verified as accurate or were updated when it was discovered that procedures or processes had been revised. Implementation of program controls was verified through review of completed shipment files for waste that had been shipped to NNSS during FY 2020, and through on-site observation of a shipment from the Advanced Test Reactor Complex to NNSS.

Additionally, it was observed that in the completed assessment report from 2018, a finding had been noted. The finding was stated as follows:

PDD-13000 Section 6.17 requires "Quality assurance records shall furnish documentary evidence that items or activities meet specified quality requirements". Contrary to that requirement, forms were identified that had sections not completed. Examples include:

a. Form 435.B04, NNSS Driver Briefing for Shipment NEL18014 was incomplete in the "Tritium" portion of the form. This condition was self-identified by the WCO during the course of this assessment.

b. Form 435.79, Container Information and Closure Checklist for CO225 Container MFC180150, Section V-Isotopic Information, was blank for the outer container.

As follow up on the effectiveness of corrective actions for the finding listed above, completed documentation for the shipments selected was reviewed. Reviews revealed that most forms had been completed properly. In one instance a duplicate of a form was used to capture two separate signatures. In this case both forms were included in the shipping file which gave the appearance that neither form was properly completed. Additionally, two 435.88 forms, that were presented to the assessment team for review, did not have signatures indicating completion of the forms. However, the completed 435.88 forms were provided, upon request, as included in record transmittal files. The unsigned forms had been captured in the shipment file for later review or validation, prior to submitting the file to records management. 


\section{ASSESSMENT RESULTS}

In accordance with MCP-4252 “INL Quality Assurance Oversight” Revision 3, objective evidence has been gathered to demonstrate effective implementation of the requirements listed in the checklists. Identified conditions/issues and observations/recommendations are noted in the completed checklists and are discussed, in this summary report below. Conditions constitute a failure, malfunction, deficiency, defective item, or non-conformance. A Condition Report (CO) is typically generated to assist management with tracking and completion of actions to resolve conditions adverse to quality.

One Condition was identified and communicated to the appropriate management personnel. The condition was corrected during the assessment. The condition was presented as follows:

\section{Conditions/Issues}

Condition: CO 2020-1769 - PDD-1078 “Waste Generator Services Technical Qualification Program" and MCP-17500 Requires:

If you are an individual who: Performs Nevada National Security Site (NNSS) waste certification; oversees the NNSS waste certification process; coordinates Idaho National Laboratory distribution of changes to NNSS requirements documents and forms; reviews completed documentation; conducts and controls assessments; verifies program implementation, training, and qualification; completes applicable shipment checklist; ensures adequate validation of analytical data; and reviews and approves waste characterization and shipping documentation; you must complete Job Code WGCONLCTOF, BEA NNSS Waste Certification Official.

One Alternate Waste Certification Official, who had signed as a WCO on reviewed shipment documents, did not have the WGCONLCTOF (WCO) job code assigned to his training plan, as required. It was, however, observed that the AWCO had completed all the required qualifications associated with the job code, and had been accepted through the NNSS approval process. The missing job code appears to have been an administrative oversight. During the assessment, the WGS Manager notified the Training Coordinator and the job code was added to the individual's training plan.

\section{Observations/Recommendations}

The following Observations/Recommendations are provided to management personnel as a result of this assessment.

Observation: SG 2020-0581 - PLN-4385 and PDD-17000 reference INL documents that are no longer current, such as LWP-4002, "Service Acquisitions” and PLN-522, "Quality Assurance Program Plan for the Waste Management/Waste Certification Program”. It is recommended that they be considered for revision. Also referenced in PDD-17000 is PLN-3318 that is an inactive document. 
Observation: SG 2020-0583 - PLN-17522 Rev. 0 does not include reference to a training/qualification plan. PLN-552 (PLN-17522's predecessor) called out PDD-1078 as the program description document for training and qualification. It is suggested that PLN-17522 include a reference to a qualification/training program.

Observation: SG 2020-0584 - Weekly WGS Reports contain INL Corrective Actions (CA) as documented in Labway. WGS personnel then track CAs to closure to assure any issue associated with waste streams are corrected. A review of WGS reports shows that CAs for all INL conditions; including for some nuclear facilities that may or may not be business sensitive are tracked. The suggestion is to work with the INL Issues Management Group to build a query tool for WGS to only track CAs that are pertinent to their mission. Also, this would reduce the burden of WGS personnel from screening and tracking all CAs.

Observation: SG 2020- 0585 - Some documents, included in shipping files, do not indicate they are under form control (i.e., Form No., revision date). As a suggestion, evaluate forms used in shipping packages to determine if the forms should be controlled using a form number and revision number.

Observation: SG 2020-0586 - INL Form 435.B04 'NNSS Driver Briefing” does not contain a field to link the completed form to a specific shipment.

Observation: SG 2020-0587 - DOE-STD-1090 has been revised in 2020. The standard states that this technical standard is not mandated for use at DOE sites. However, this assessment check list requires objective evidence that rigging devices, have a current load test that meets the DOE standard. It is suggested that waste management personnel coordinate with Hoisting and Rigging or Packaging and Transportation personnel to evaluate the program to latest revision of the DOE standard to assure any deltas are understood and addressed.

Observation: SG 2020-0588 - For Purchase Order (PO): 00129719, the original PO in Asset Suites did not have the correct address of the vendor as listed in the INL QSL. Revisions of the PO (1 and 2) did have the correct address of Clinton TN. This could be an error trap if Asset Suite has multiple addresses for QL1 suppliers.

Observation: CO 2020-1449 - LWP-13120 "Identifying and Controlling Items" has field changes initiated in June of 2017. LWP-9101 "INL Procedure Usage" states: "Generally, a revision "should be" initiated when published field changes are more than 6 months old". This condition was reported to Quality Program management as a management “expectation” rather than a requirement. Quality Program management initiated a condition report (CO 2020-1449) based on less than adequate timeliness in resolving a field change. At the time of this report the CO had been resolved by revising LWP-13120.

The completed documentation and processes observed during this assessment demonstrate that the overall BEA/Atkins Waste Generator Services (WGS) Waste Management Program is "effective." 


\section{ASSESSMENT TEAM}

\begin{tabular}{|l|l|l|}
\hline \multicolumn{1}{|c|}{ Team Member } & \multicolumn{1}{c|}{ Title / Position } & \multicolumn{1}{c|}{ Area(s) Evaluated } \\
\hline Jeff J. Fluckiger & Lead Assessor & Assessment process oversight \\
\hline D. Randall Allen & Assessor & $\begin{array}{l}\text { Radiological Waste Characterization } \\
\text { Chemical Characterization }\end{array}$ \\
& & $\begin{array}{l}\text { Waste Traceability } \\
\text { Waste Transportation }\end{array}$ \\
\hline Michael J. Kelso & & Quality Assurance \\
\hline
\end{tabular}

\section{APPENDICES}

Appendix A: Assessment Plan

Appendix B: Personnel Contacted/Observed Record During Assessment

Appendix C: Referenced Document List

Appendix D: NNSSWAC DOE/NV-325-16-00 Quality Assurance Checklist

Appendix E: NNSSWAC 325-16-00 Radiological Waste Characterization Checklist Appendix F: NNSSWAC DOE/NV--325-16-00 Chemical Characterization Checklist Appendix G: NNSSWAC 325-16-00 Waste Traceability Checklist Appendix H: NNSSWAC 325-16-00 Waste Transportation Assessment Checklist 


\section{Intentionally Blank}


Appendix A

Assessment Plan 


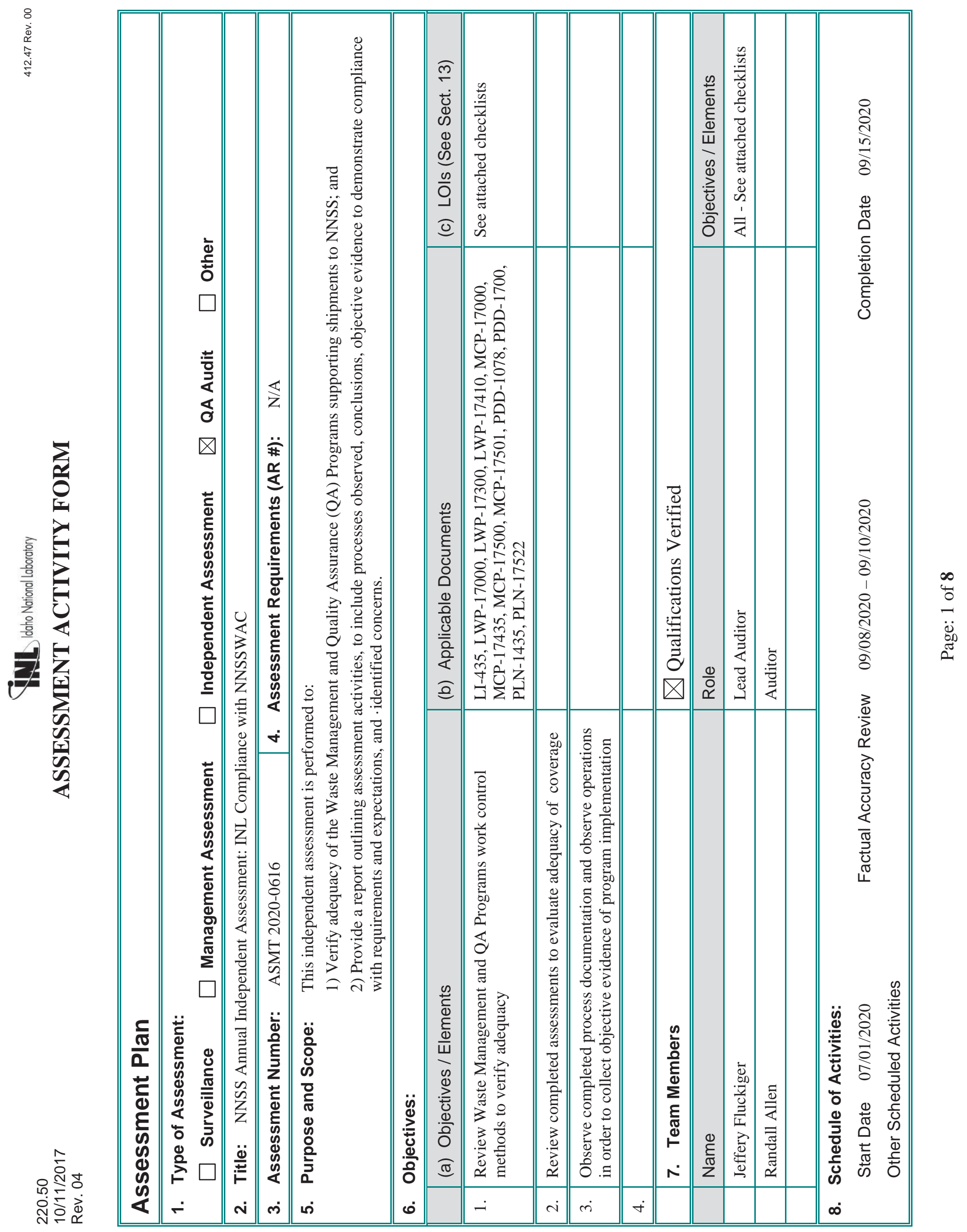




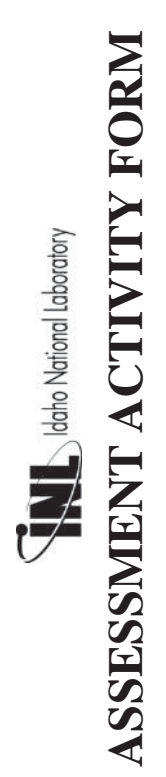

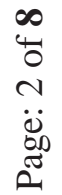

공ำ

ㅊํㅇ항 


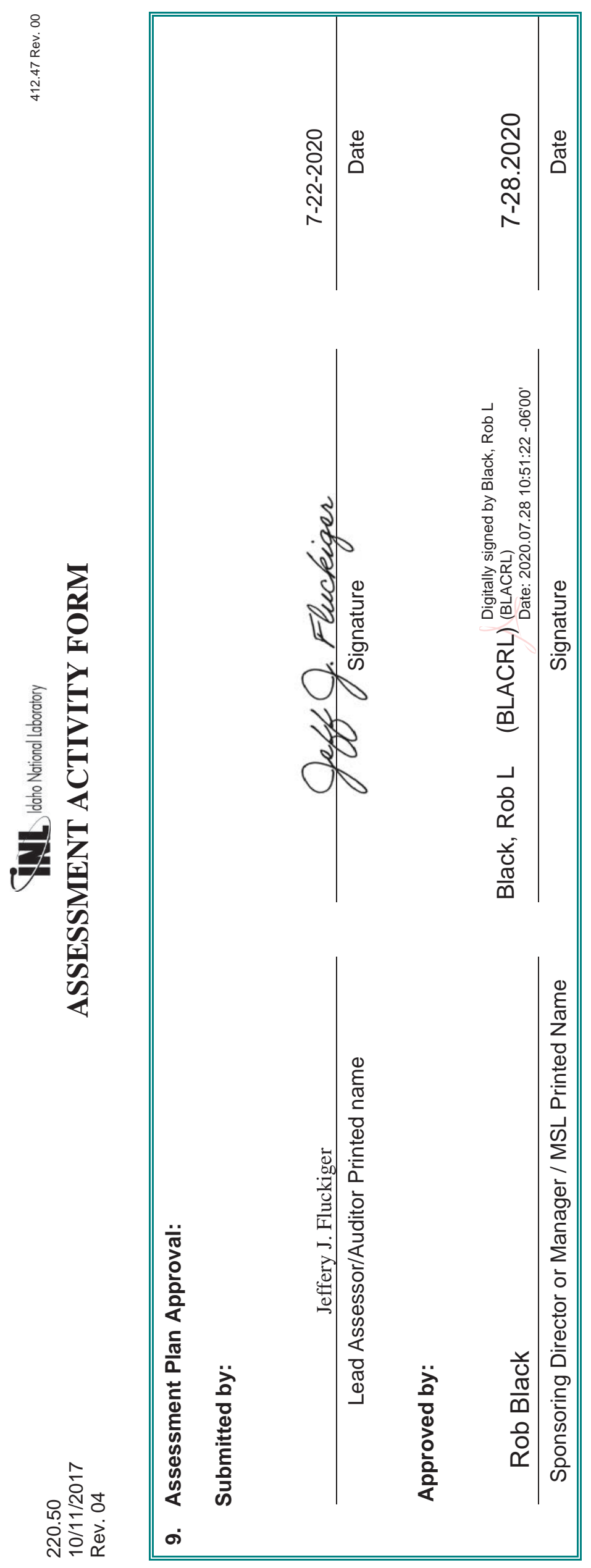

$\infty$
4
0
0
00
00
0
0 


\section{Intentionally Blank}




\section{Appendix B}

\section{Assessment Meeting/Contact Record}

\begin{tabular}{|l|l|}
\hline Name & Organization or Position \\
\hline Timothy Brown & Alternate Waste Certification Official \\
\hline Tyler L. Winder & Waste Generator Services - Project \& Program Support \\
\hline Larin D. Mortimer & Waste Generator Services - Project \& Program Support \\
\hline Dwayne B. Purser & ATR RadCon Operations \\
\hline Fred Hoffmeister & Truck Driver \\
\hline William J. Lambson & ATR RadCon Operations \\
\hline Stanley D. Zohner & Calibration Services \\
\hline Michael S. Stears & Calibration Services \\
\hline Kevin Ockerman & Instrumentation/Calibration Tech \\
\hline Nancy L Casebolt & Orano - Shipping \\
\hline Jeanie D. Hernandez & Packaging \& Transportation \\
\hline & \\
\hline
\end{tabular}




\section{Intentionally Blank}




\title{
Appendix C
}

\section{Waste Management Program Procedures and Forms Reviewed During the Assessment}

\author{
Work Control Documents \\ GDE-17233 “Waste Container Labeling” \\ LI-435 "Waste Management Routine Field Activities” \\ LWP-1202 “Records Management”, \\ LWP-2502 “Acquisition and Storage of Hazardous Materials Packaging” \\ LWP-4506 “Acceptance of Procured Items and Services” \\ LWP-10200 “Engineering Calculations and Analysis Report” \\ LWP-13120 "Identifying and Controlling Items" \\ LWP-15017 "Radiological Release Surveys” \\ LWP-15026 “BEA Methodologies for Characterization of Radioactive Material” \\ LWP-17000 “Waste Management” \\ MCP-139 "Radiological Surveys” \\ MCP-8523 “Managing Hazardous and Non-hazardous Samples” \\ MCP-9810 “Shipment and Receipt of Hazardous Materials” \\ MCP-9811 “Selection and Acquisition of Hazardous Material Packaging” \\ MCP-17000 “Waste Generator Services Waste Management” \\ MCP-17435 “Sorbent Selection and Use” \\ MCP-17500 "Waste Generator Services Certification of Waste Shipments to the Nevada National Security \\ Site” \\ PDD-1078 “Waste Generator Services Technical Qualification Program” \\ PDD-13000 “Quality Assurance Program Description” \\ PLN-4385 “IT Asset Maintenance Plan for the Integrated Waste Tracking System (IWTS)”
}


PLN-8510 "Planning and Management of Environmental Monitoring Sampling Activities"

PLN-17522 “Quality Assurance Program Plan for Low-Level, Mixed Low-Level and Hazardous Waste Management/Waste Certification”

\section{Process Forms}

435.B01, “NNSS Container fissile Material Limit Evaluation”

435.B04 "NNSS Driver Briefing”

435.39 "Waste Determination and Disposition Form” (WDDF)

435.42 "Radioactive Waste Inventory Sheets”

435.88 "NNSS Waste Profile Checklist”

$435.89 \quad$ "NNSS Shipment Checklist”

$441.45 \quad$ "Radiological Survey Report”

435.46 "Monthly Inspection Checklist”

435.93 "NNSS Waste Certification Official Shipment Checklist"

435.99 “Absorbent Determination Form”

460.13 "DOT Excepted and IP-1 Design Criteria Checklist”

953 “Analytical Lab Process Worksheet” 
Appendix D

NNSSWAC DOE/NV-325-16-00 Quality Assurance Checklist 


\section{Intentionally Blank}



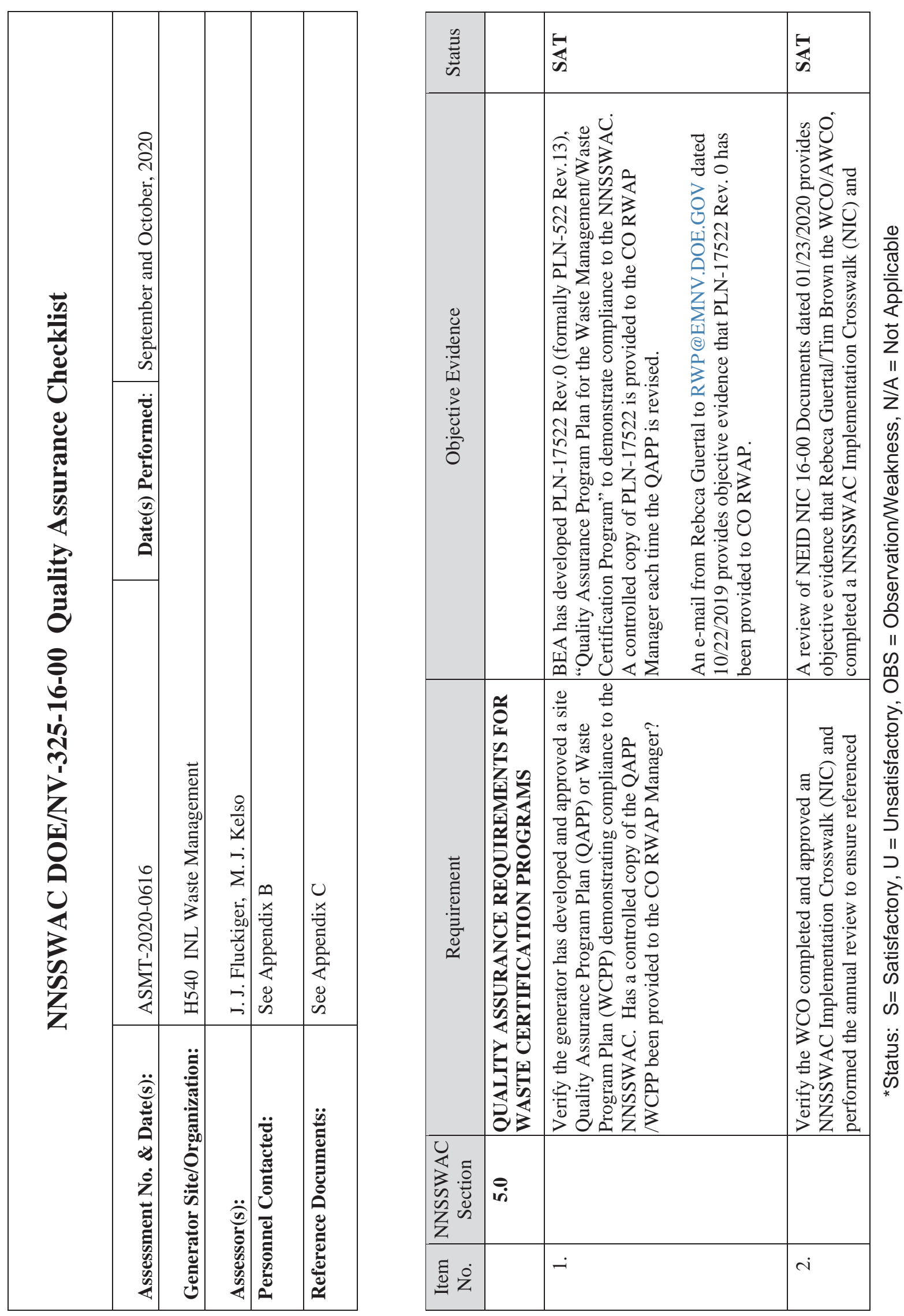


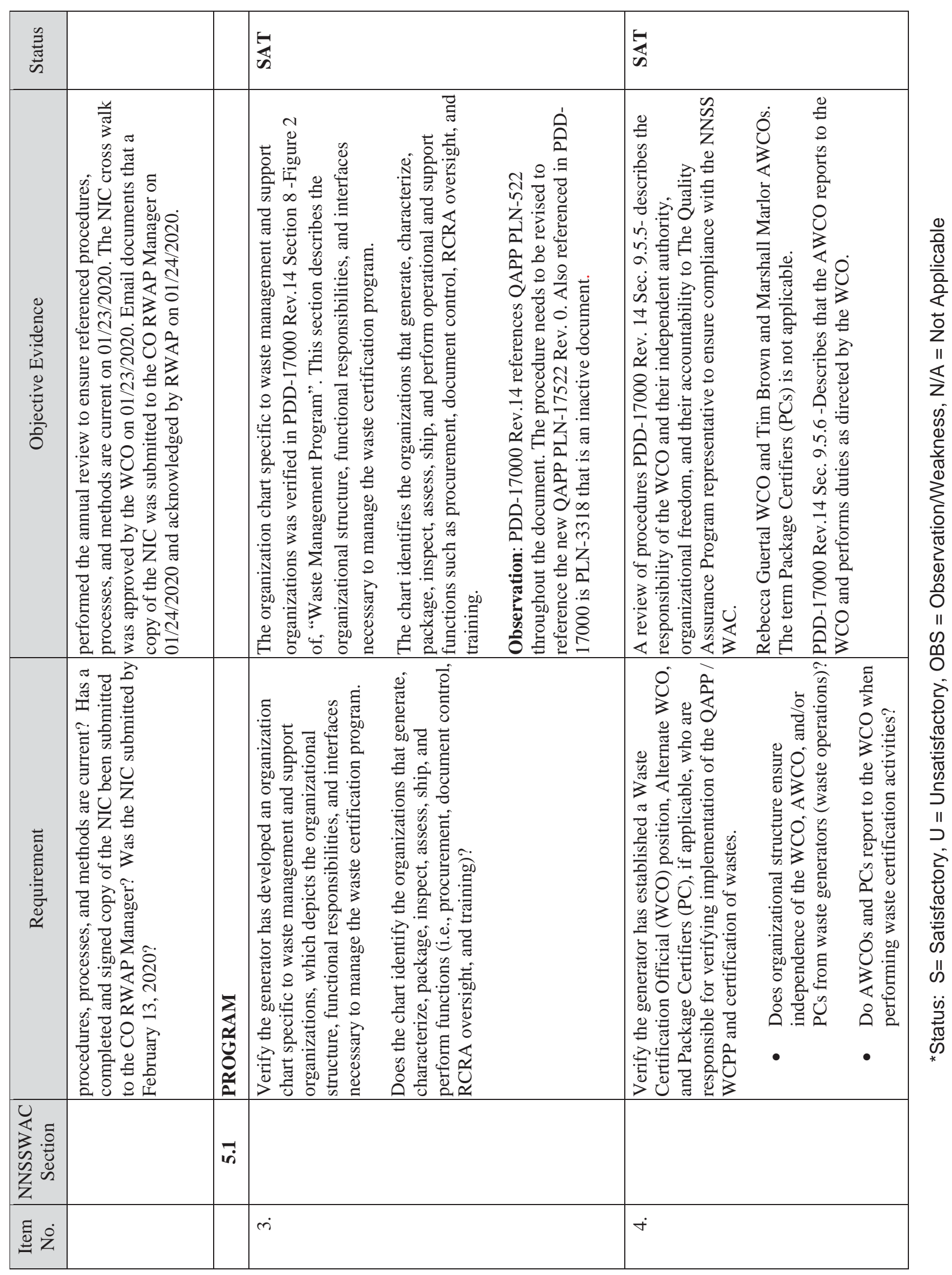




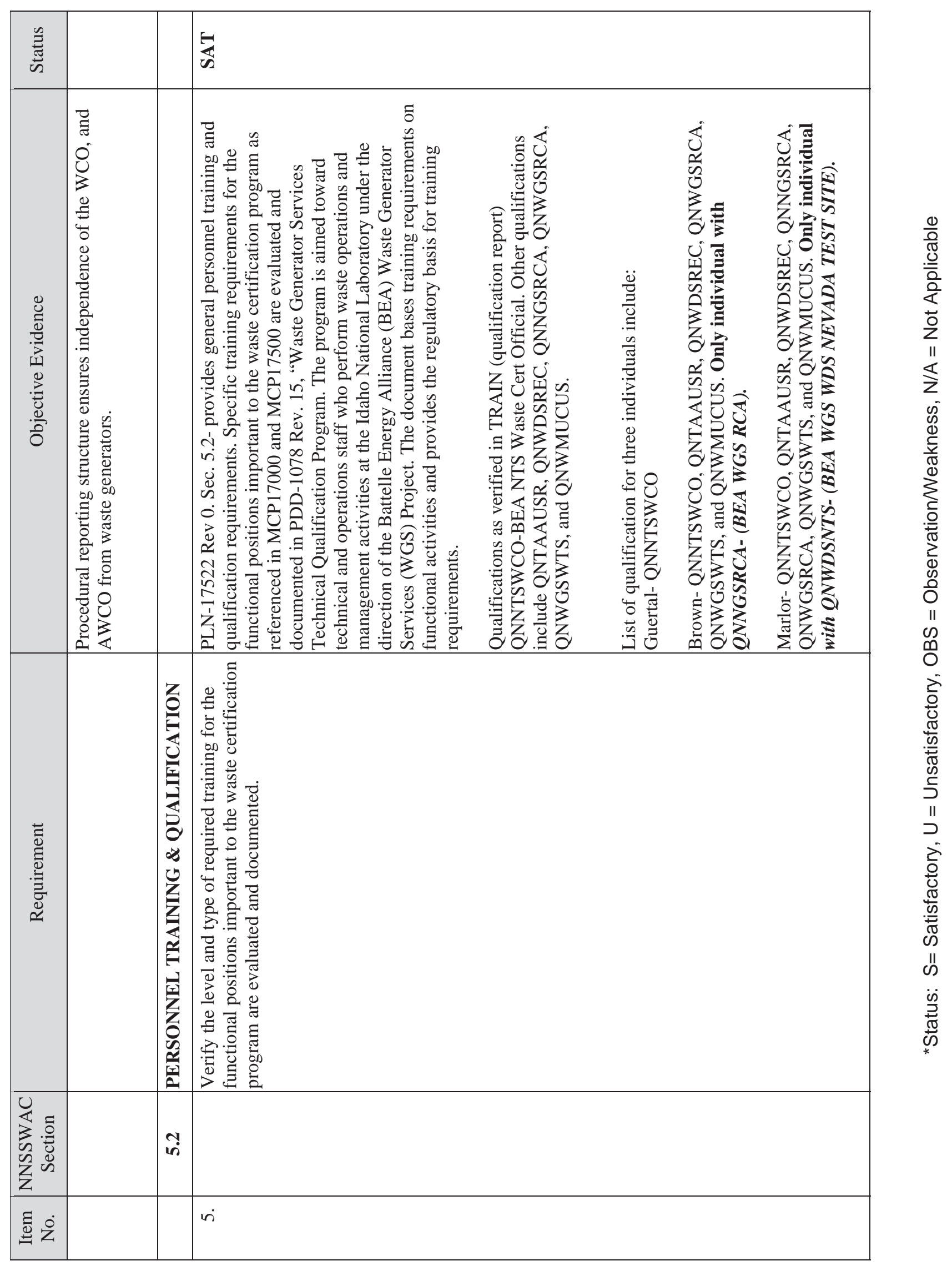




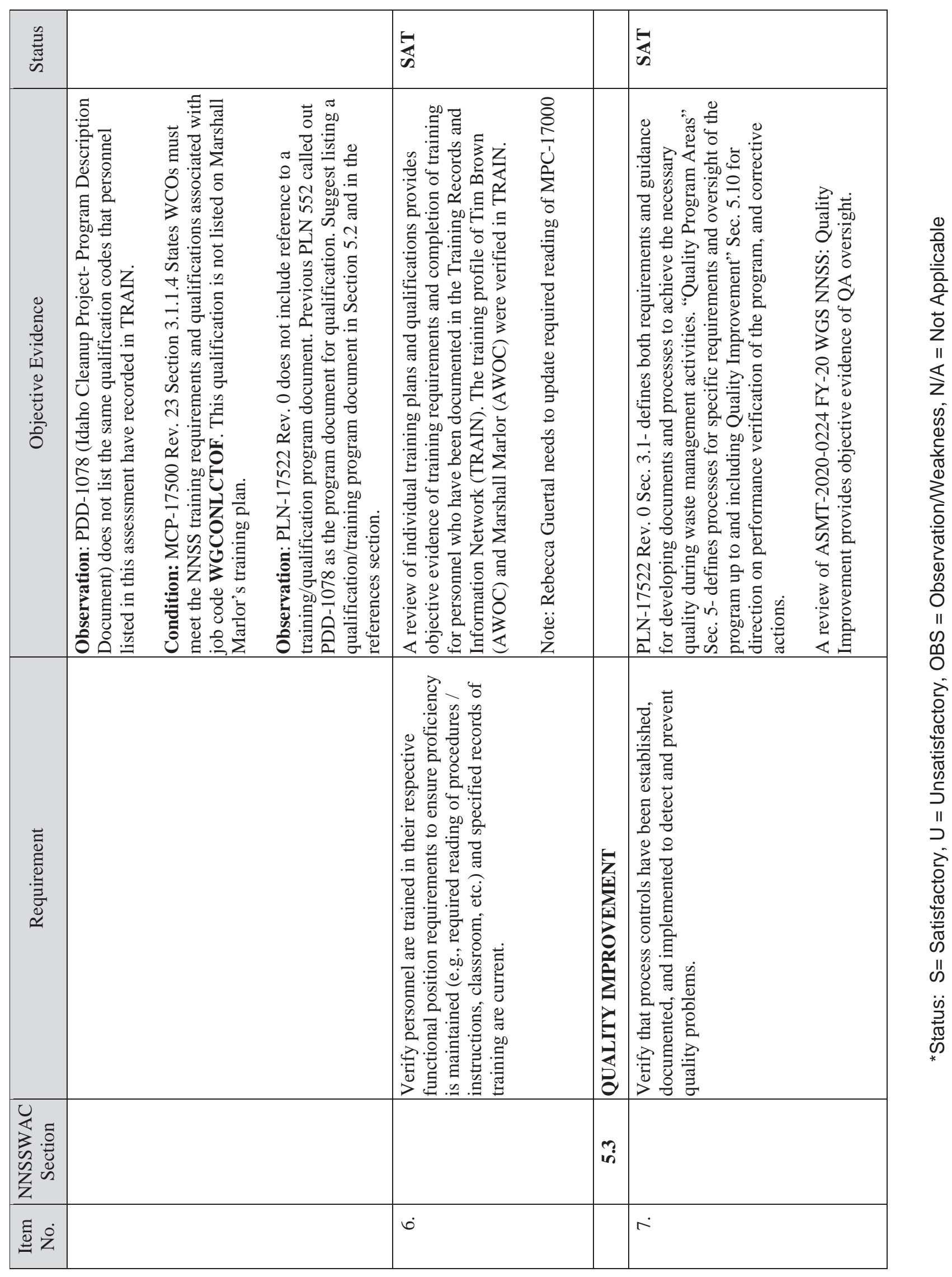




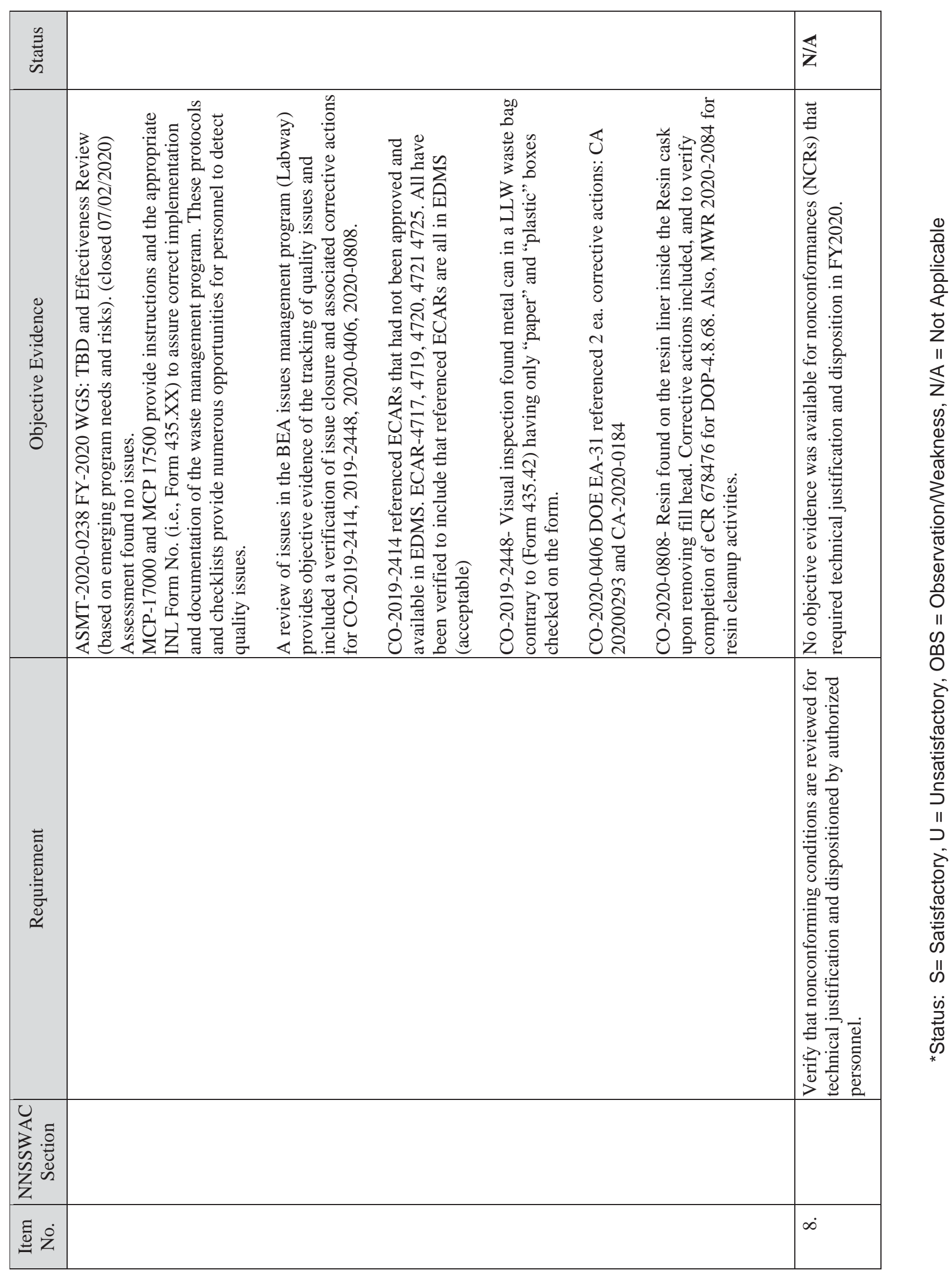




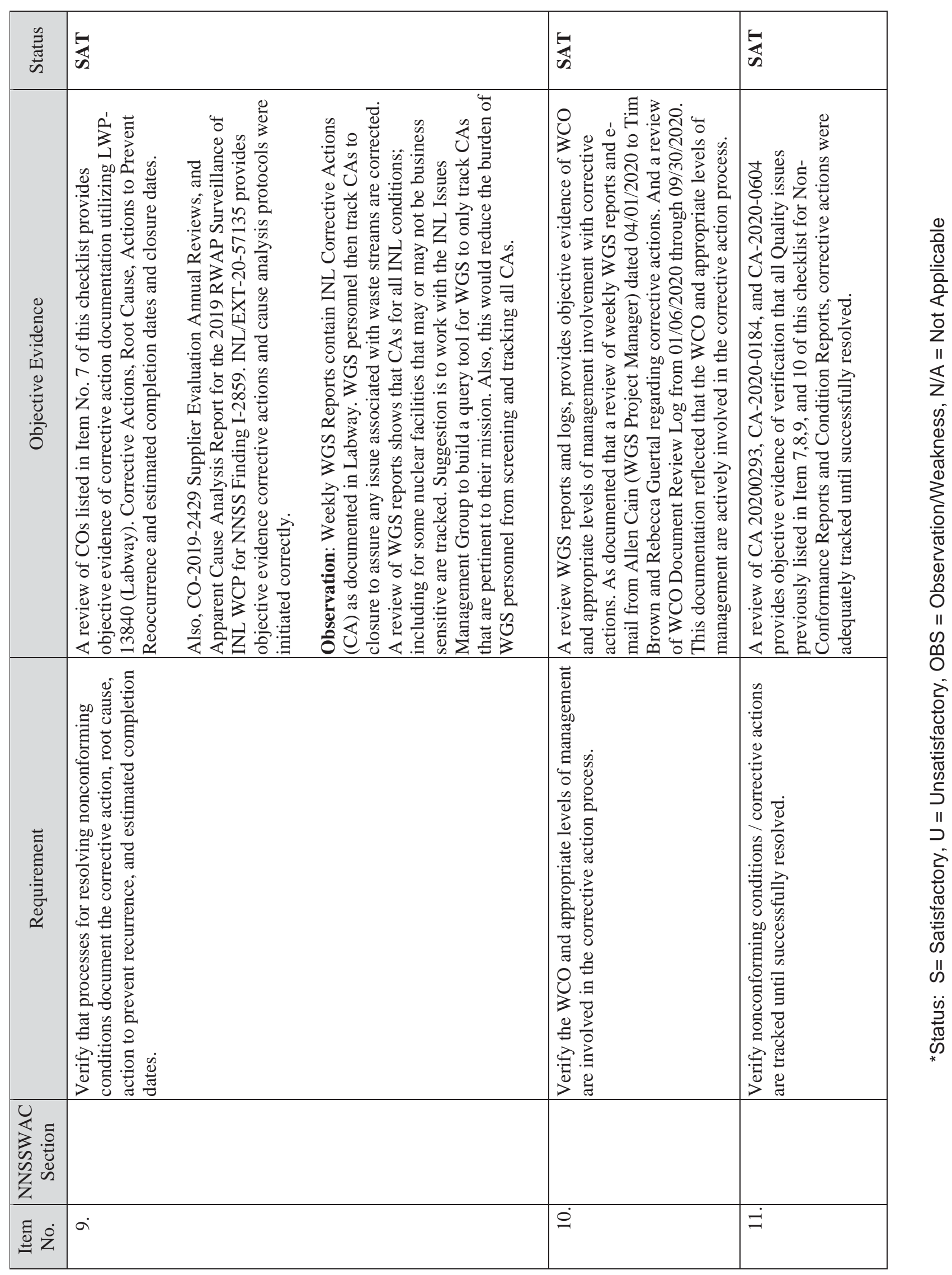




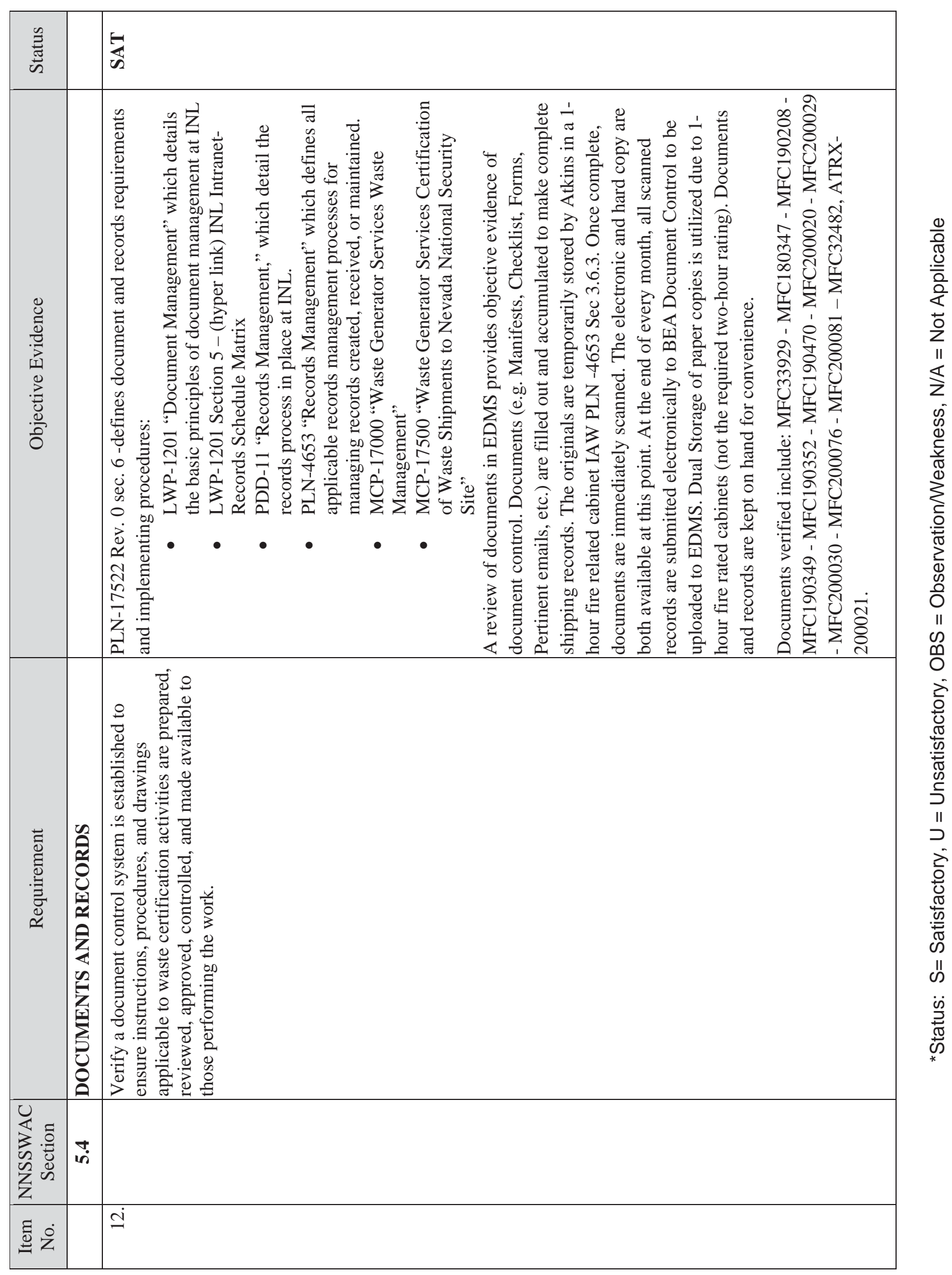




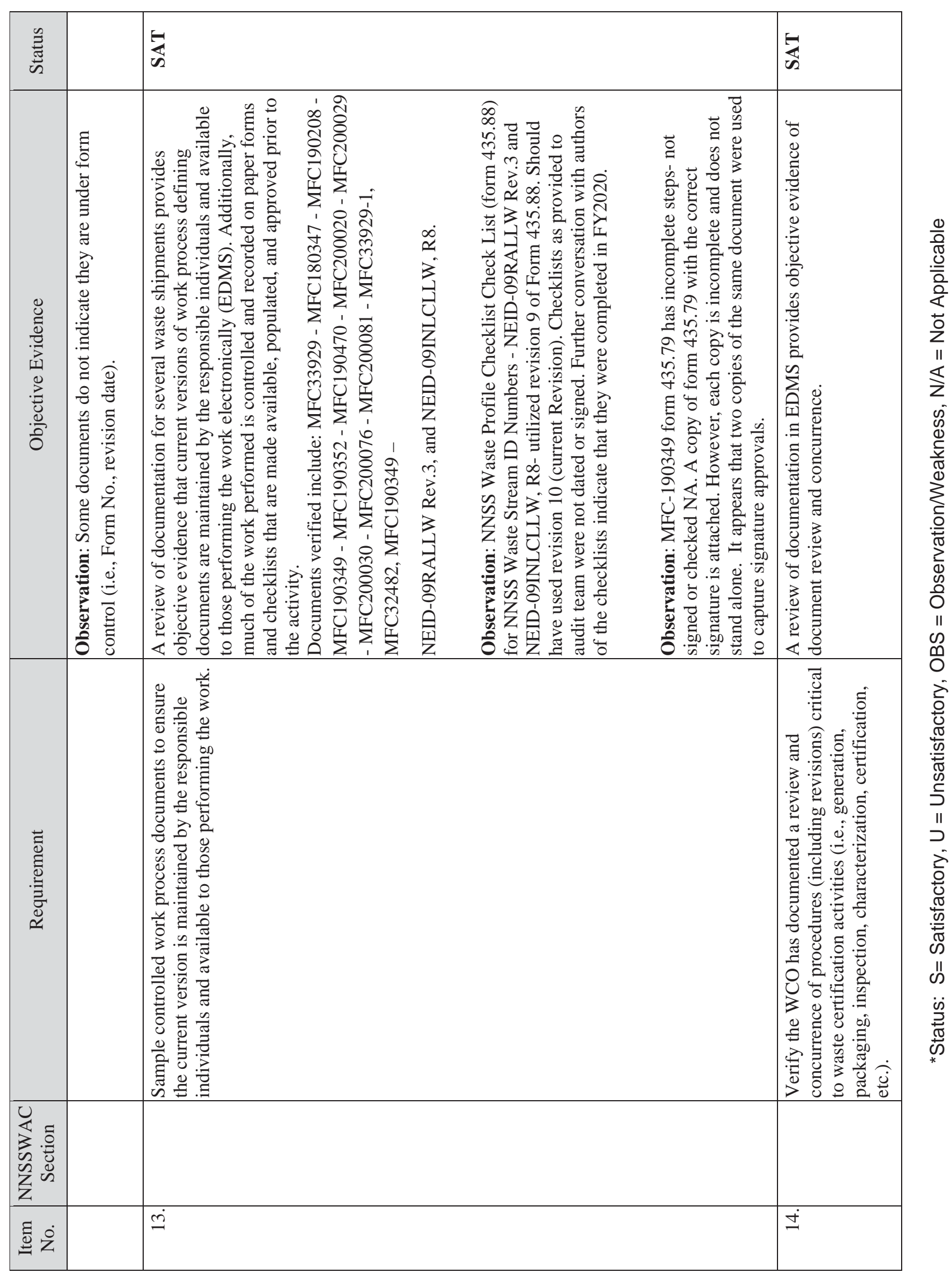




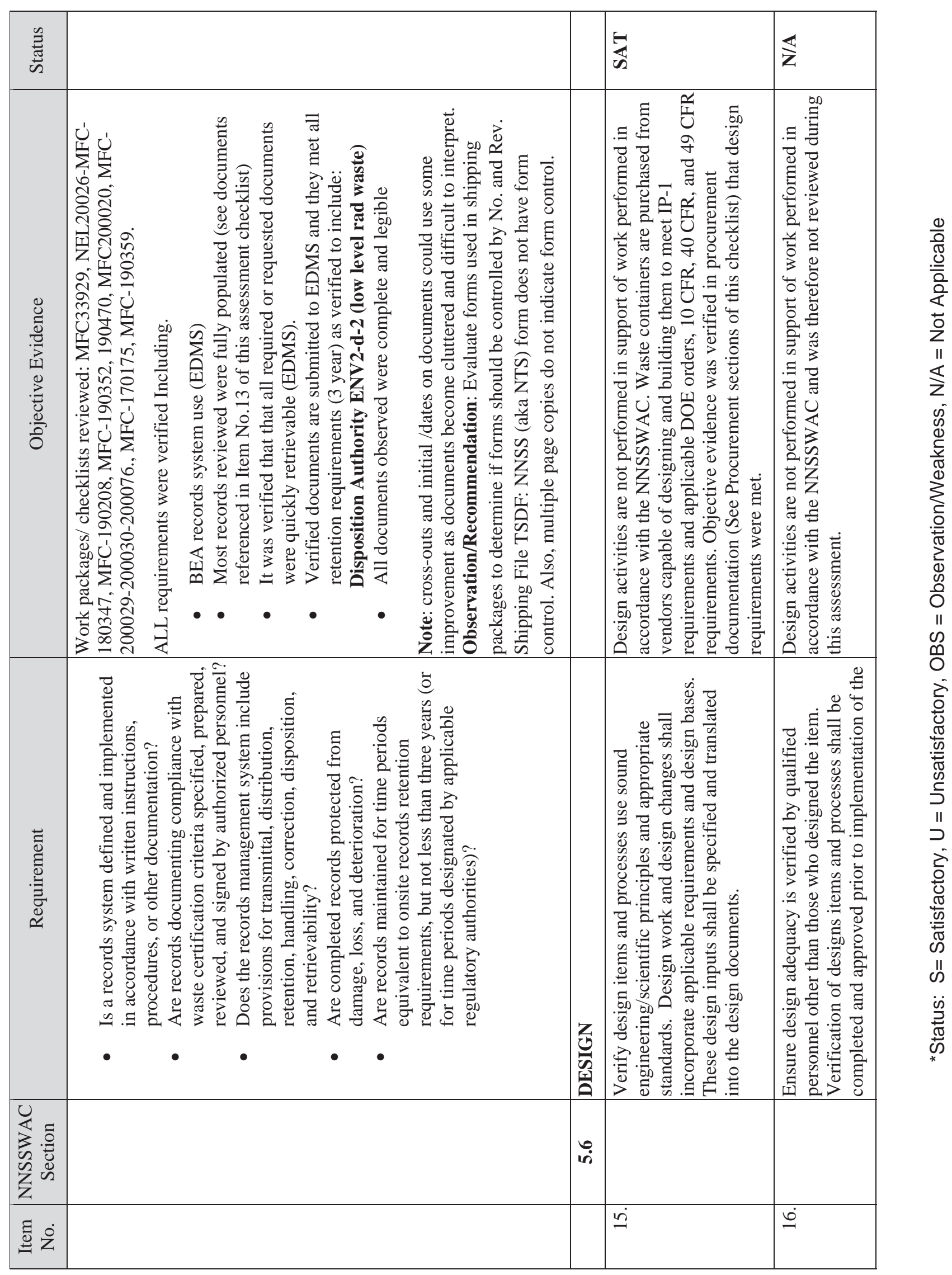




\begin{tabular}{|c|c|c|c|c|c|c|}
\hline 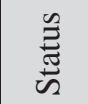 & & 离 & 妾 & 离 & & \\
\hline 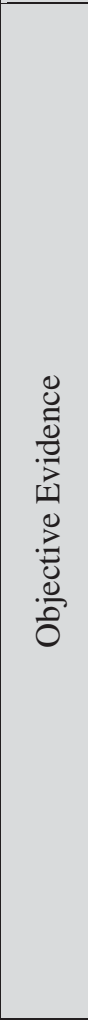 & & 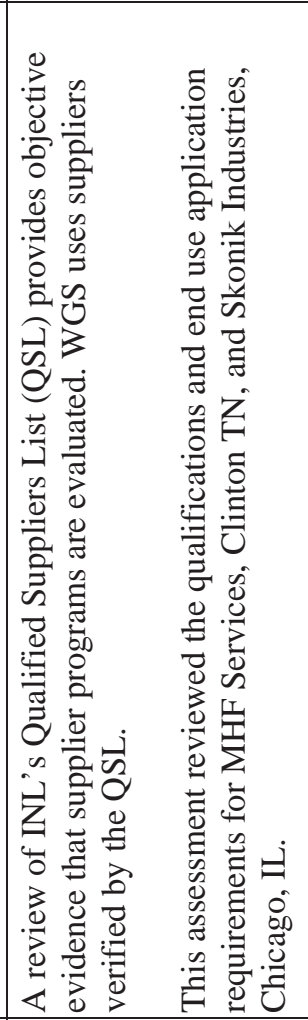 & 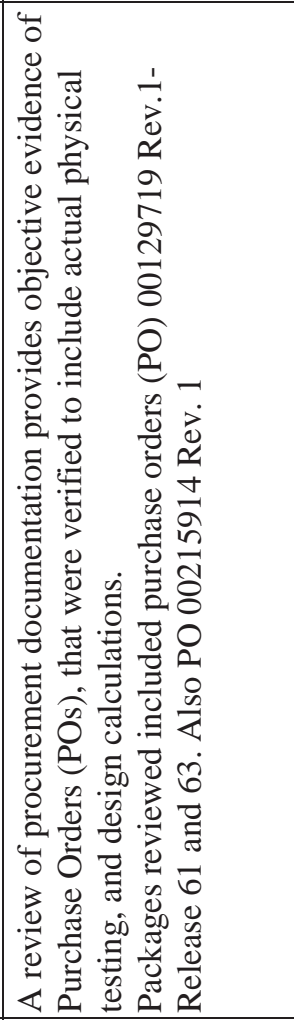 & 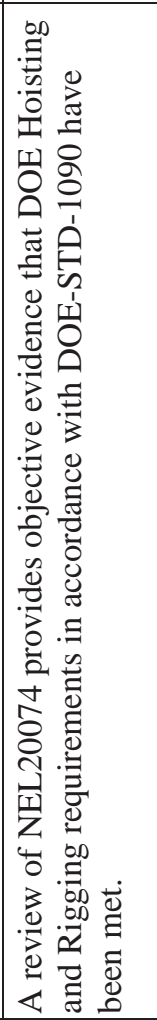 & 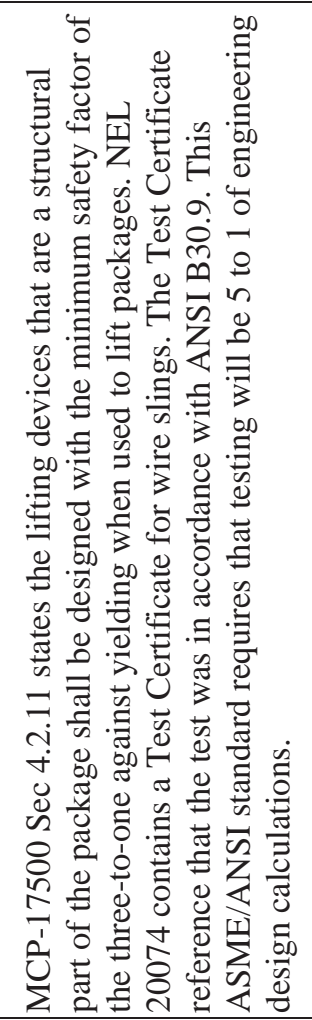 & 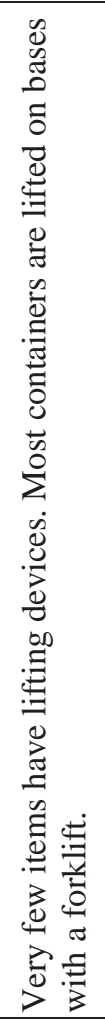 \\
\hline 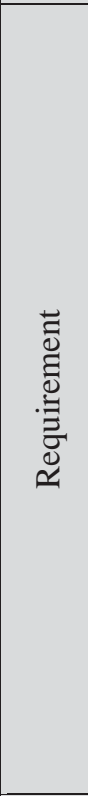 & 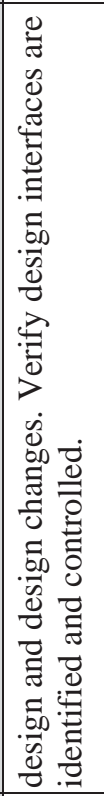 & 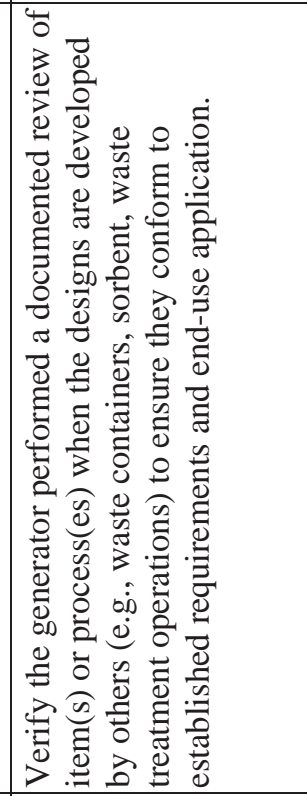 & 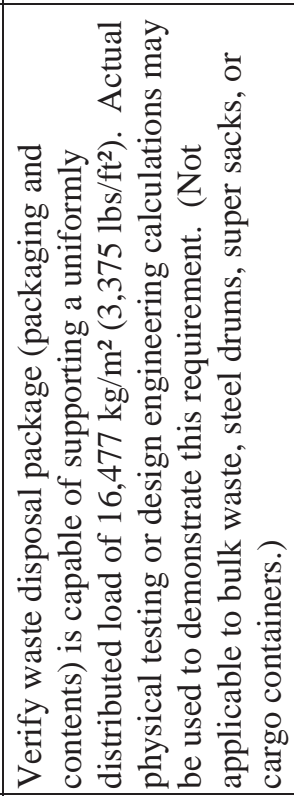 & 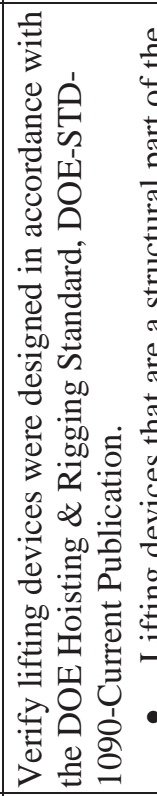 & 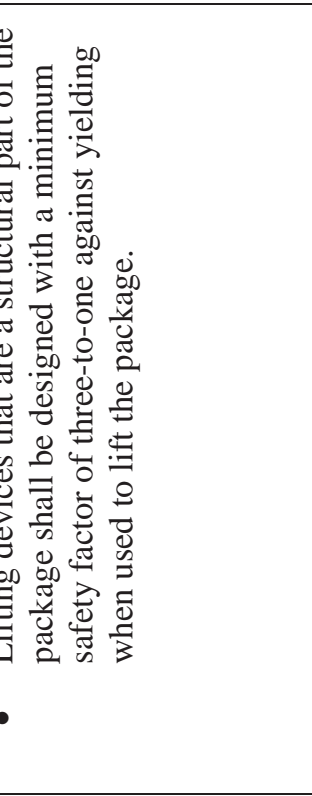 & \\
\hline 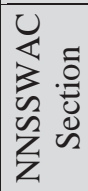 & & & |r & $\underset{\sim}{\tilde{n}}$ & & \\
\hline 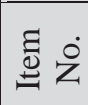 & & $\ddot{-}$ & $\stackrel{\infty}{\rightarrow}$ & $\dot{\sigma}$ & & \\
\hline
\end{tabular}




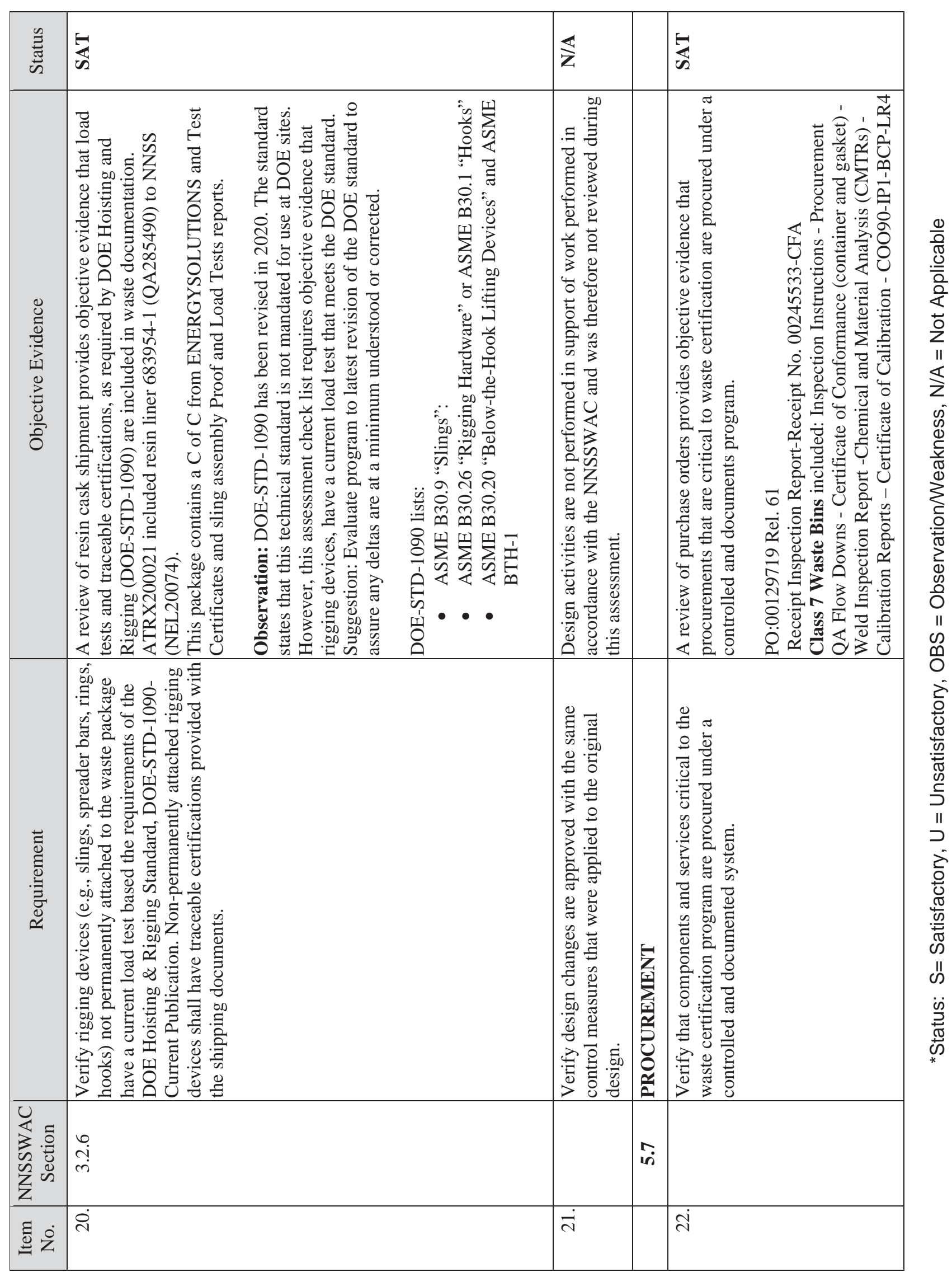




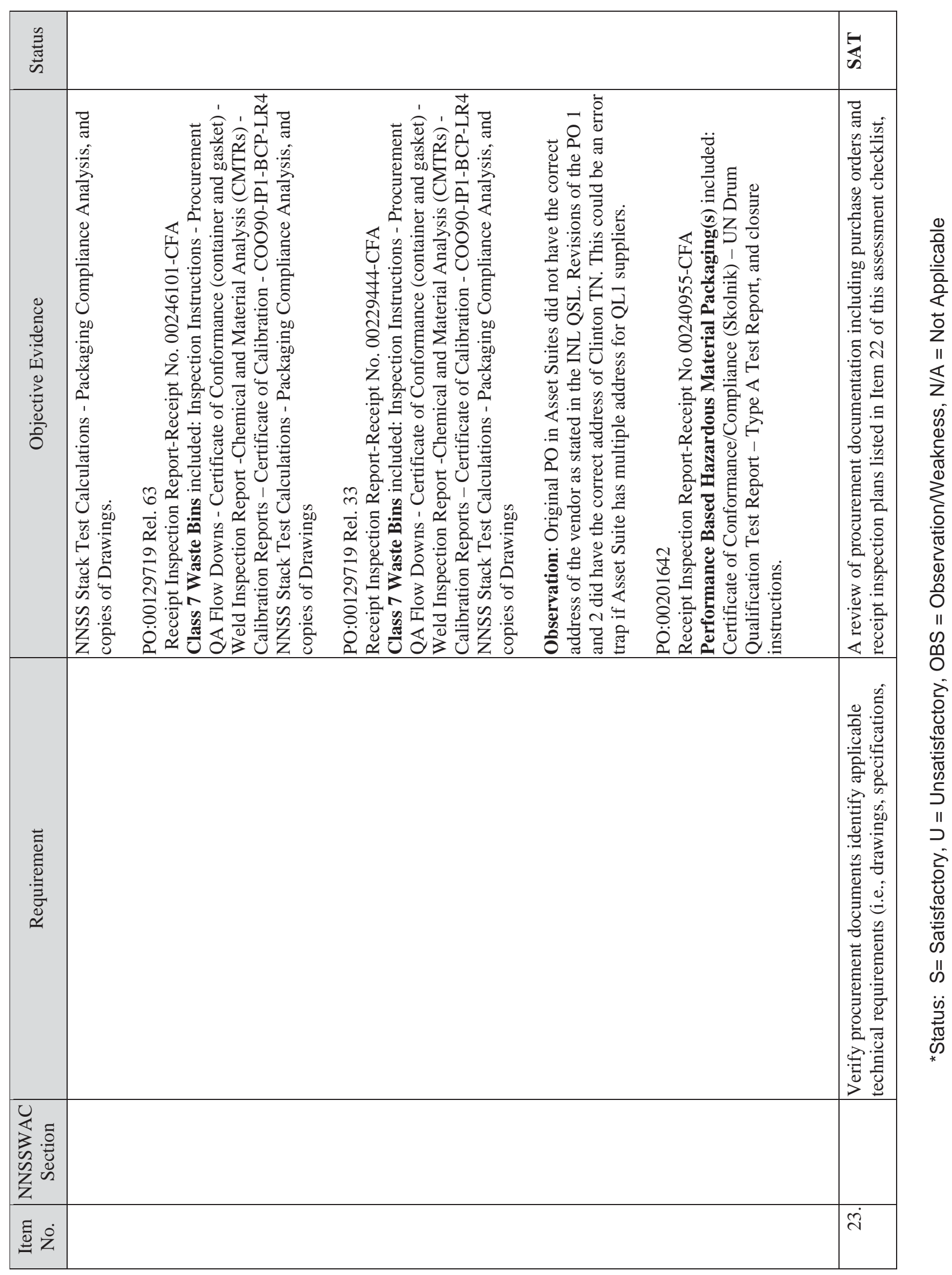




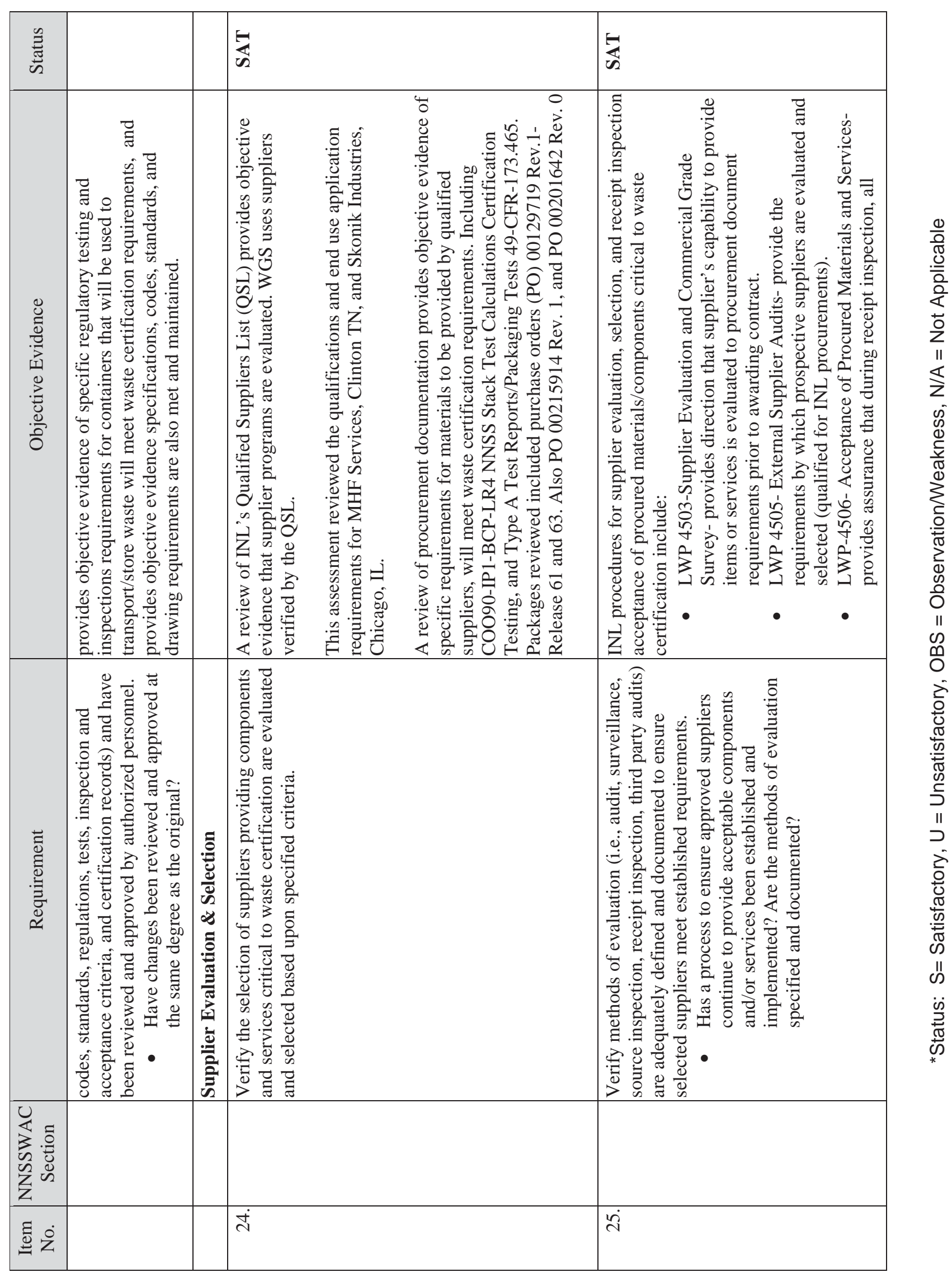




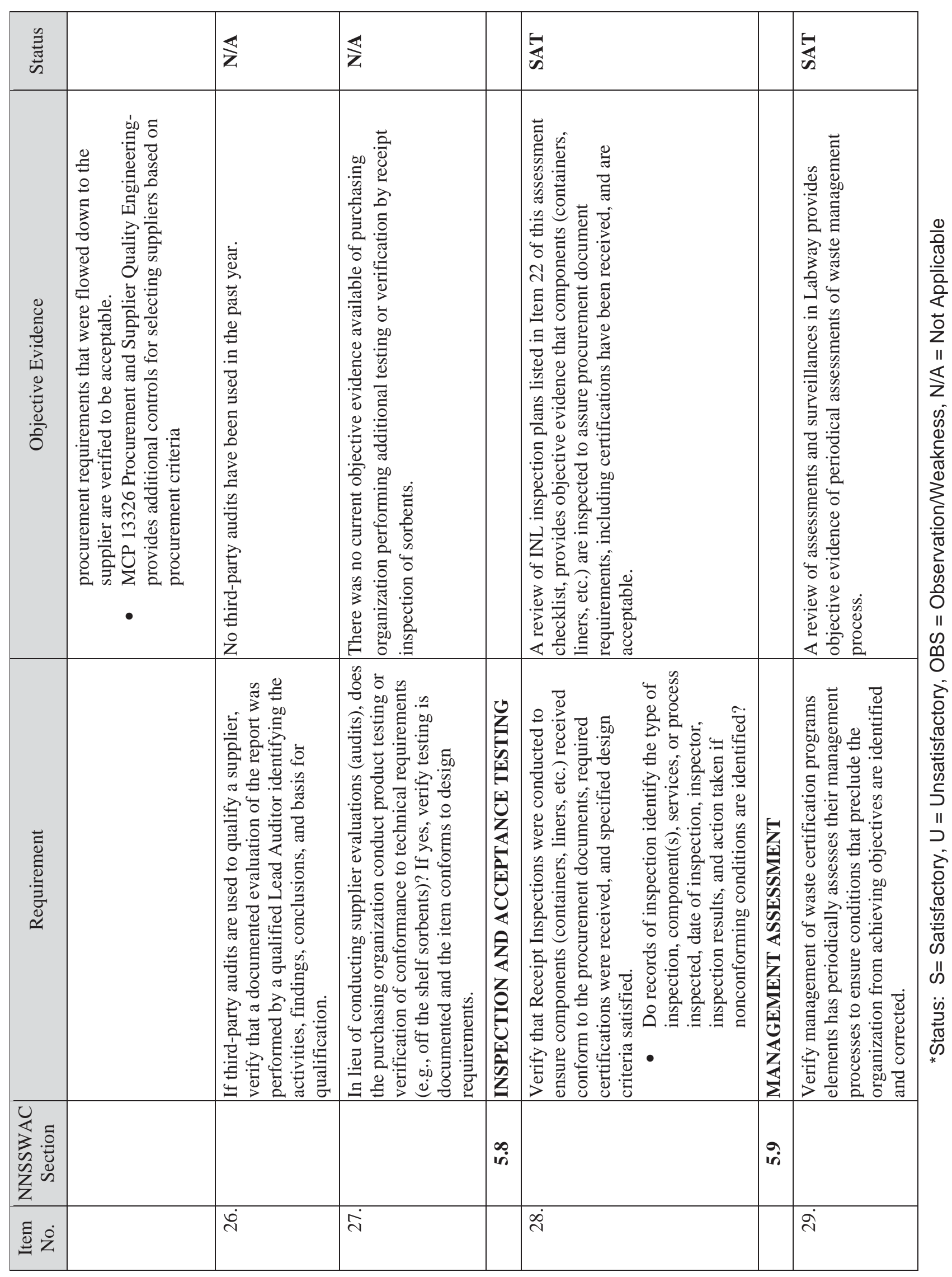




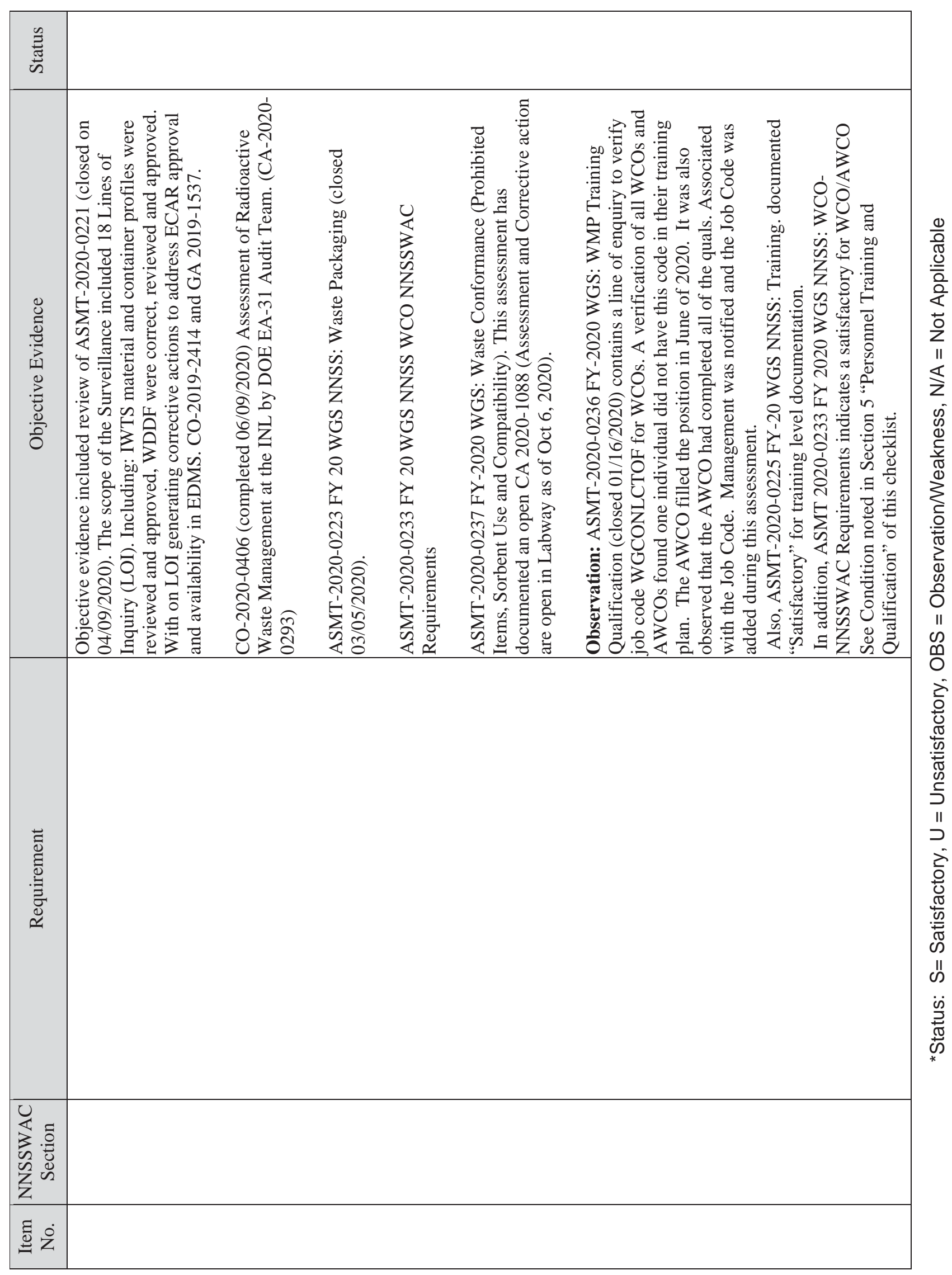




\begin{tabular}{|c|c|c|c|c|c|c|c|c|}
\hline 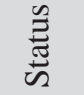 & & 隹 & & 倠 & & & & 㧫 \\
\hline 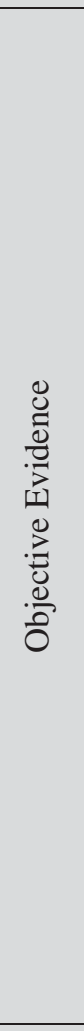 & 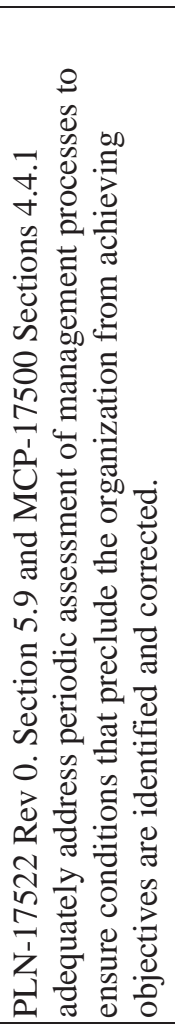 & 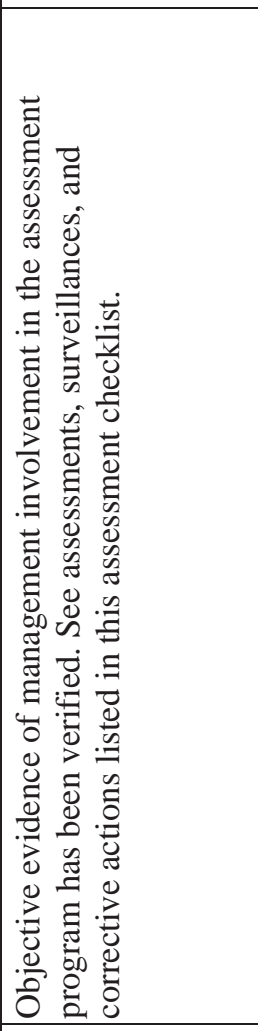 & & 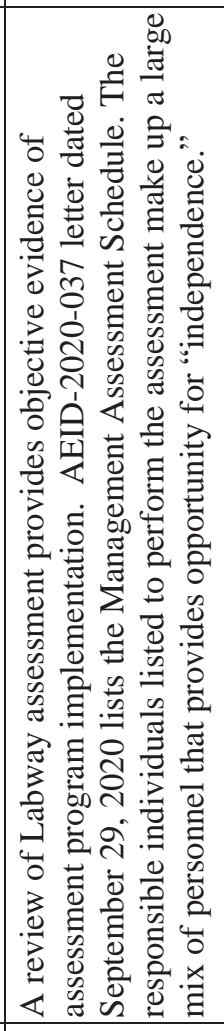 & 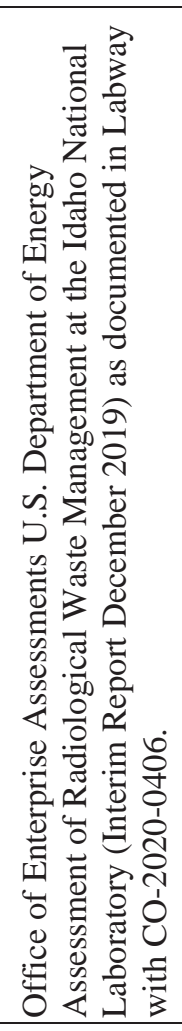 & 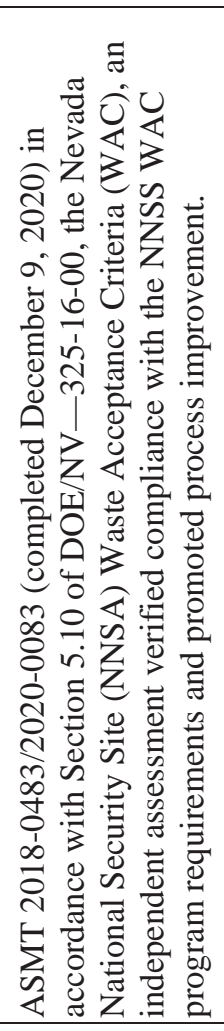 & 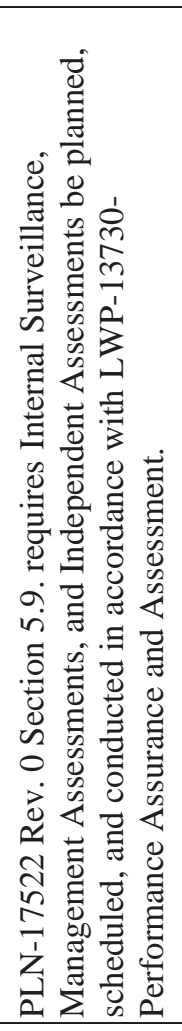 & 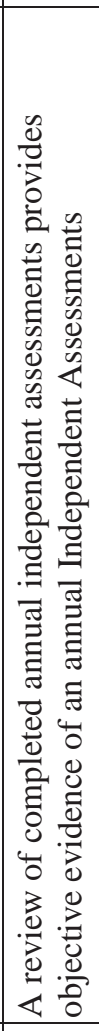 \\
\hline 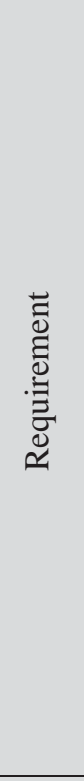 & & 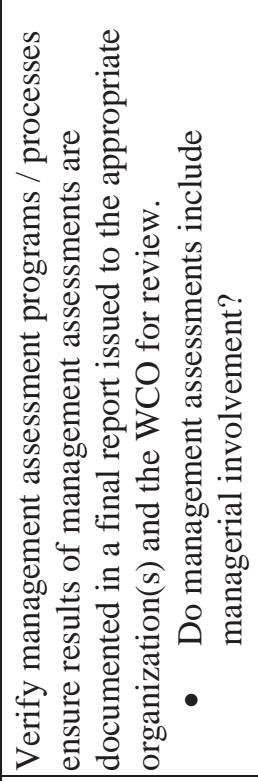 & 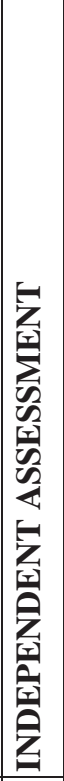 & 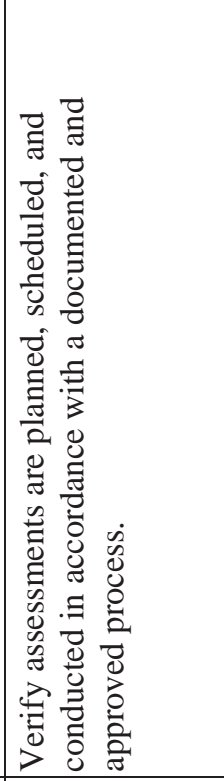 & & & & 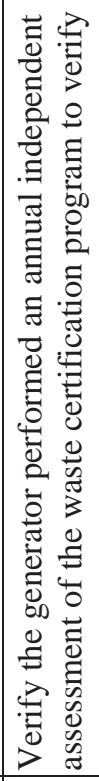 \\
\hline 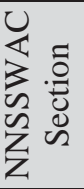 & & & $\begin{array}{l}0 \\
\text { in } \\
\text { is }\end{array}$ & & & & & \\
\hline E் & & ஜे & & $\vec{m}$ & & & & ஸें \\
\hline
\end{tabular}




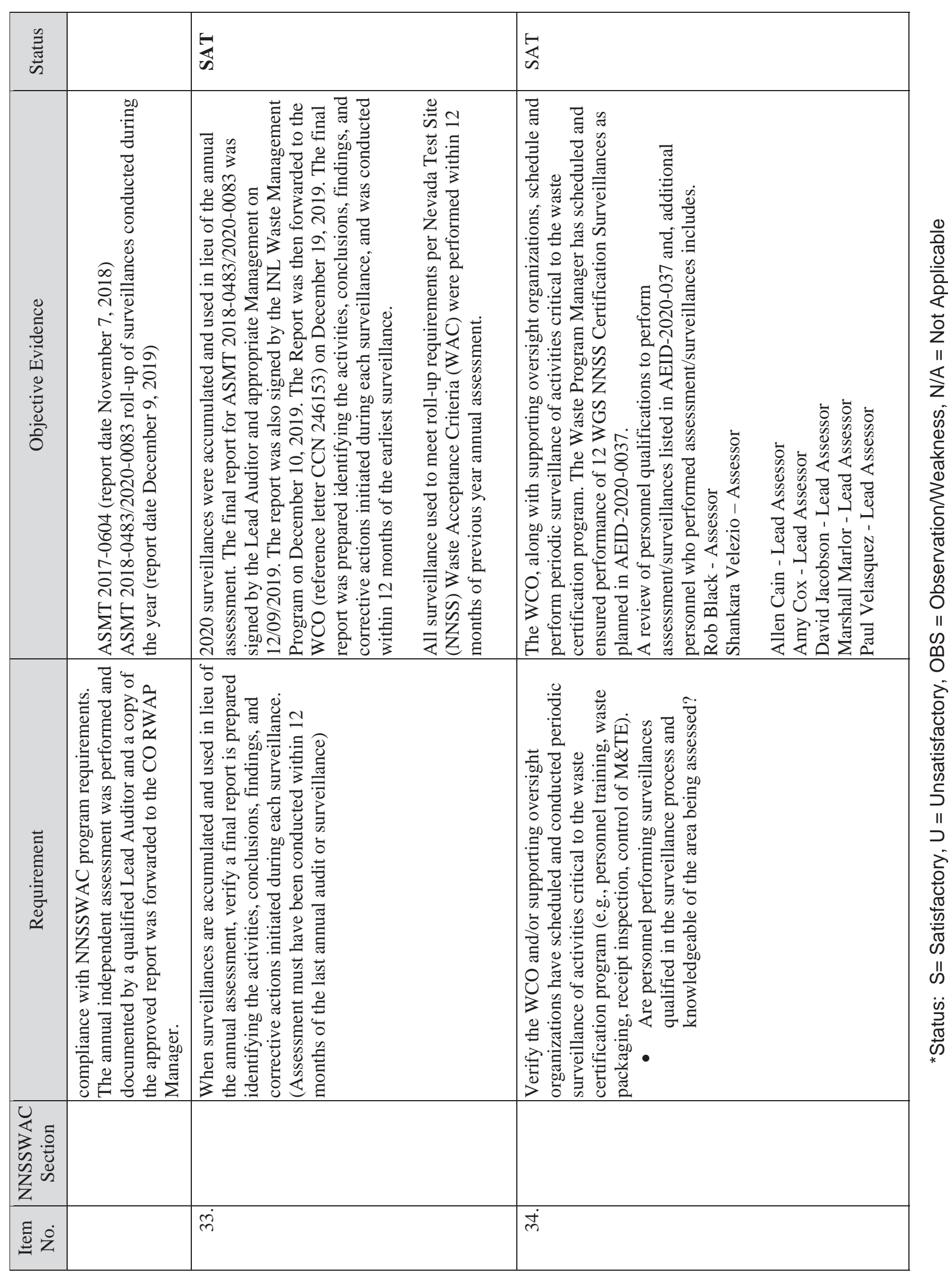




\begin{tabular}{|c|c|c|c|c|}
\hline 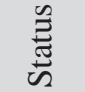 & & $\frac{5}{\omega}$ & $\frac{5}{6}$ & 皆 \\
\hline 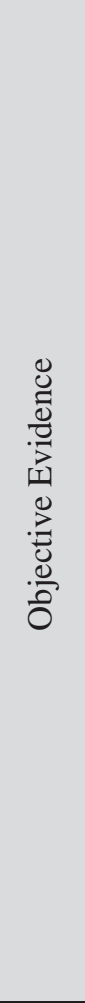 & 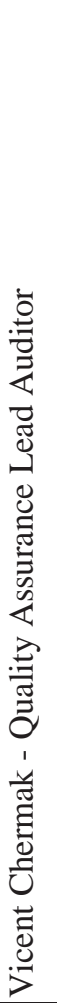 & 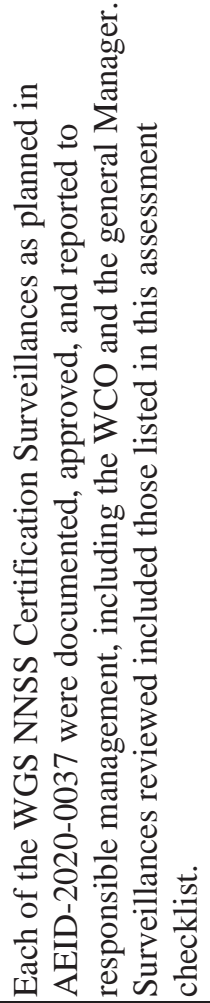 & 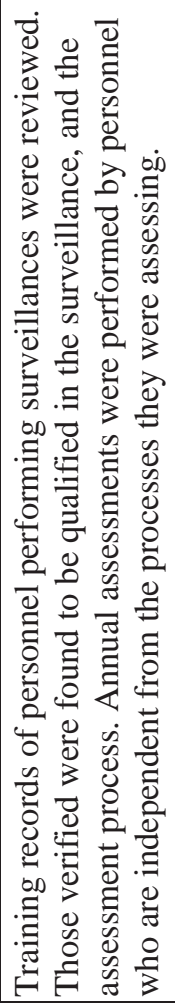 & 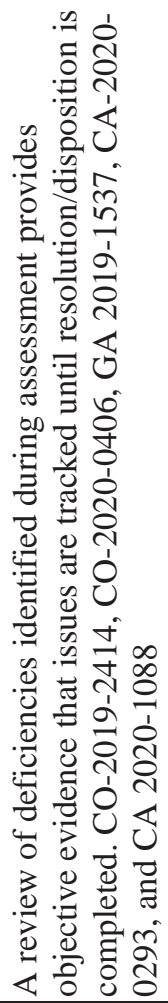 \\
\hline 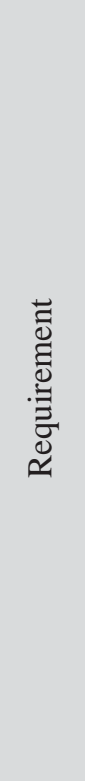 & & 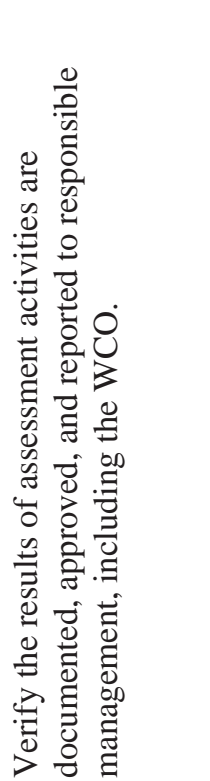 & 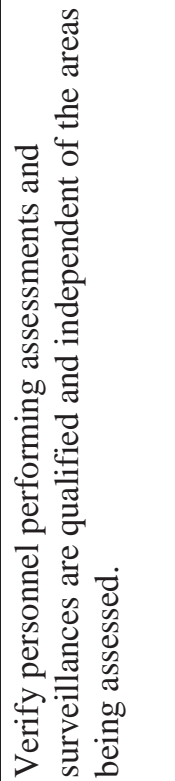 & 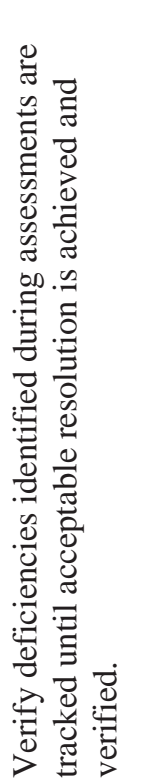 \\
\hline 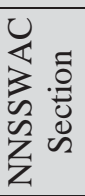 & & & & \\
\hline 吾 & & ம் & $\dot{\varphi}$ & $\hat{m}$ \\
\hline
\end{tabular}

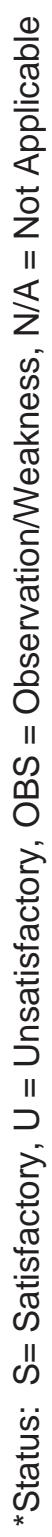




\section{Intentionally Blank}


Appendix E

\author{
NNSSWAC 325-16-00 \\ Radiological Waste Characterization Checklist
}




\section{Intentionally Blank}



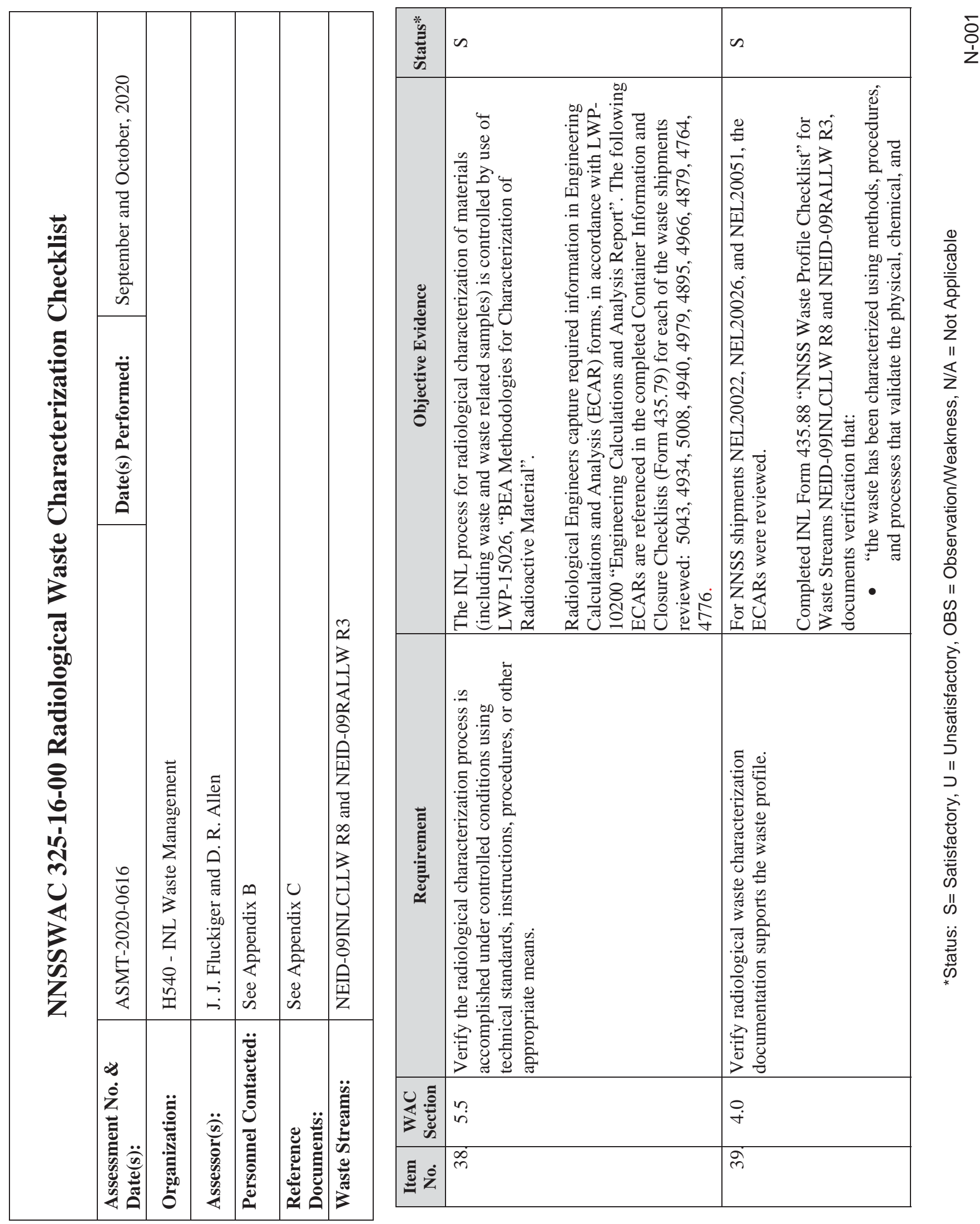


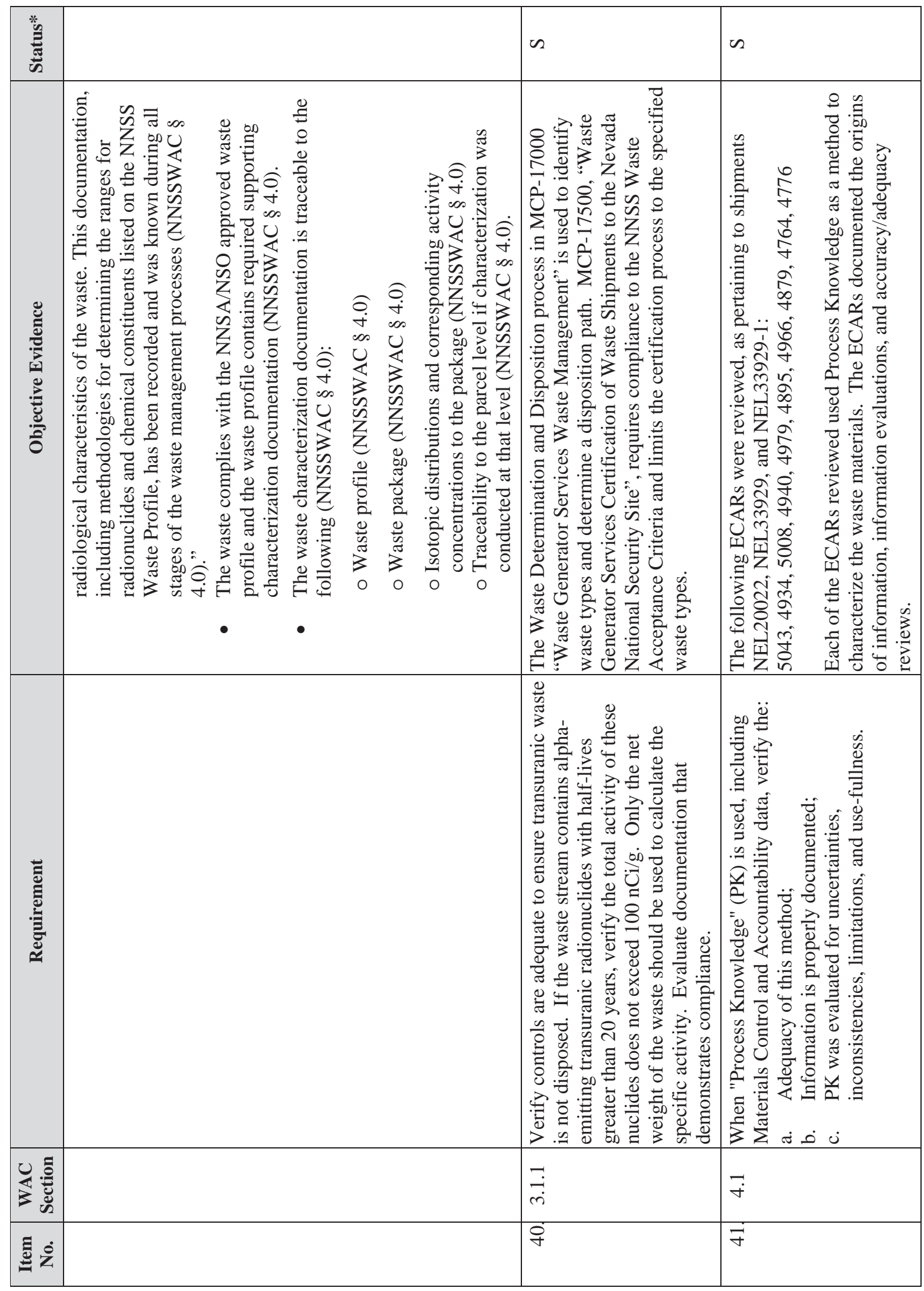

홀

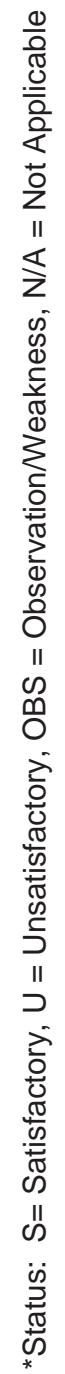




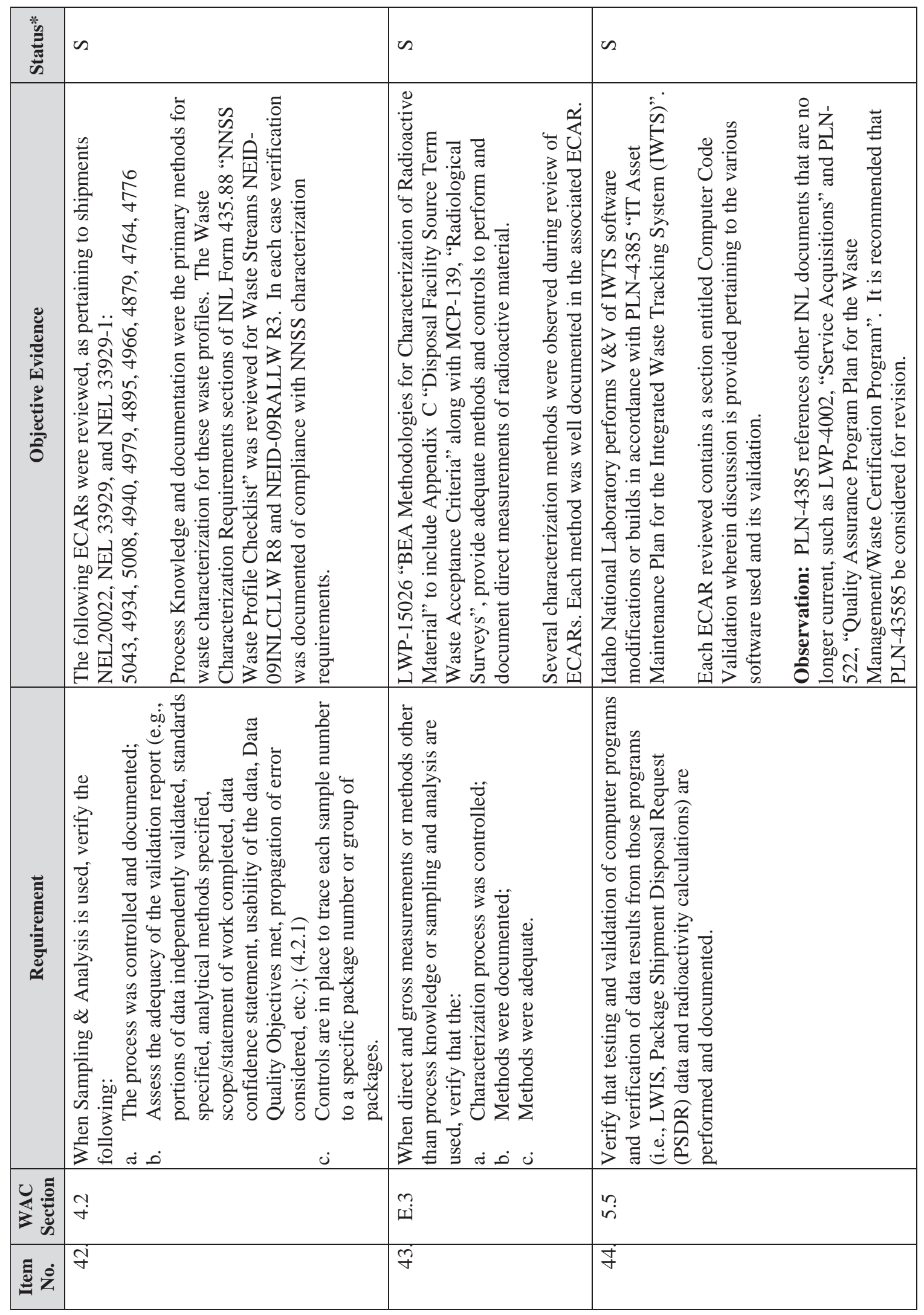

홀 


\begin{tabular}{|c|c|c|c|c|c|}
\hline 兽 & $n$ & $n$ & & $n$ & $n$ \\
\hline 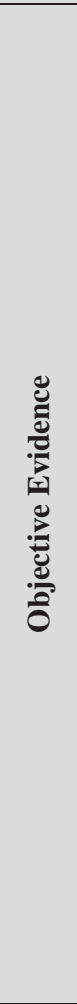 & 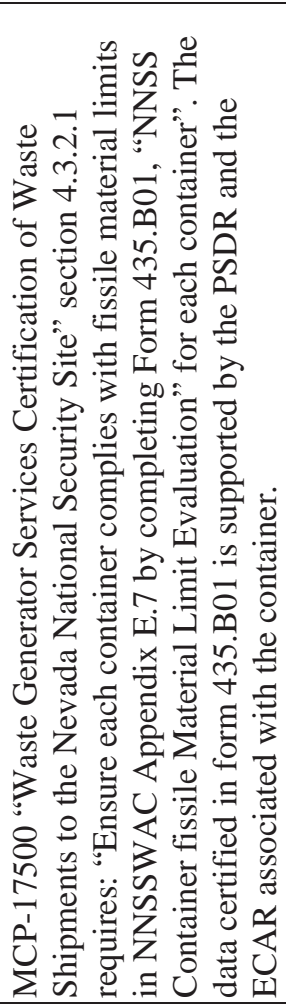 & 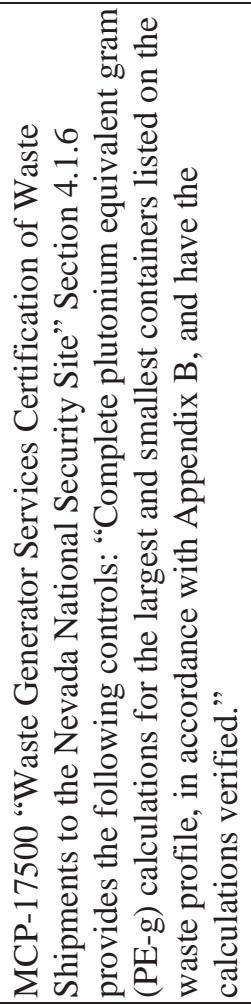 & 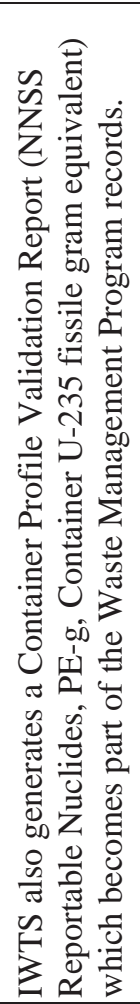 & 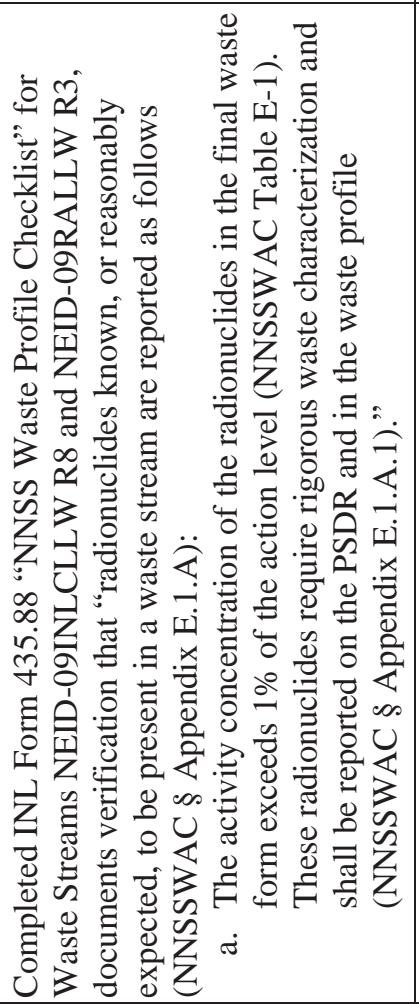 & 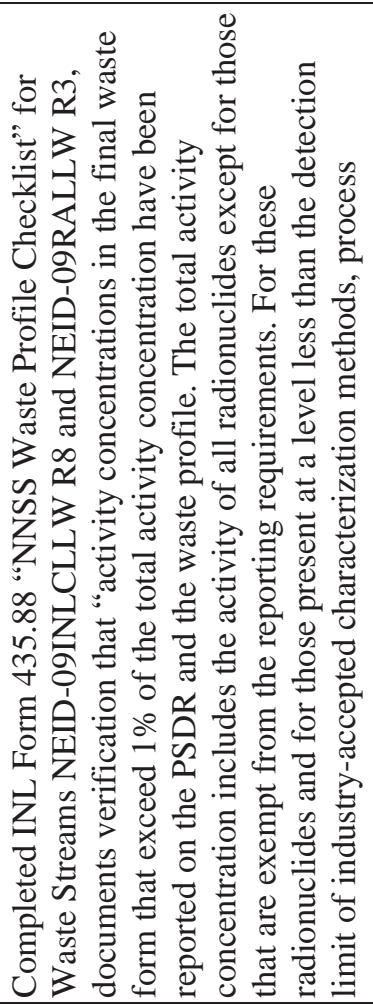 \\
\hline 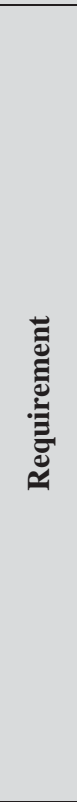 & 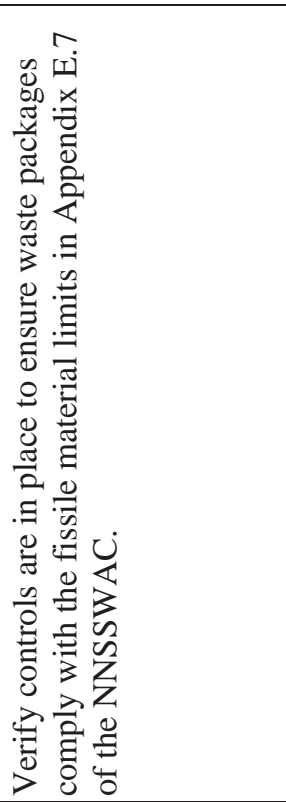 & 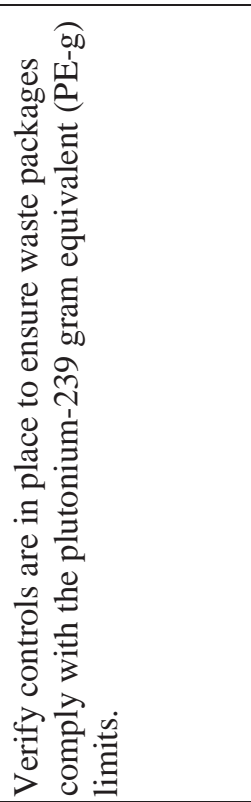 & & 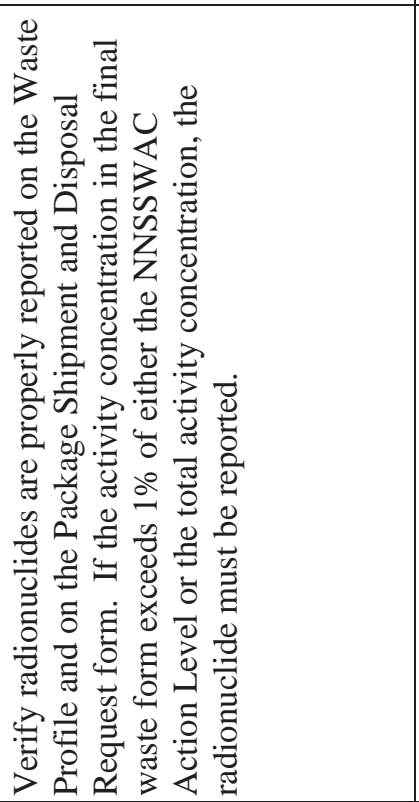 & 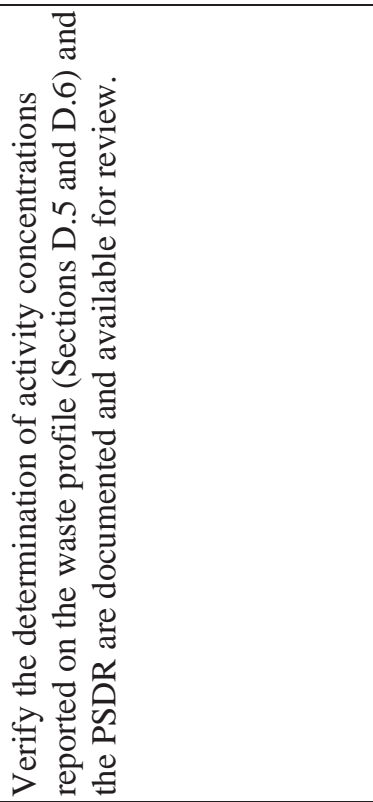 \\
\hline מֶّ & ָें & $\begin{array}{l}\tilde{N} \\
\stackrel{n}{n}\end{array}$ & & تָن & 더 \\
\hline$\dot{\Xi}$ & एु & $\dot{q}$ & & f & $\stackrel{\infty}{+}$ \\
\hline
\end{tabular}




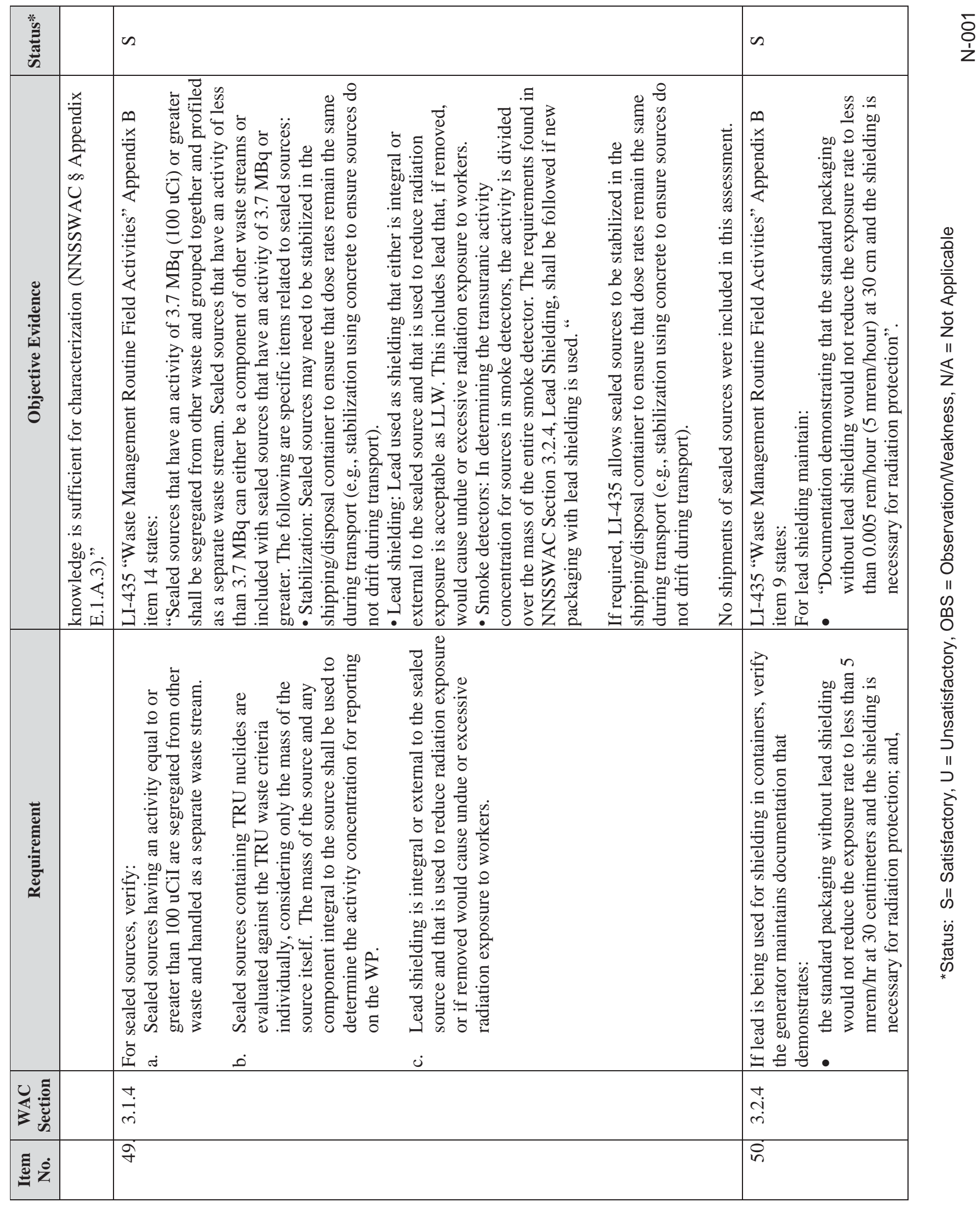




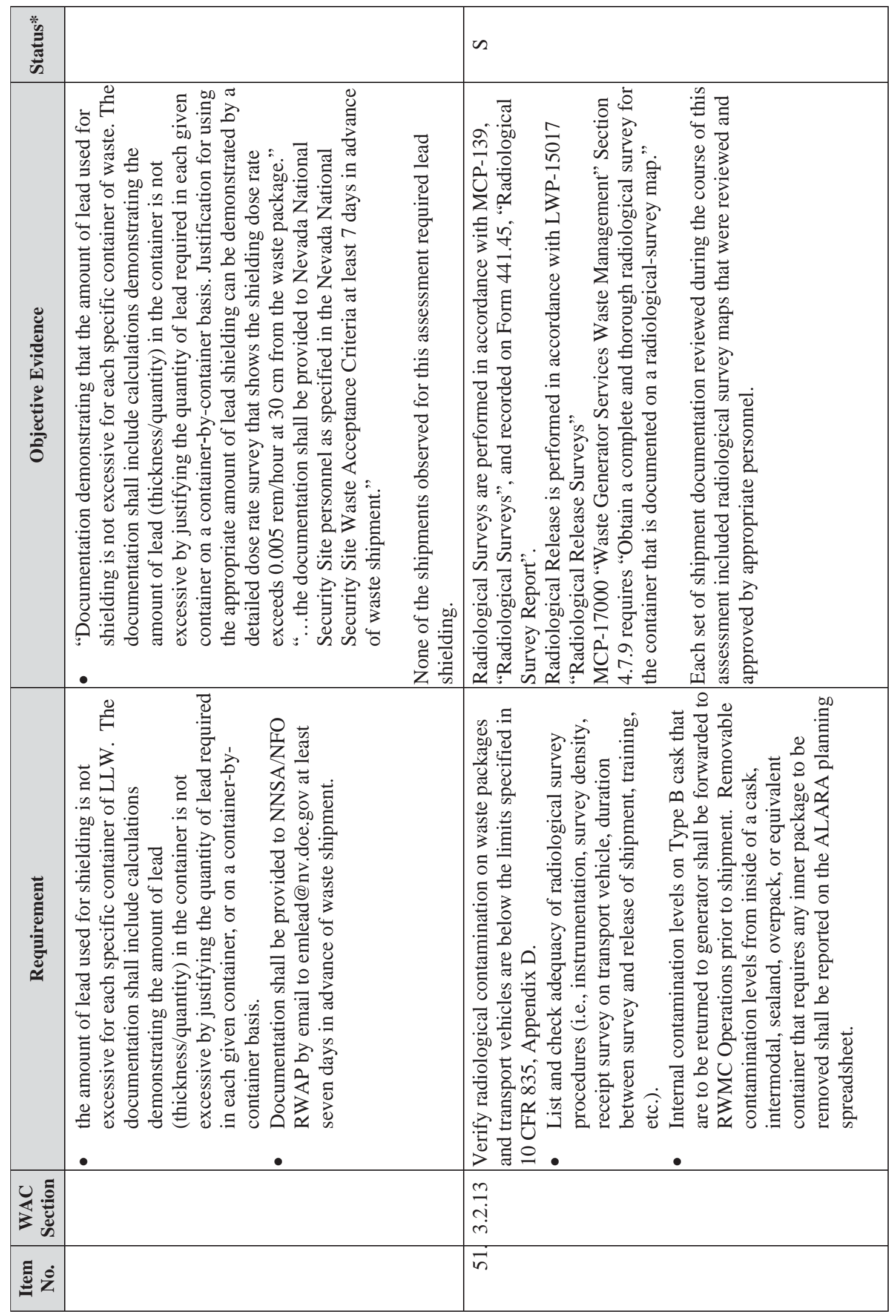

-1
8
2 


\section{Intentionally Blank}


Appendix F

NNSSWAC DOE/NV--325-16-00

Chemical Characterization Checklist 


\section{Intentionally Blank}



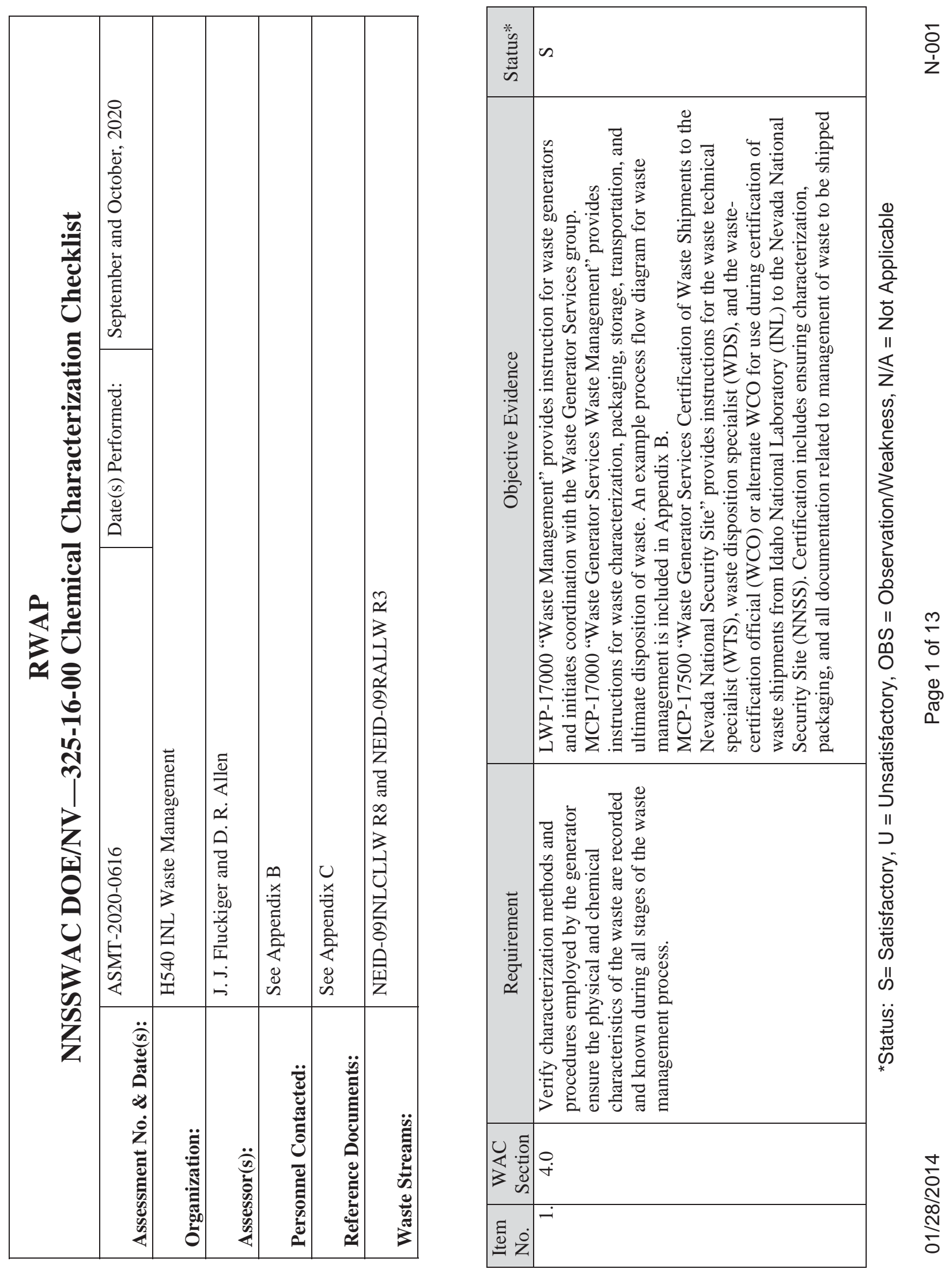


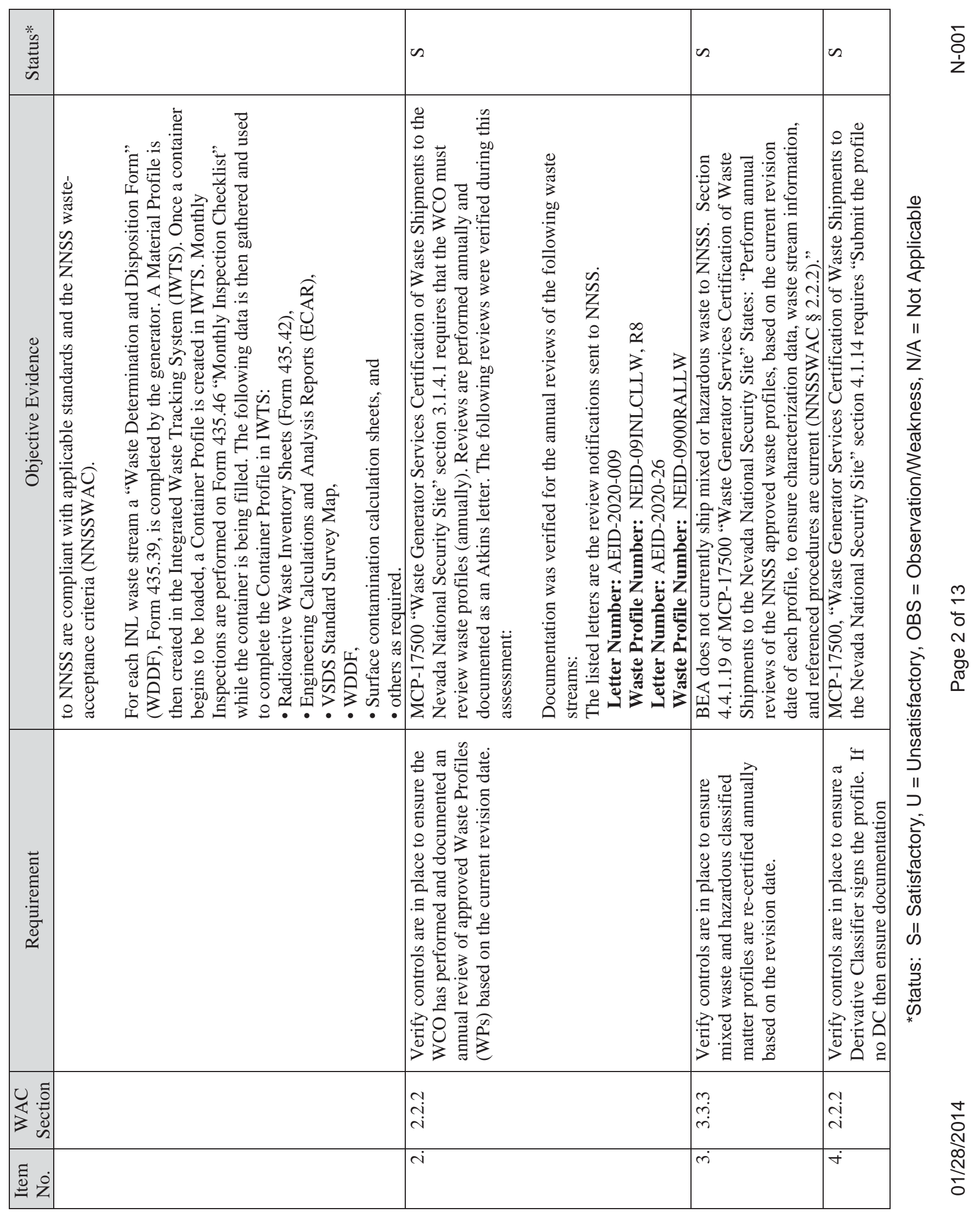




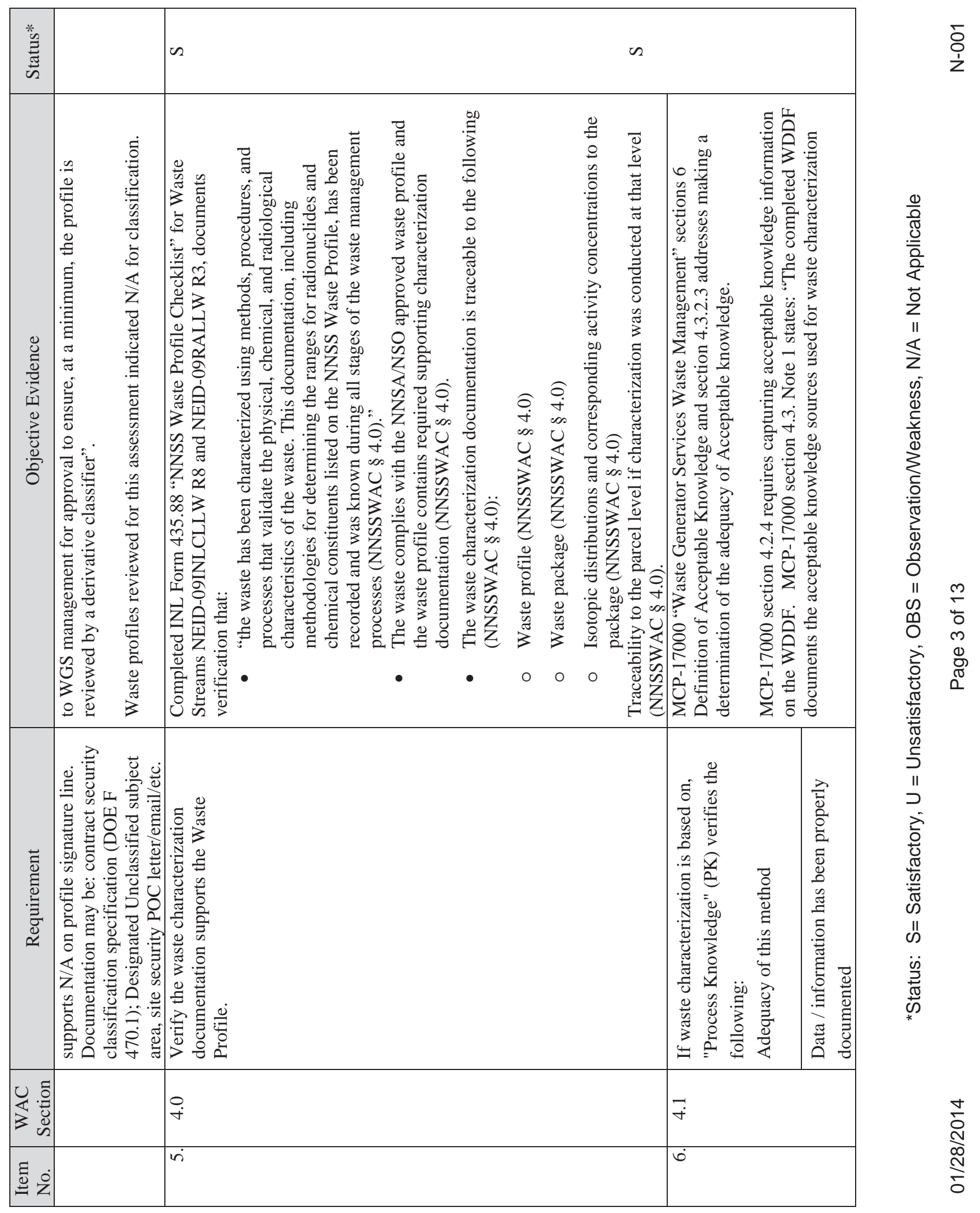




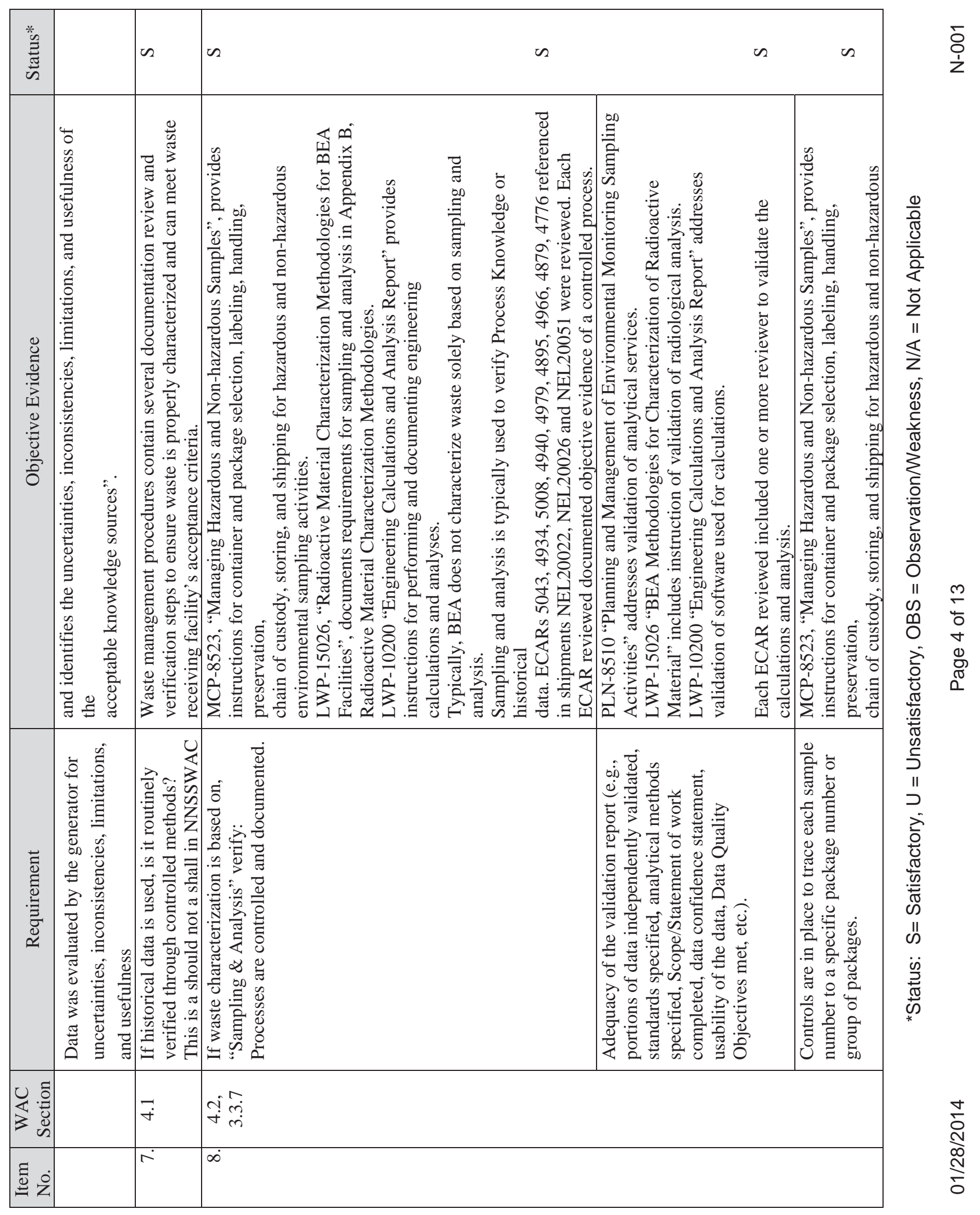




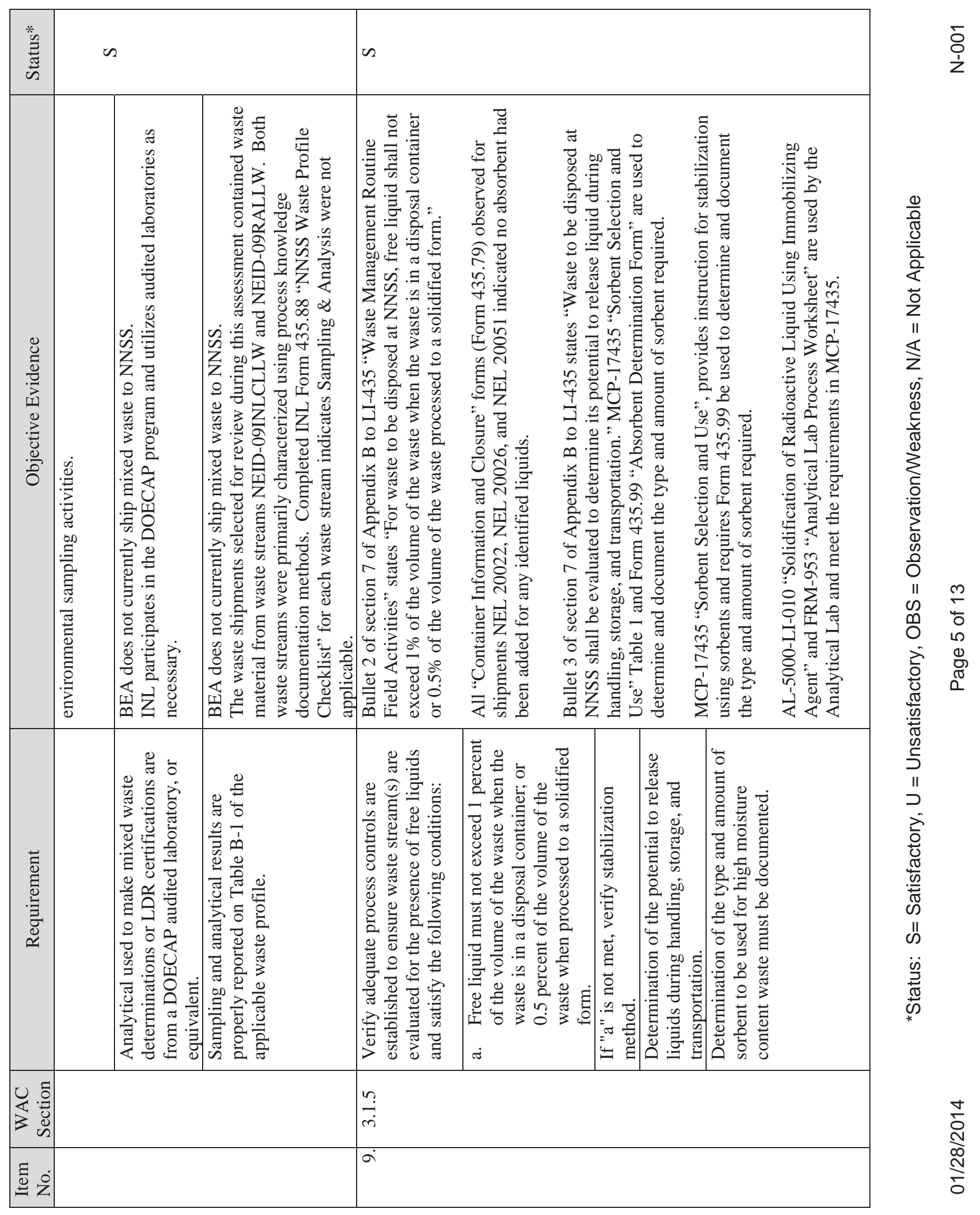




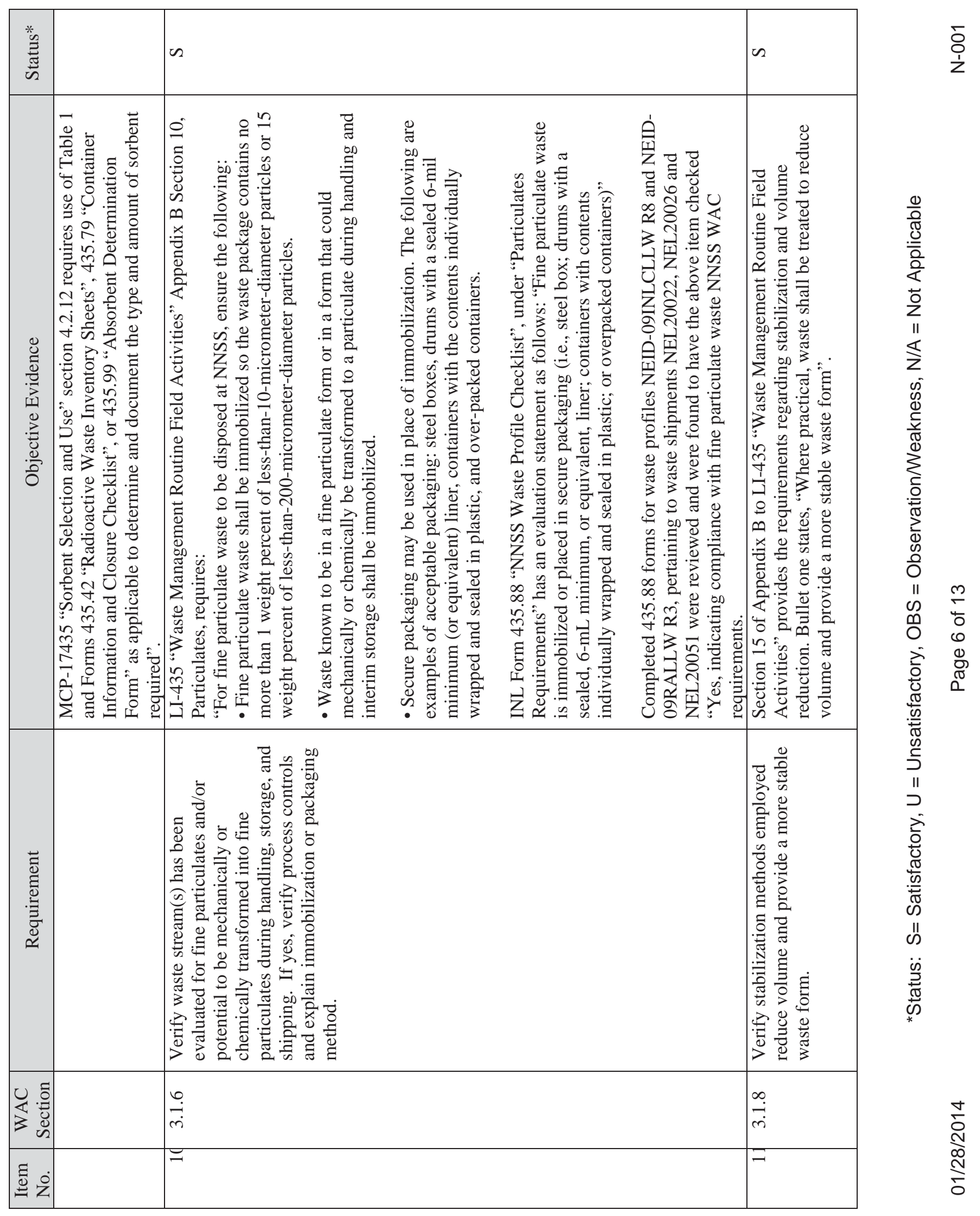




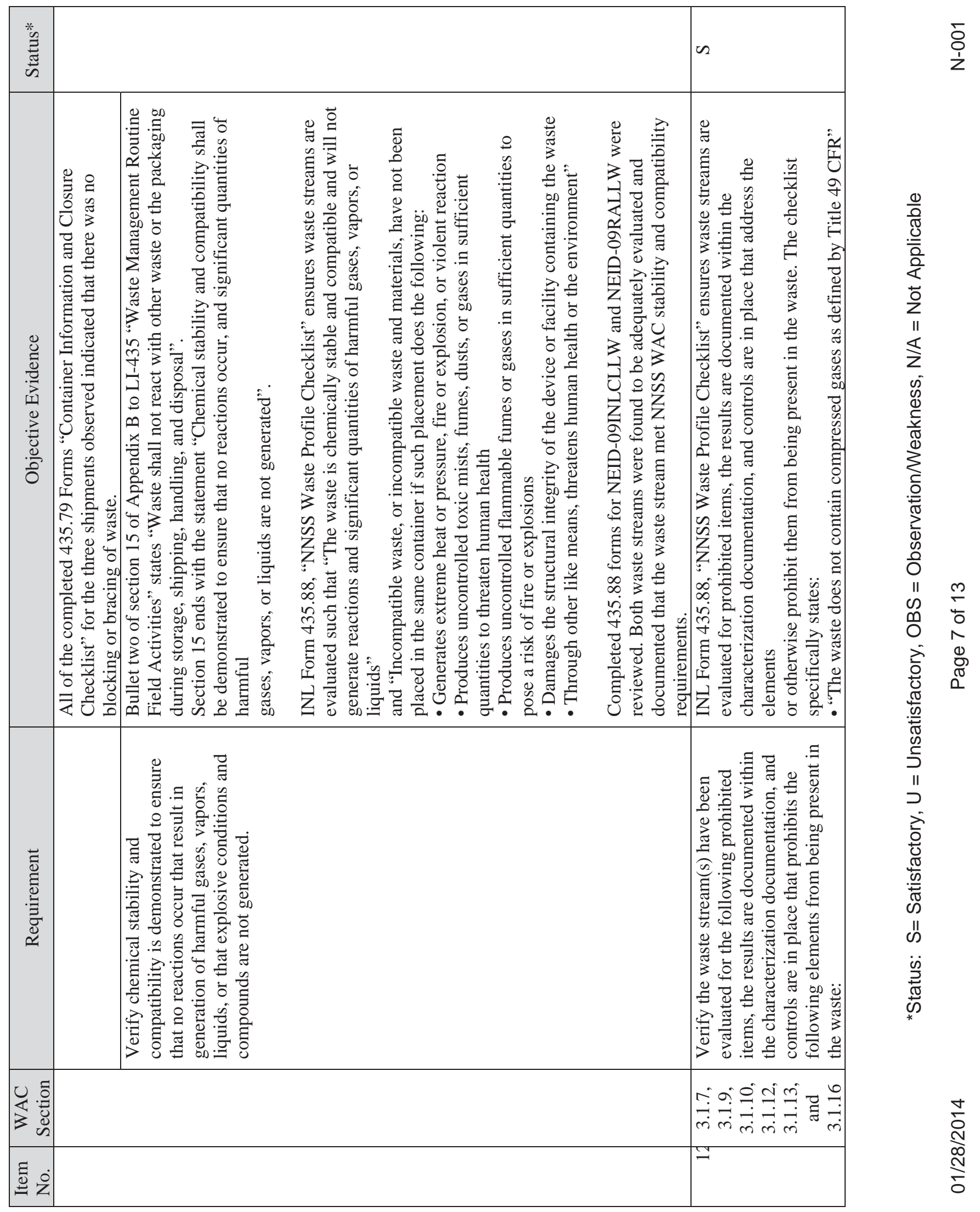




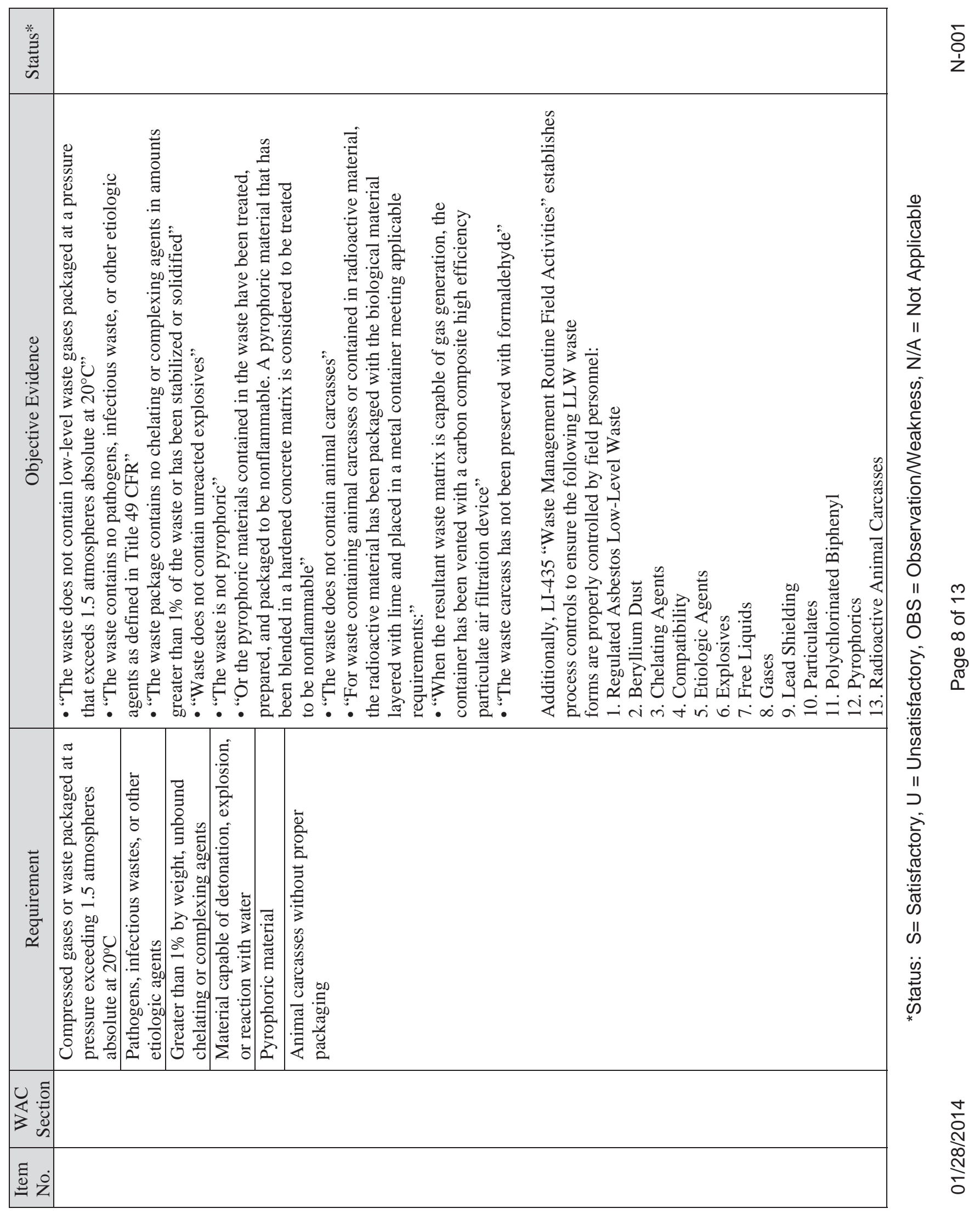




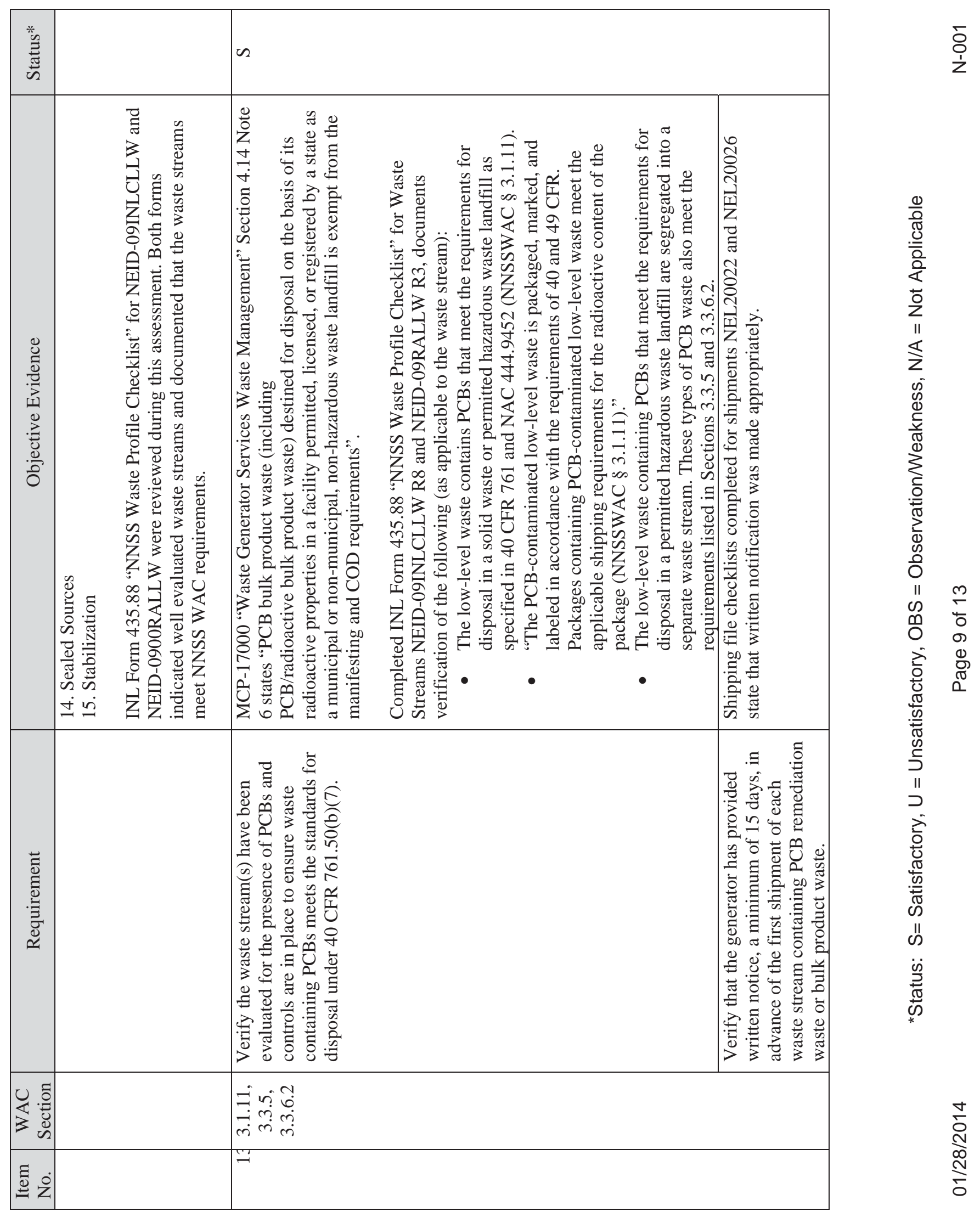




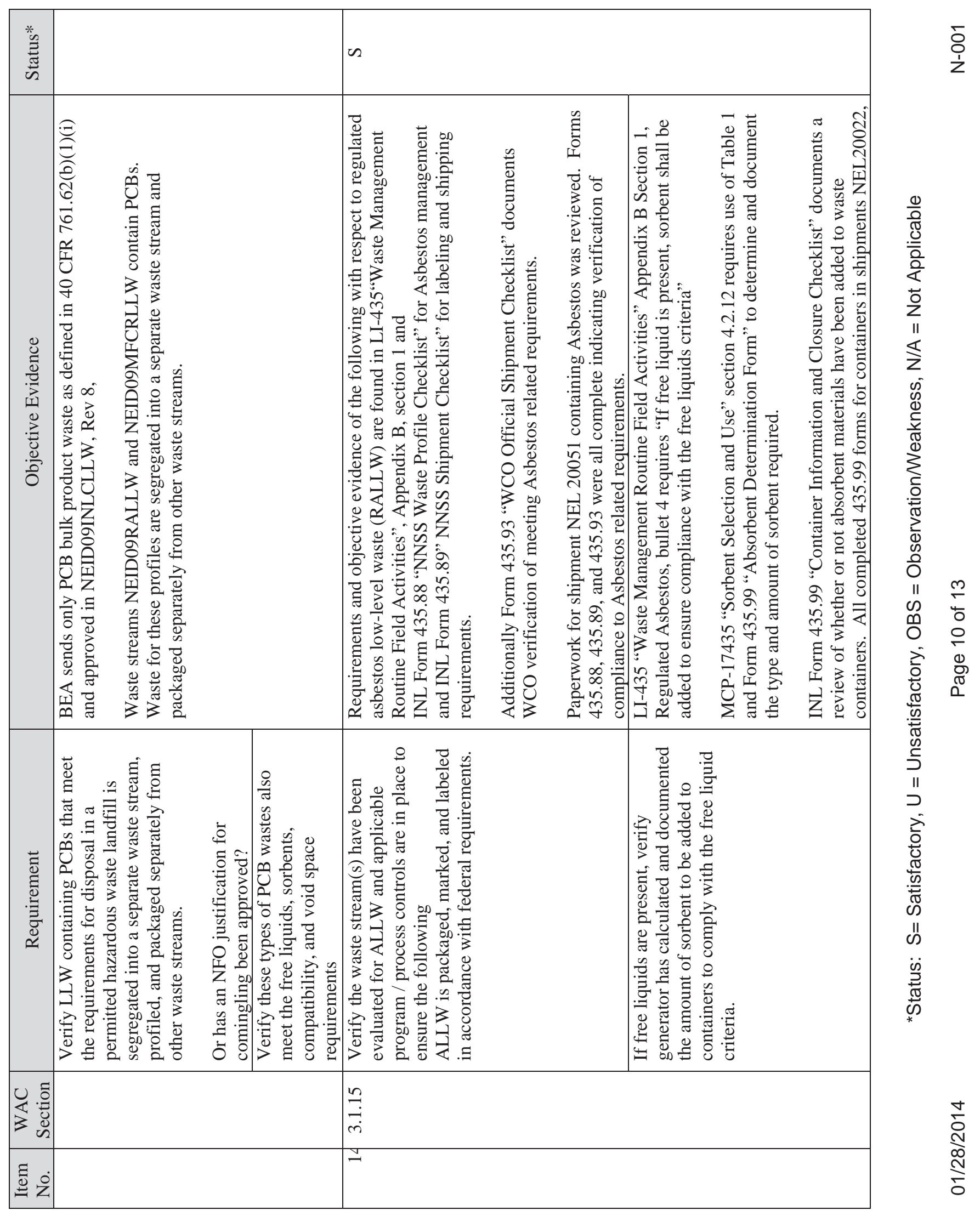




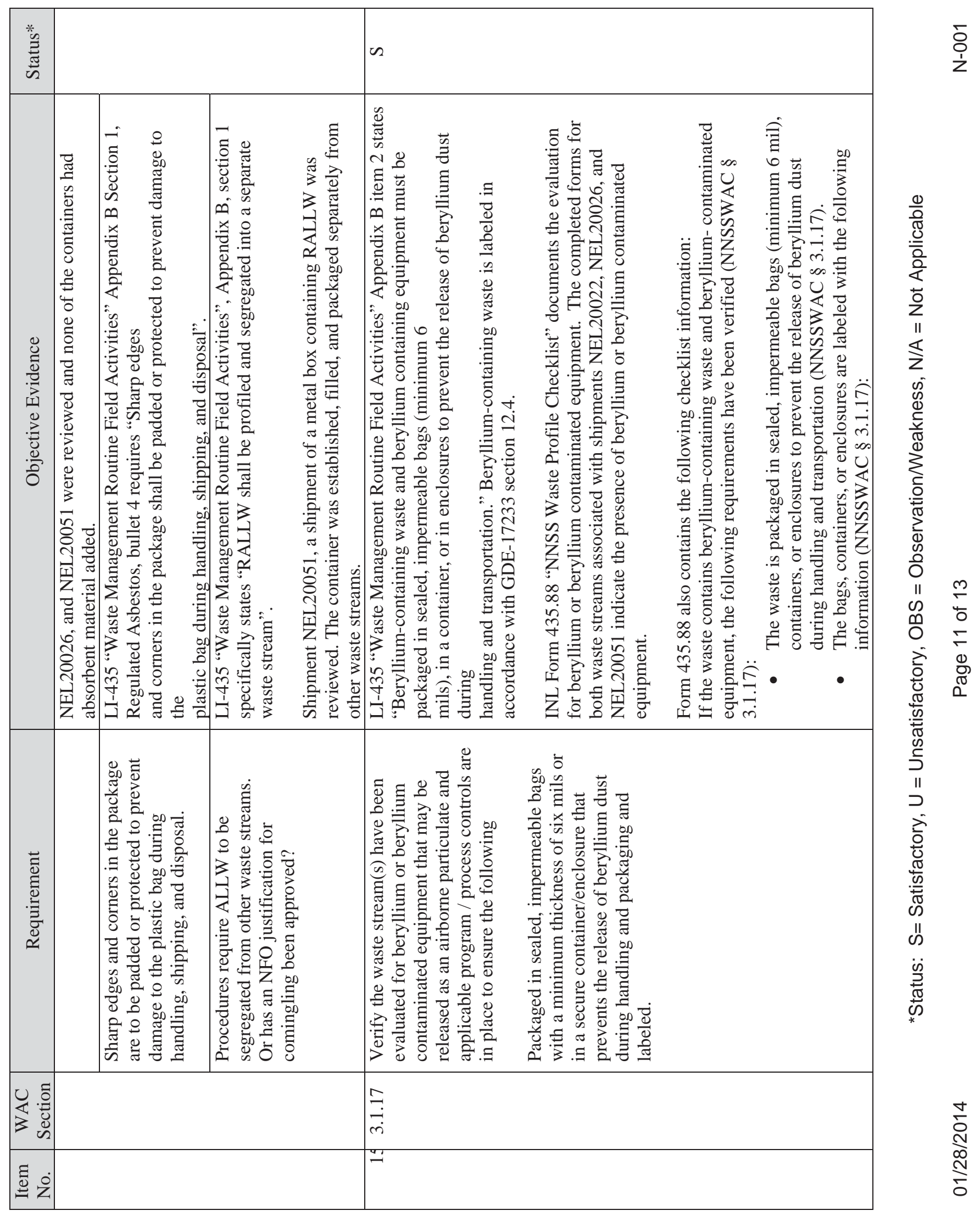




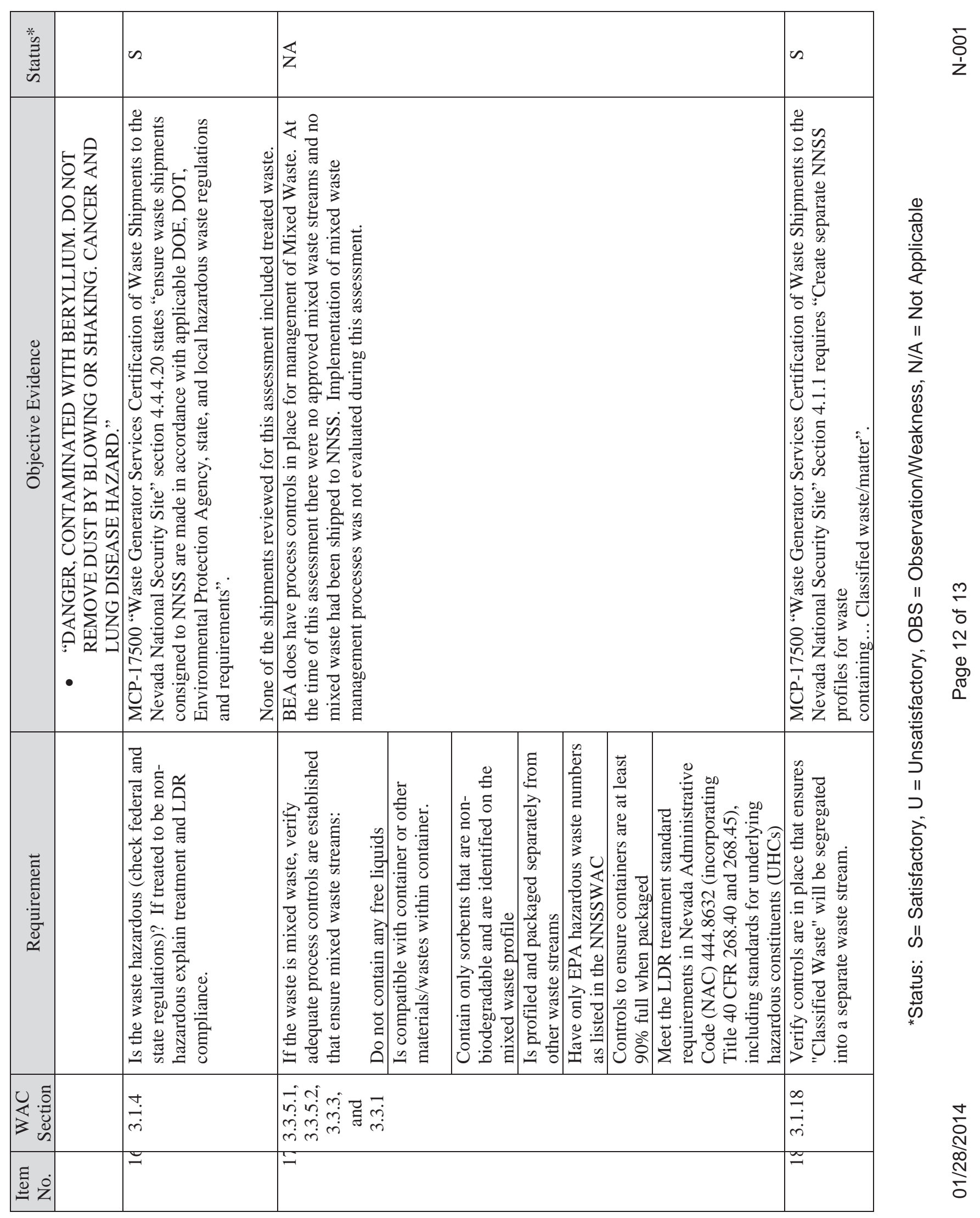




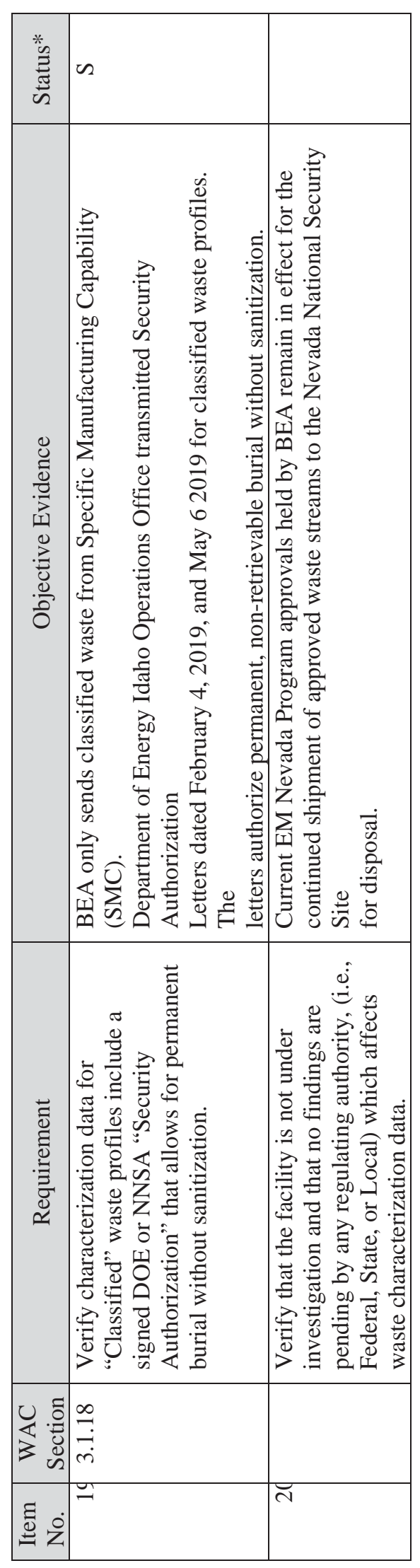

홀

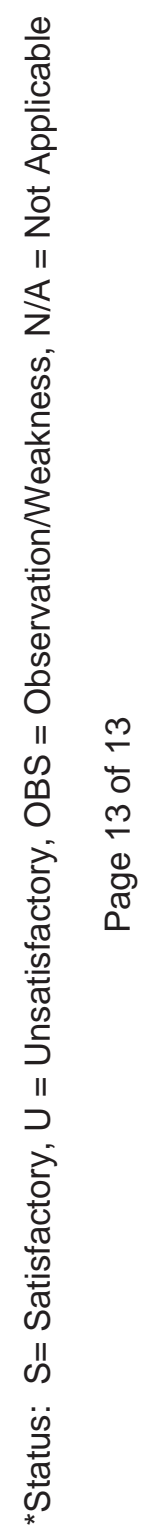

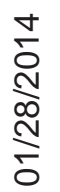




\section{Intentionally Blank}


Appendix G

NNSSWAC 325-16-00 Waste Traceability Checklist 


\section{Intentionally Blank}



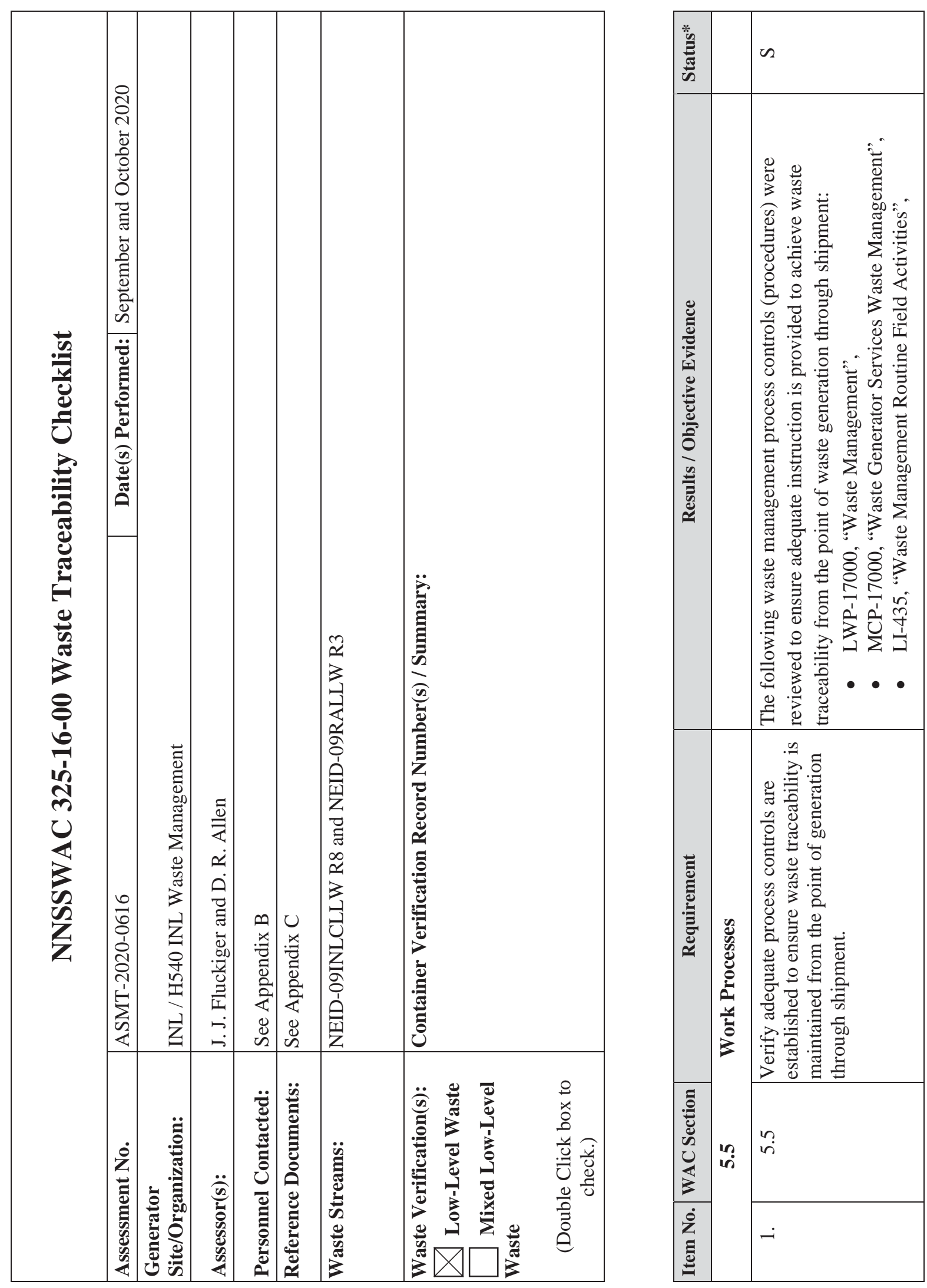


\begin{tabular}{|c|c|c|c|c|c|c|}
\hline 望 & & os & & & & in \\
\hline 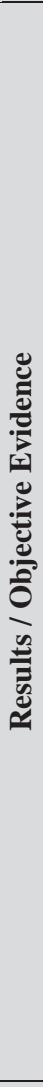 & 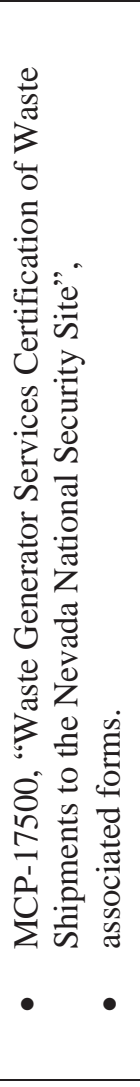 & 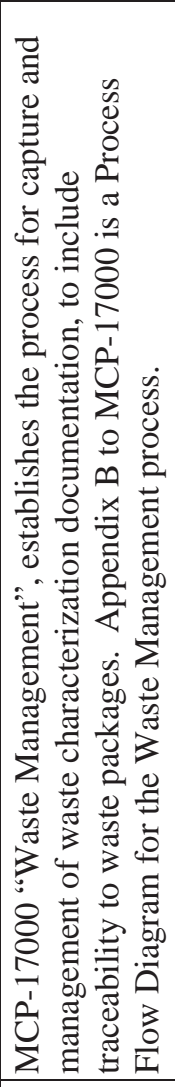 & 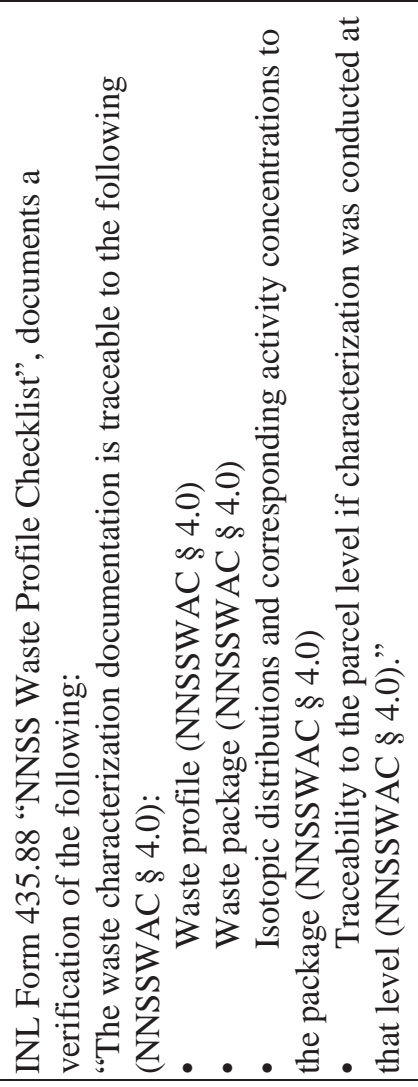 & 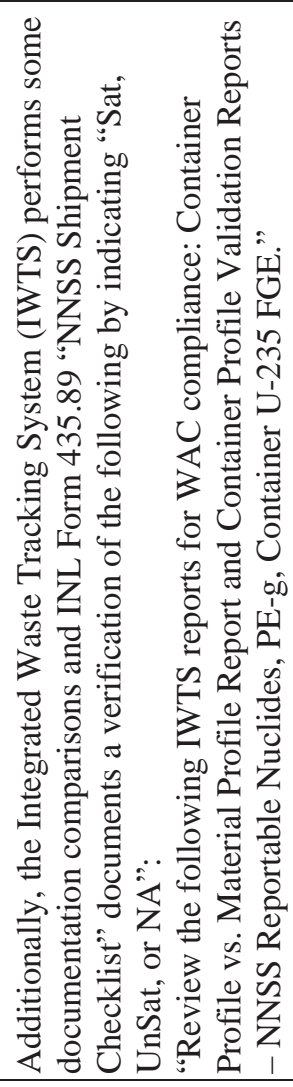 & 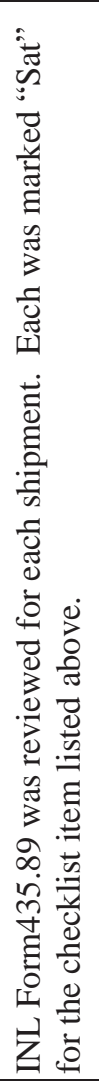 & 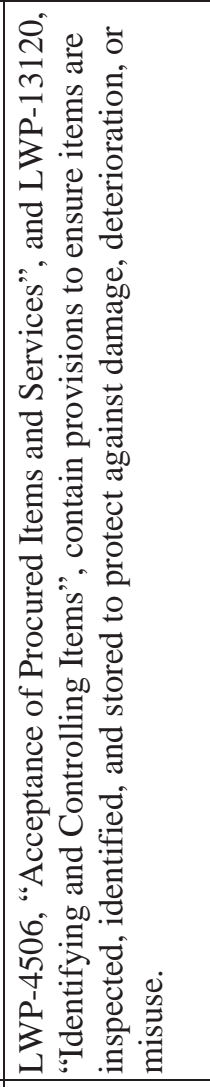 \\
\hline & & 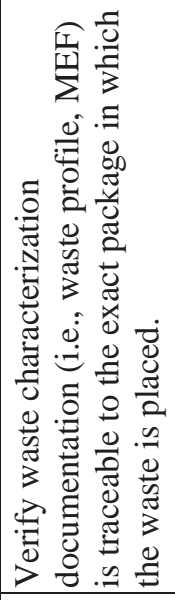 & & & & 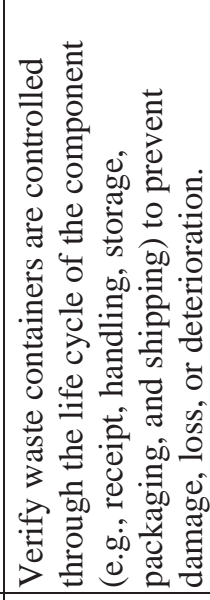 \\
\hline 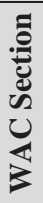 & & $\stackrel{\circ}{\forall}$ & & & & مُ \\
\hline 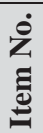 & & vi & & & & $\dot{m}$ \\
\hline
\end{tabular}




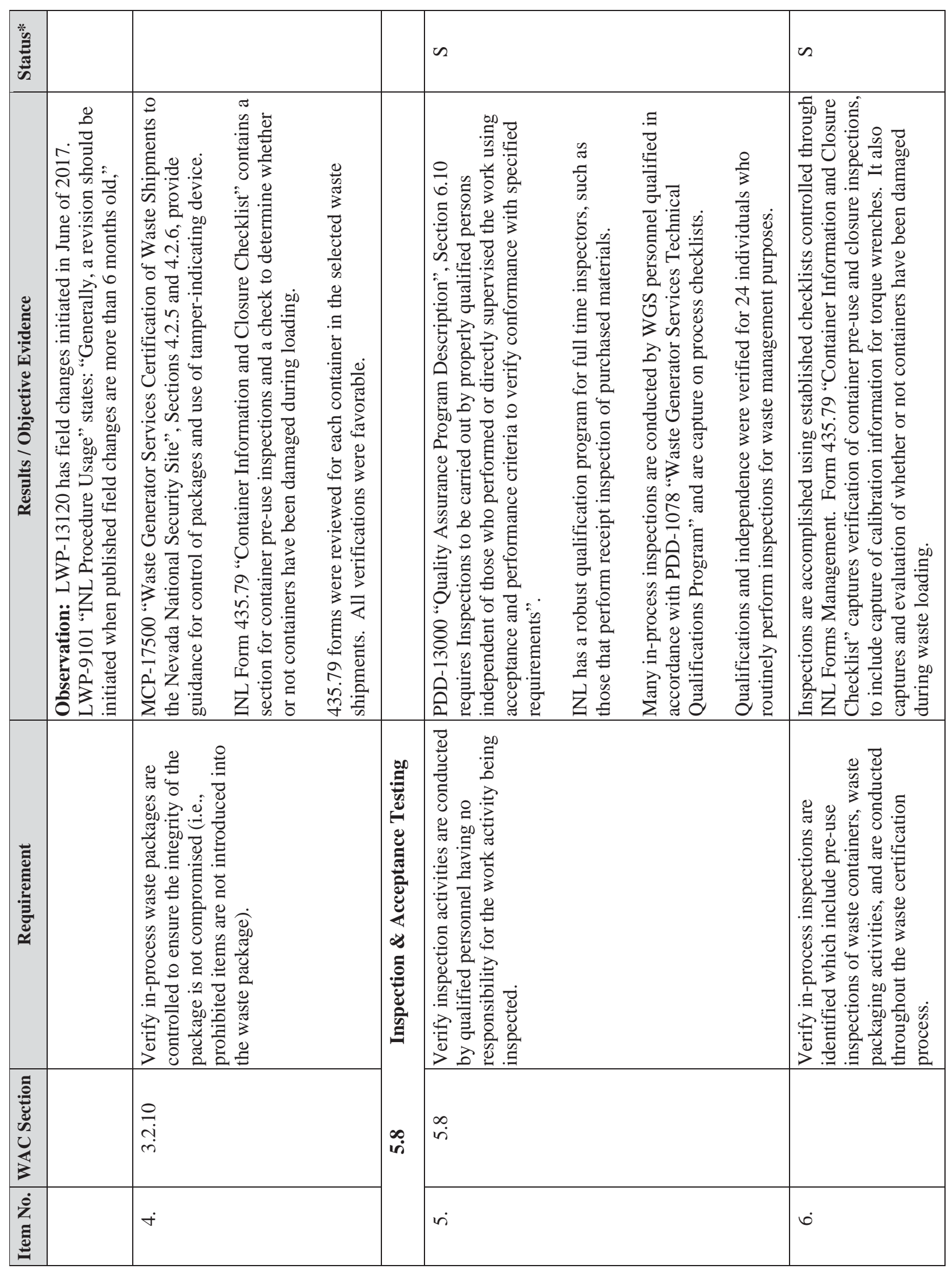




\begin{tabular}{|c|c|c|c|c|c|c|}
\hline 总 & & os & $n$ & & is & $n$ \\
\hline 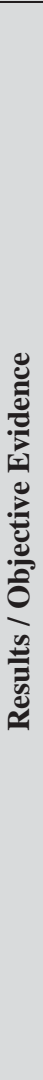 & 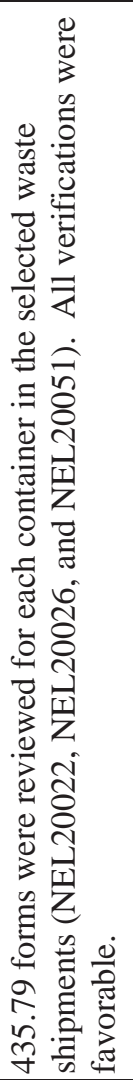 & 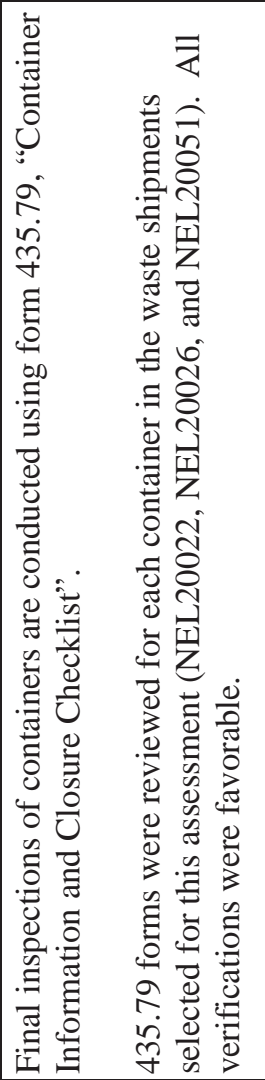 & 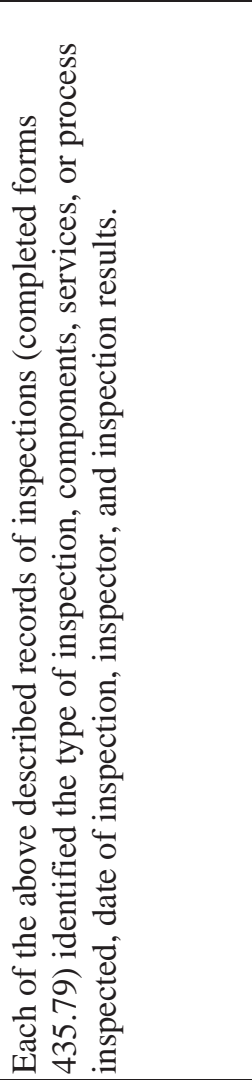 & & 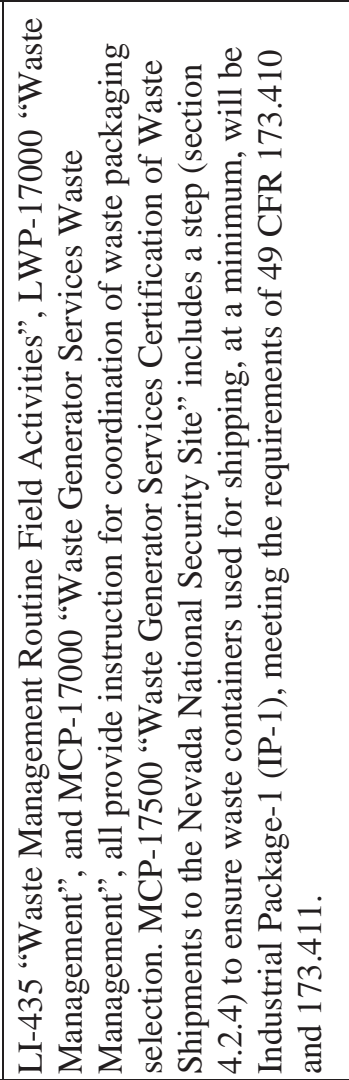 & 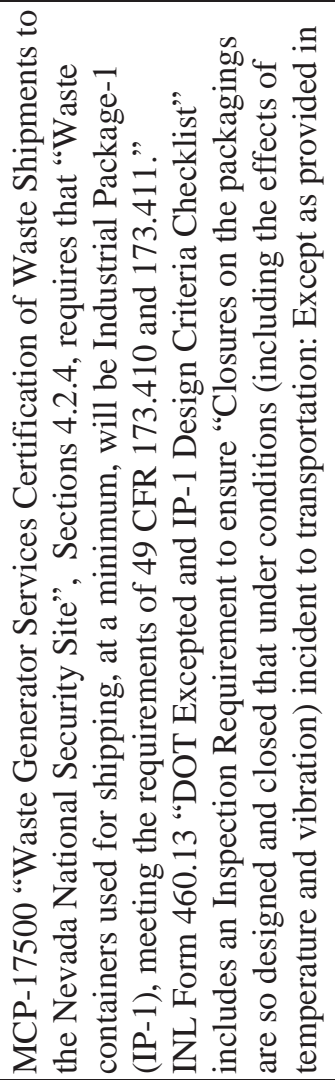 \\
\hline & & 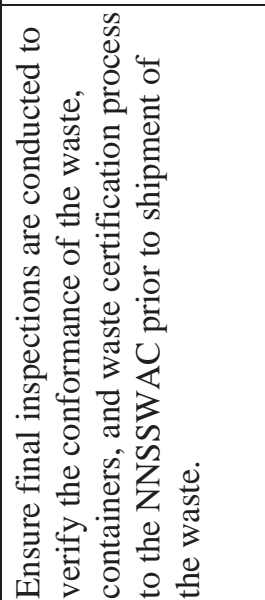 & 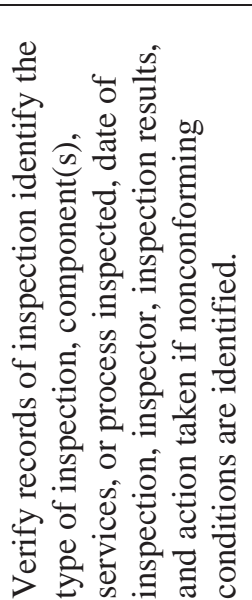 & 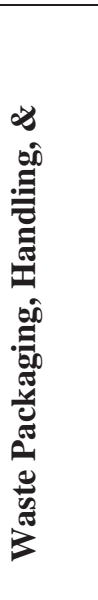 & 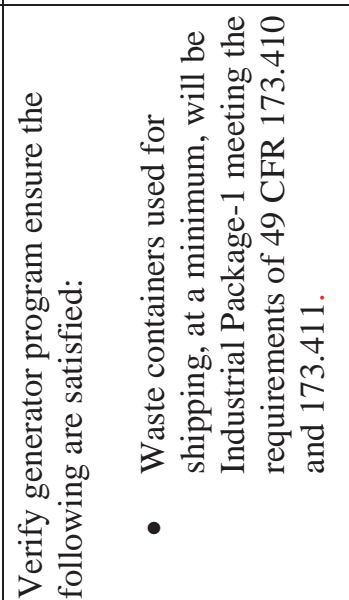 & 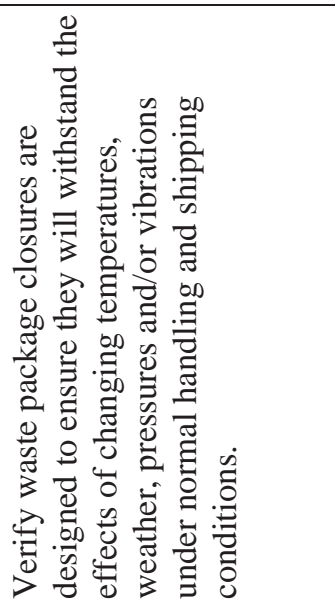 \\
\hline & & & & & 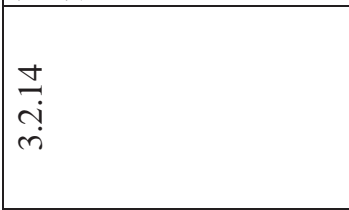 & 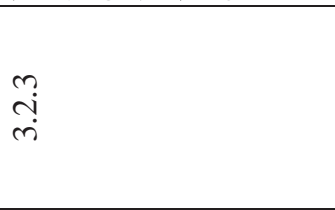 \\
\hline $\begin{array}{l}\mathrm{Z} \\
\bar{\Xi} \\
\underline{\Xi}\end{array}$ & & $\sim^{\circ}$ & $\infty$ & 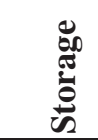 & $\sigma^{\circ}$ & $\stackrel{-}{\circ}$ \\
\hline
\end{tabular}




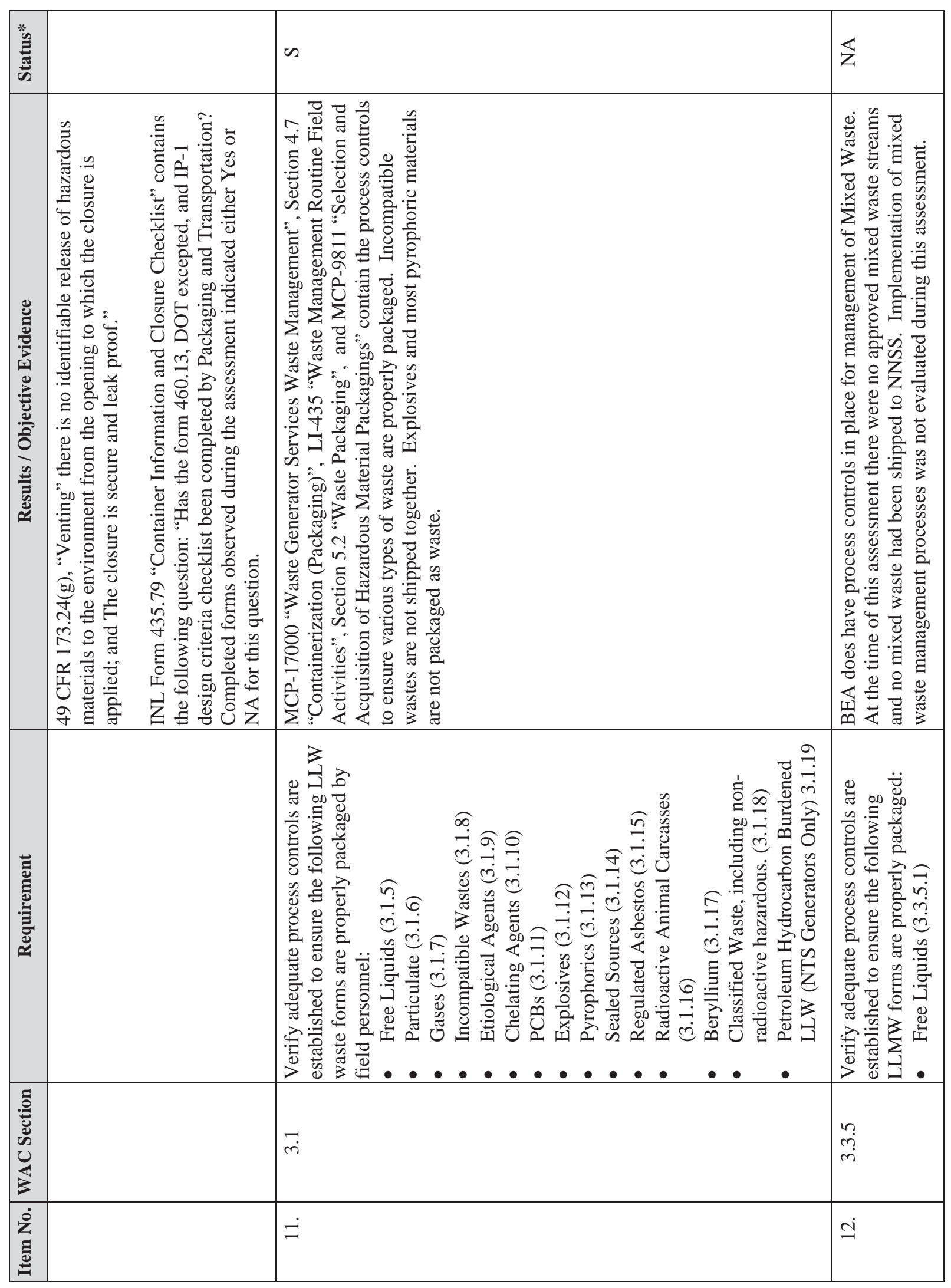




\begin{tabular}{|c|c|c|c|c|c|c|c|}
\hline 苞 & & ¿ & $\infty$ & & & & $n$ \\
\hline 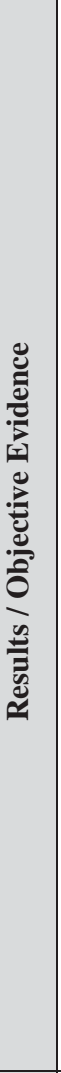 & & 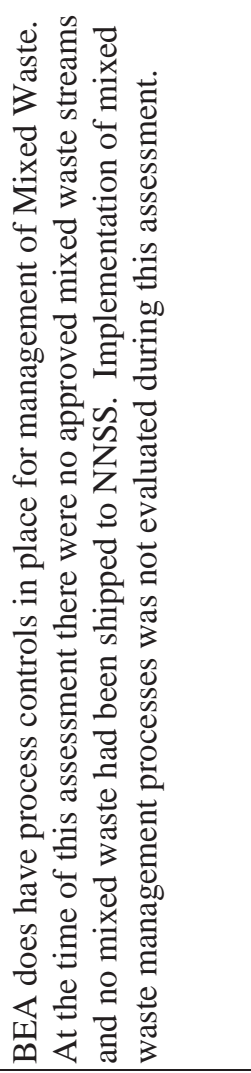 & 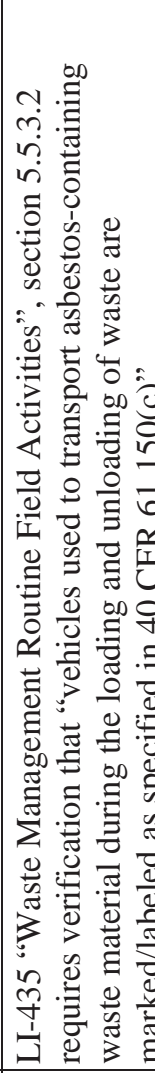 & 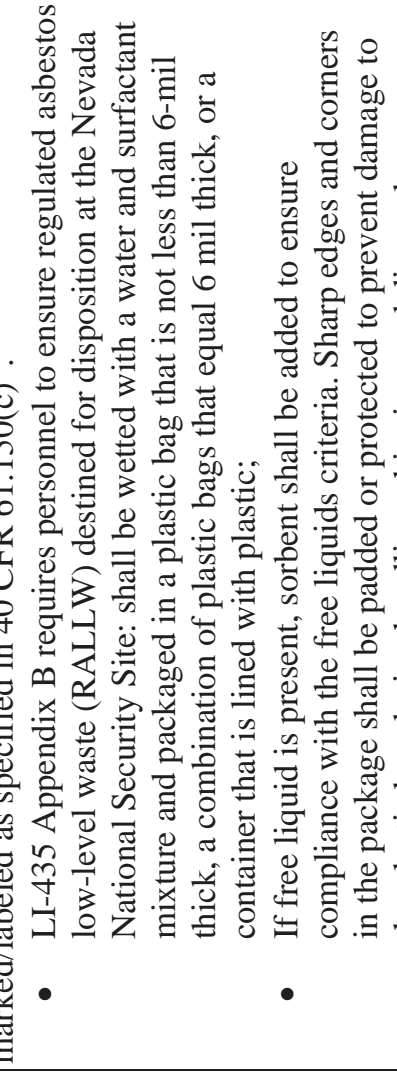 & 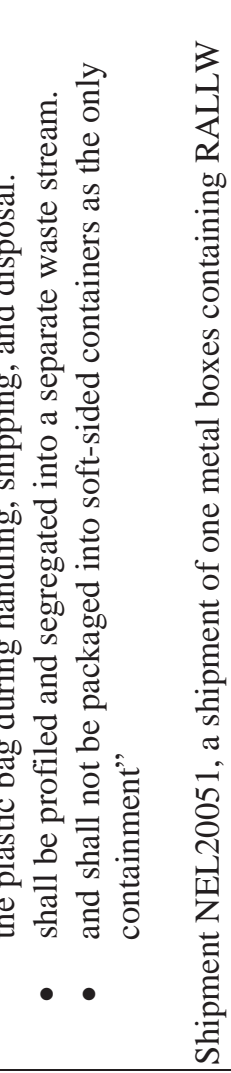 & 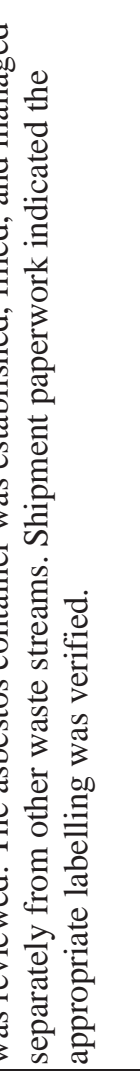 & 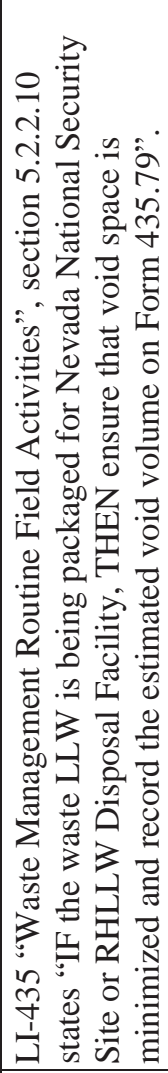 \\
\hline 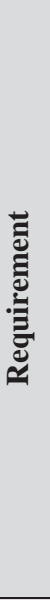 & 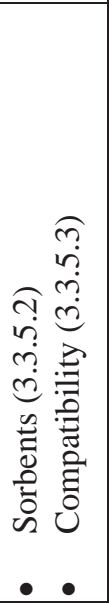 & 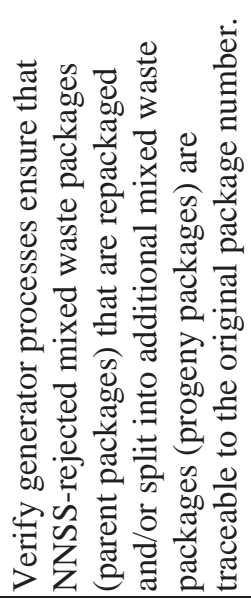 & 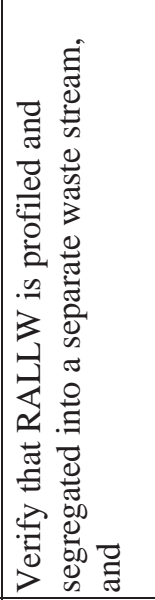 & 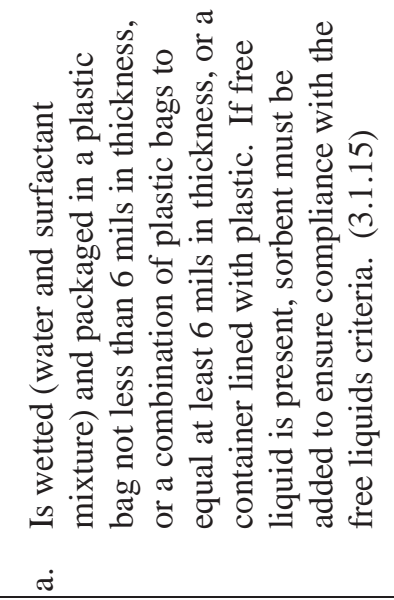 & 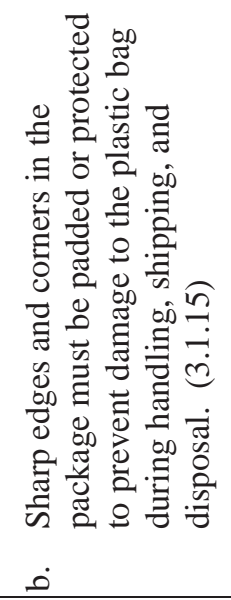 & 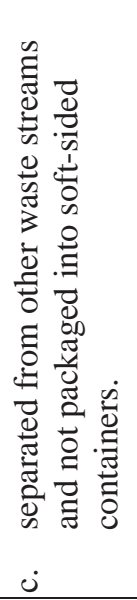 & 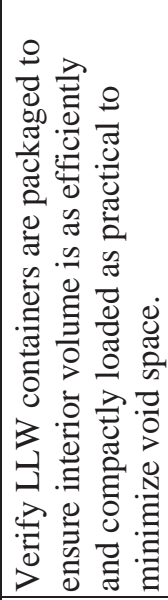 \\
\hline & & $\begin{array}{l}\text { N̦ } \\
\infty \\
\\
\tilde{m}\end{array}$ & 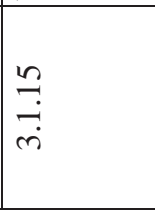 & & & & $\begin{array}{l}\vec{j} \\
\hat{n} \\
\dot{n}\end{array}$ \\
\hline 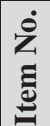 & & $\stackrel{m}{\rightarrow}$ & 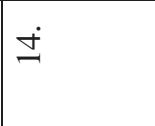 & & & & $\stackrel{L 2}{\sim}$ \\
\hline
\end{tabular}




\begin{tabular}{|c|c|c|c|c|c|c|c|c|}
\hline 苐 & & $n$ & $n$ & & & & & ns \\
\hline & 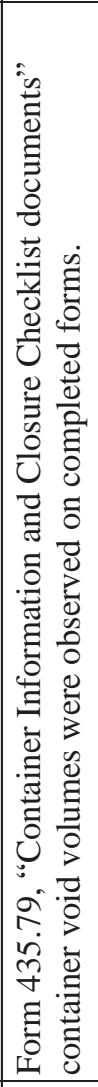 & 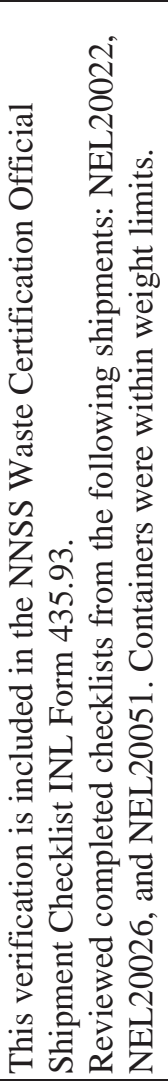 & 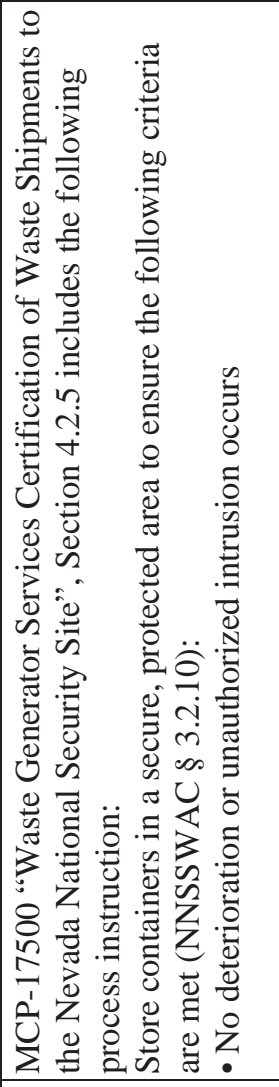 & 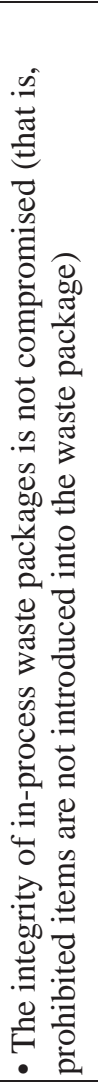 & 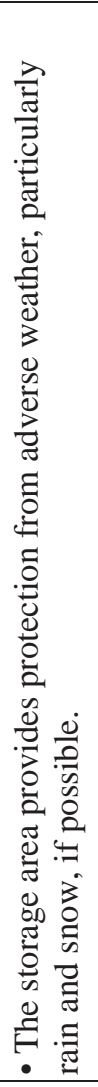 & 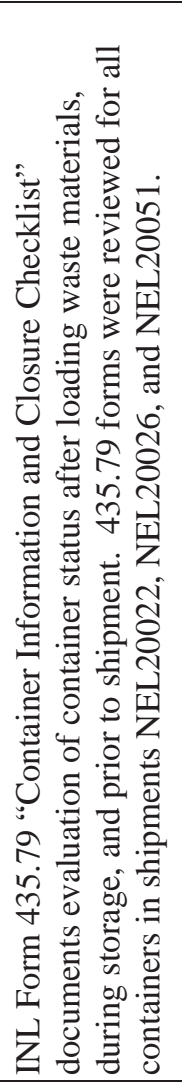 & & 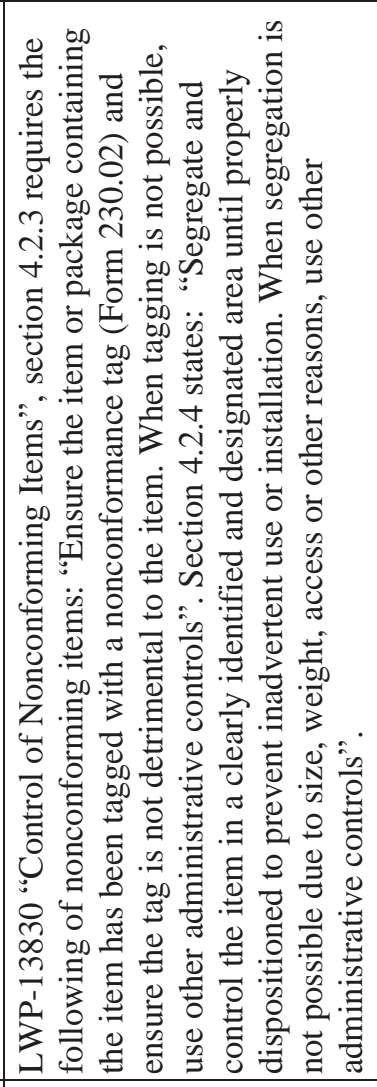 \\
\hline & & 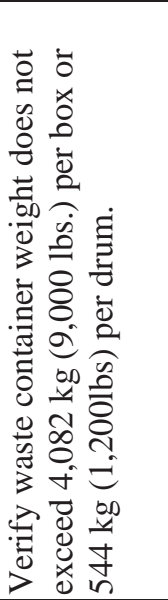 & \multicolumn{4}{|c|}{ 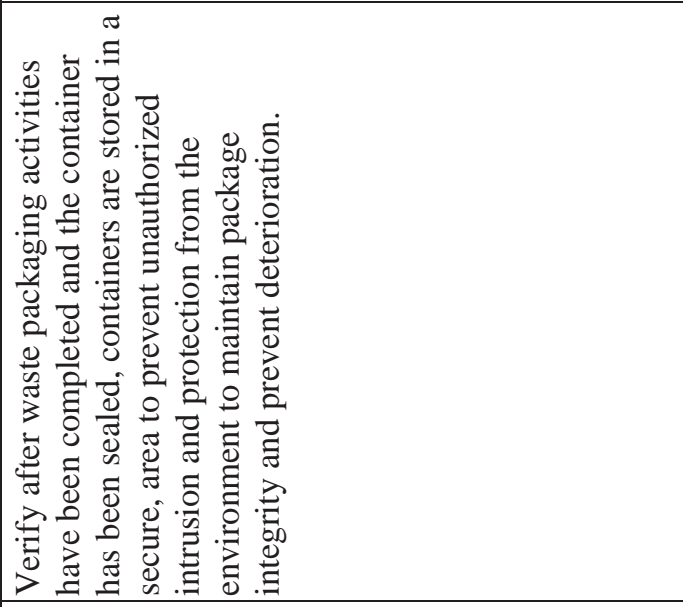 } & \multirow{3}{*}{ 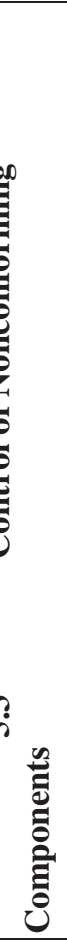 } & 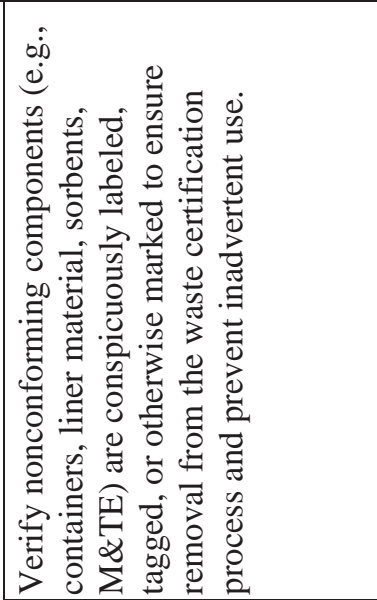 \\
\hline 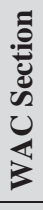 & & $\begin{array}{l}\infty \\
\text { Ny } \\
\text { n் }\end{array}$ & \multicolumn{4}{|l|}{$\begin{array}{l}\circ \\
\stackrel{0}{\sim} \\
\stackrel{n}{n} \\
\dot{m}\end{array}$} & & \begin{tabular}{|l}
$m$ \\
in
\end{tabular} \\
\hline $\begin{array}{l}Z \\
\text { 离 }\end{array}$ & & $\stackrel{\bullet}{\sim}$ & \multicolumn{4}{|l|}{$\stackrel{-}{-}$} & & $\stackrel{\infty}{\sim}$ \\
\hline
\end{tabular}




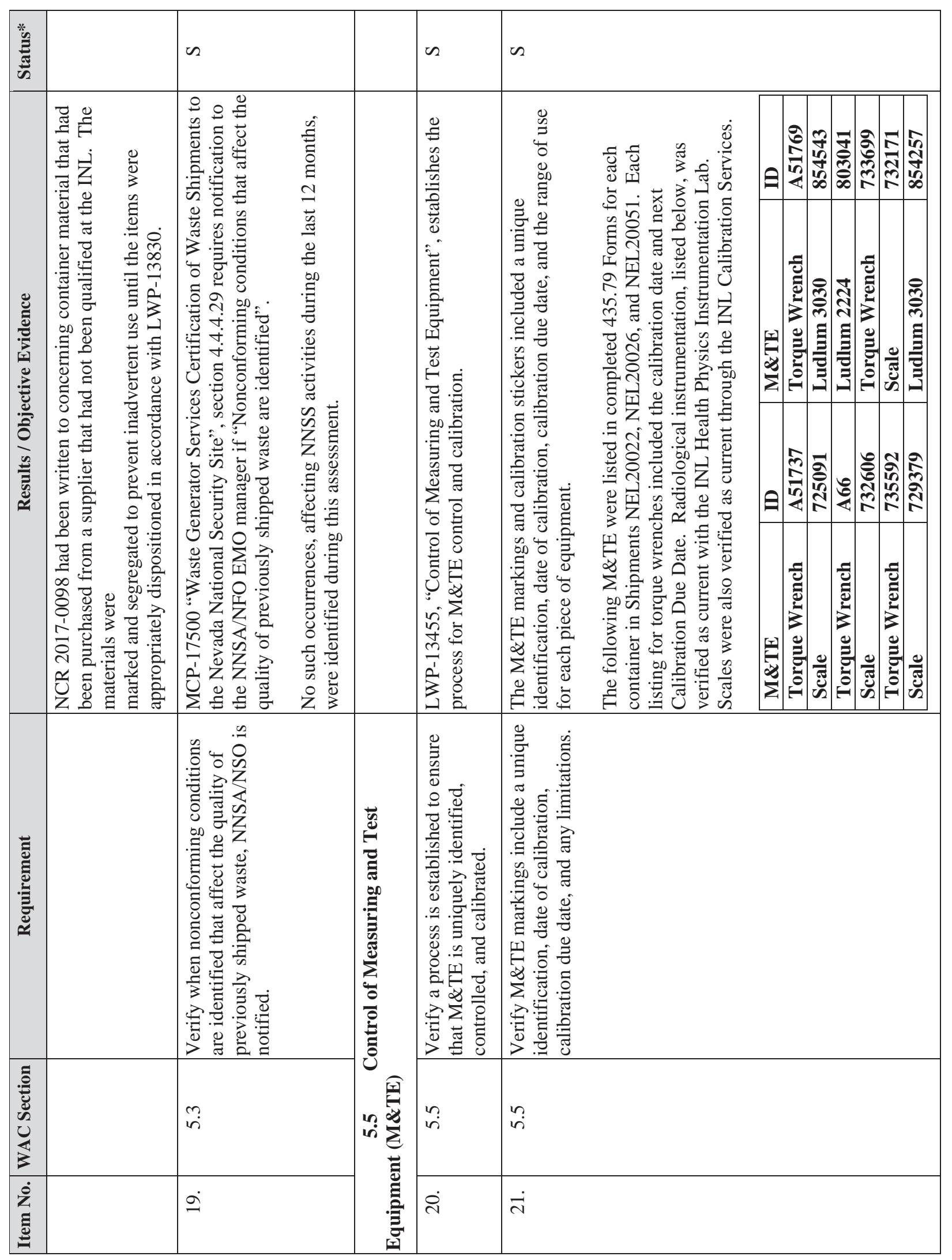




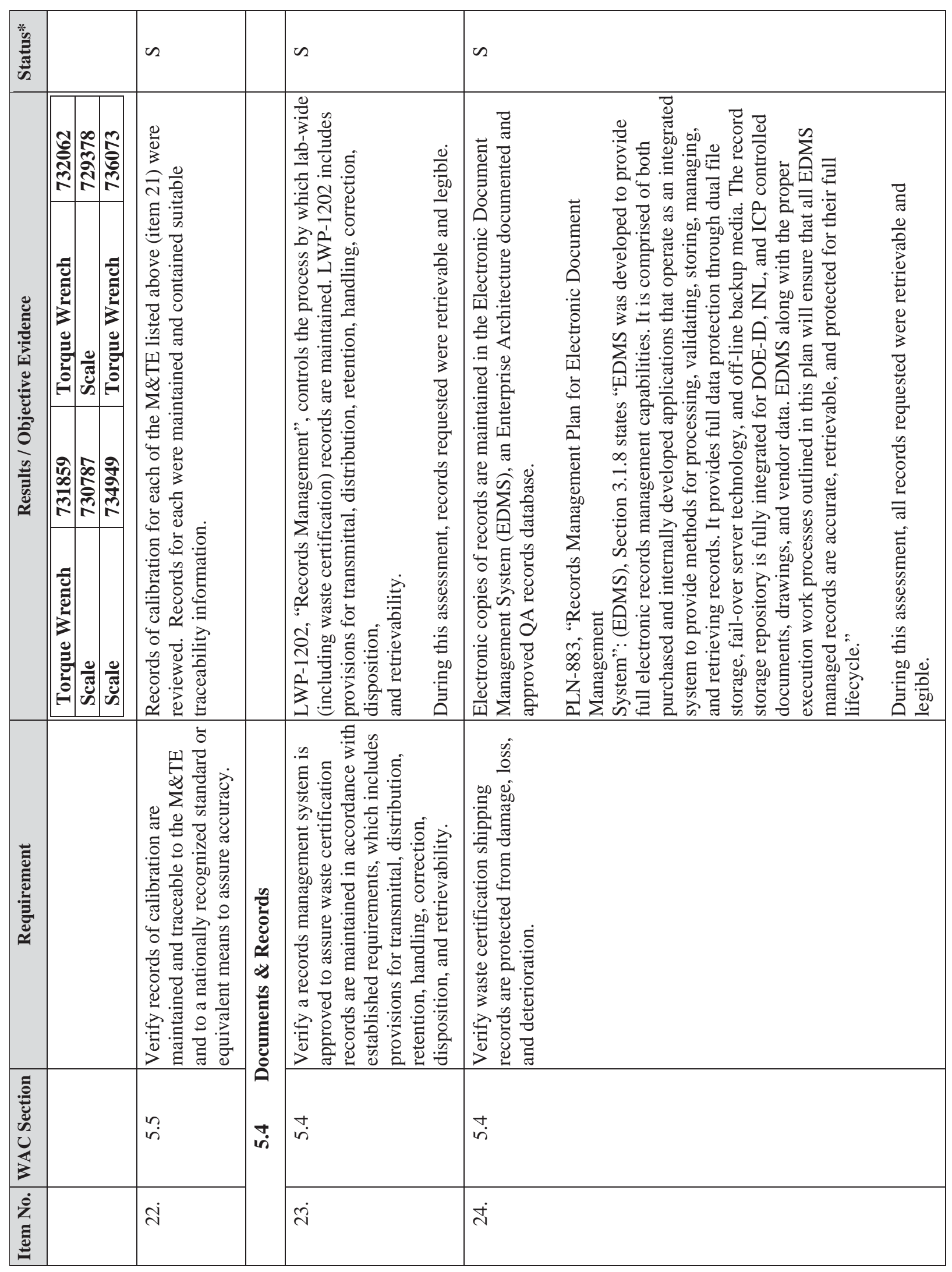




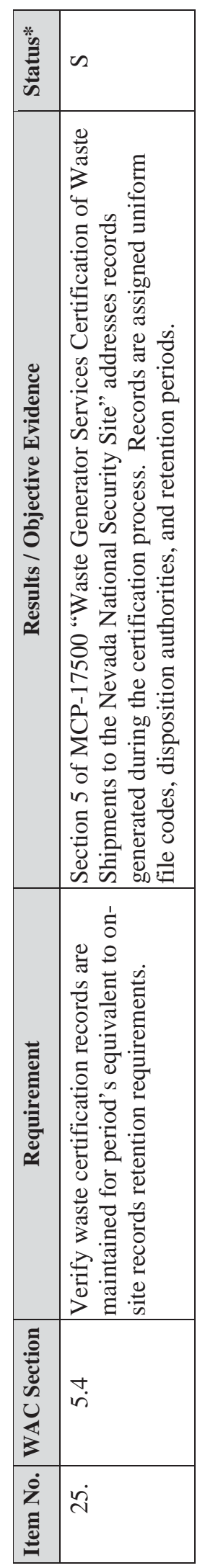


Appendix $\mathrm{H}$

NNSSWAC 325-16-00 Waste Transportation Assessment Checklist 


\section{Intentionally Blank}




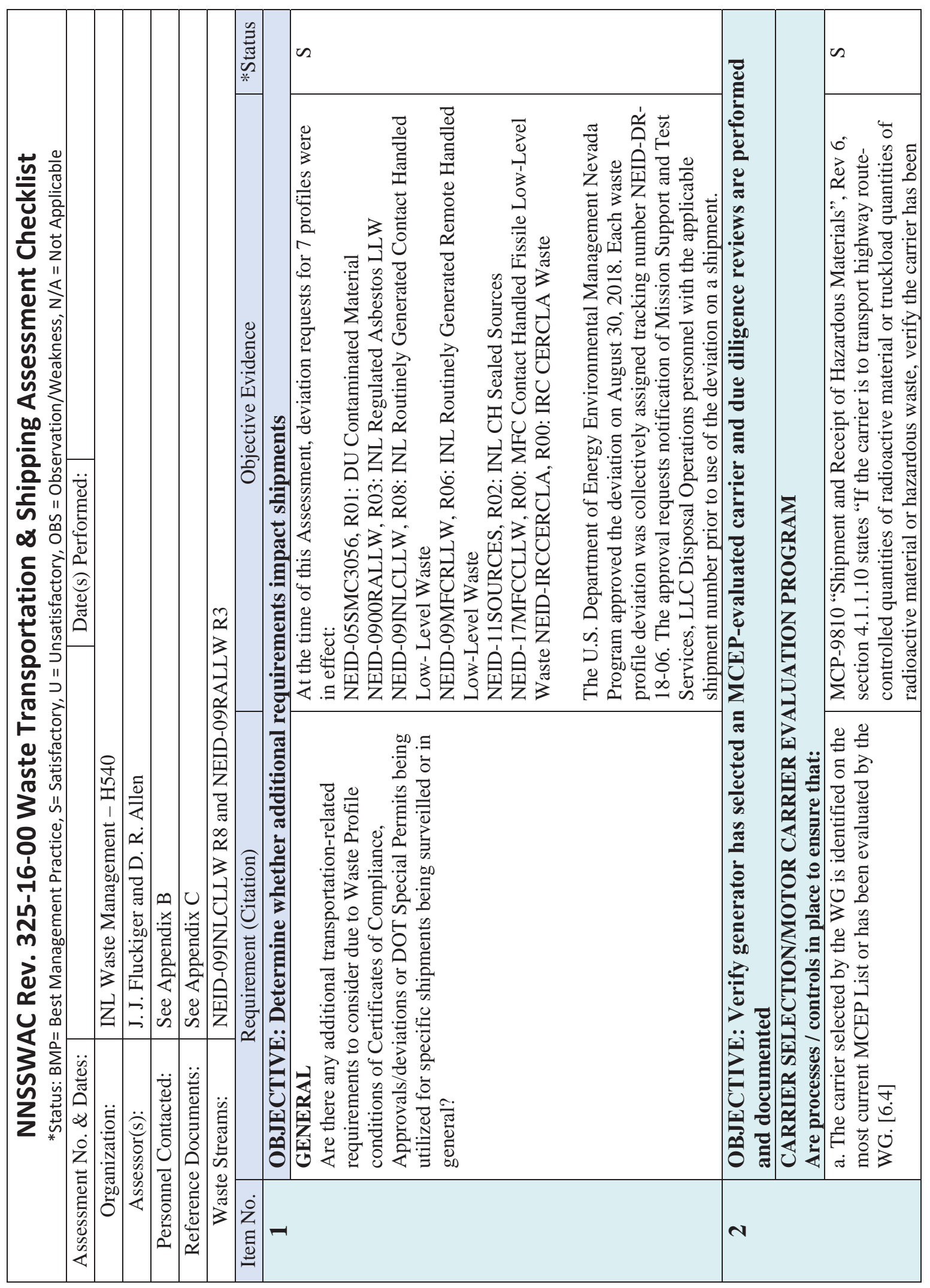




\begin{tabular}{|c|c|c|c|c|c|c|c|c|}
\hline & & is & & $\mathbb{z}$ & $\mid \overleftarrow{z}$ & is & & \\
\hline \multirow[t]{2}{*}{ 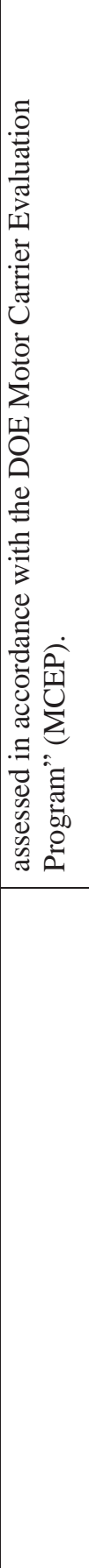 } & 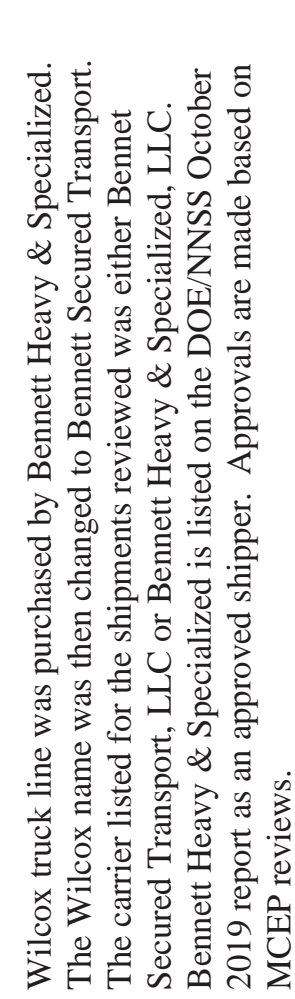 & 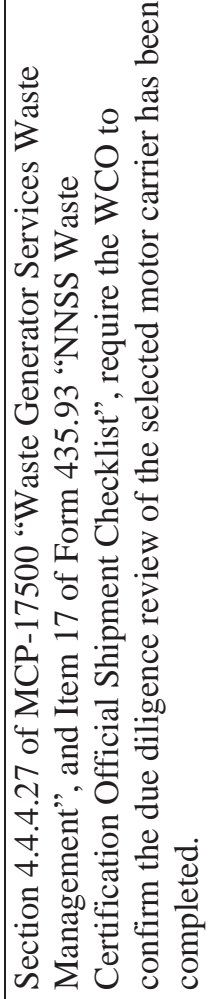 & 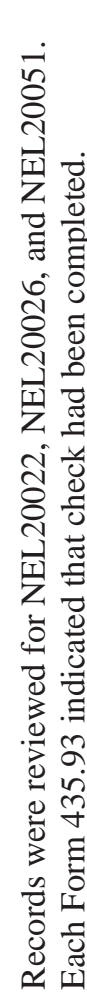 & 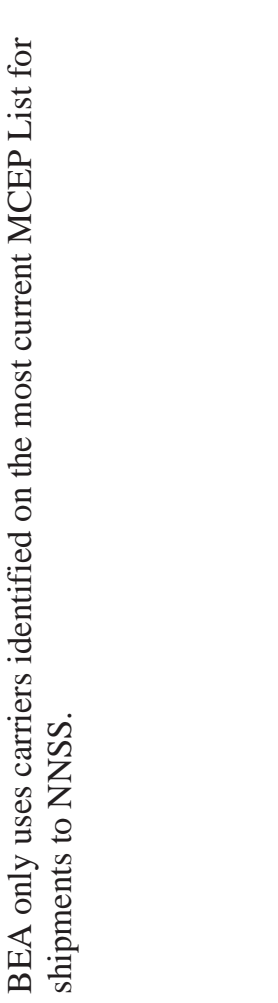 & 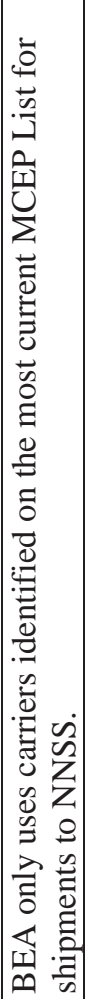 & 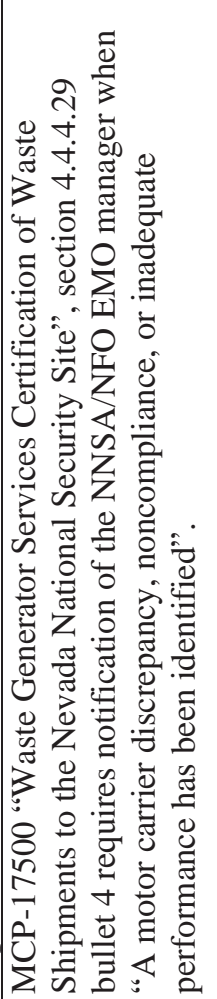 & 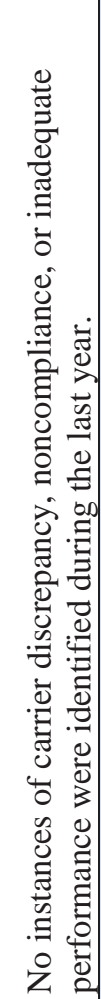 & 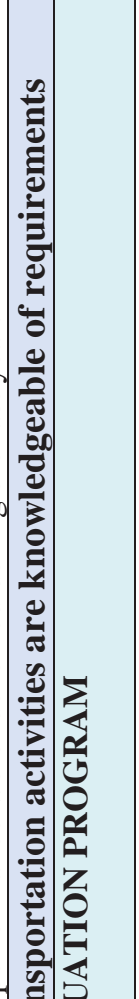 \\
\hline & & 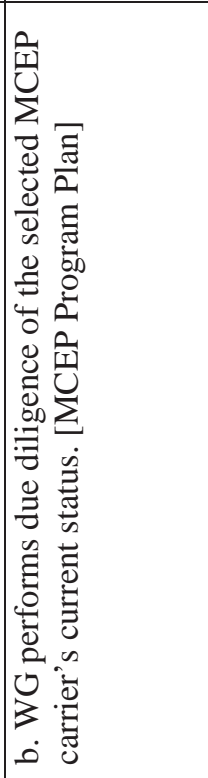 & & 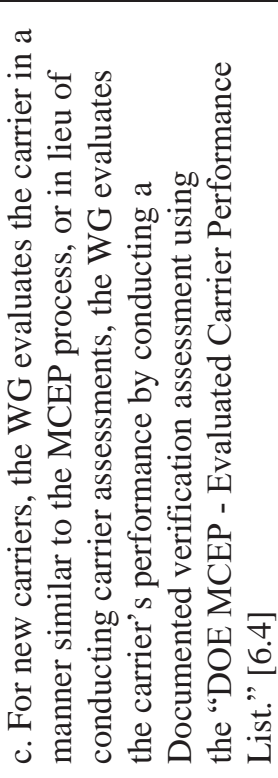 & 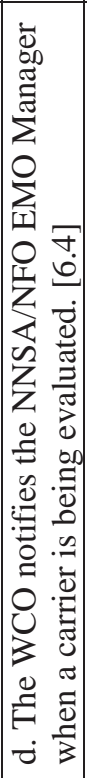 & 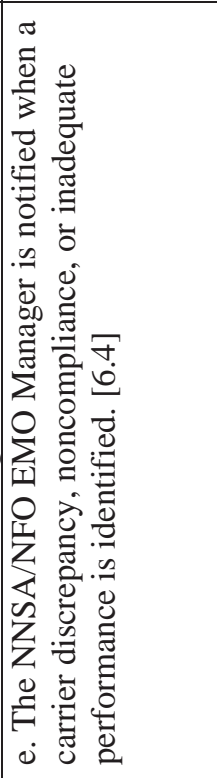 & & 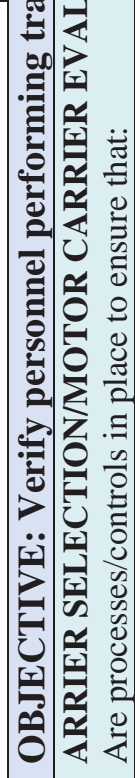 \\
\hline & & & & & & & & $m$ \\
\hline
\end{tabular}




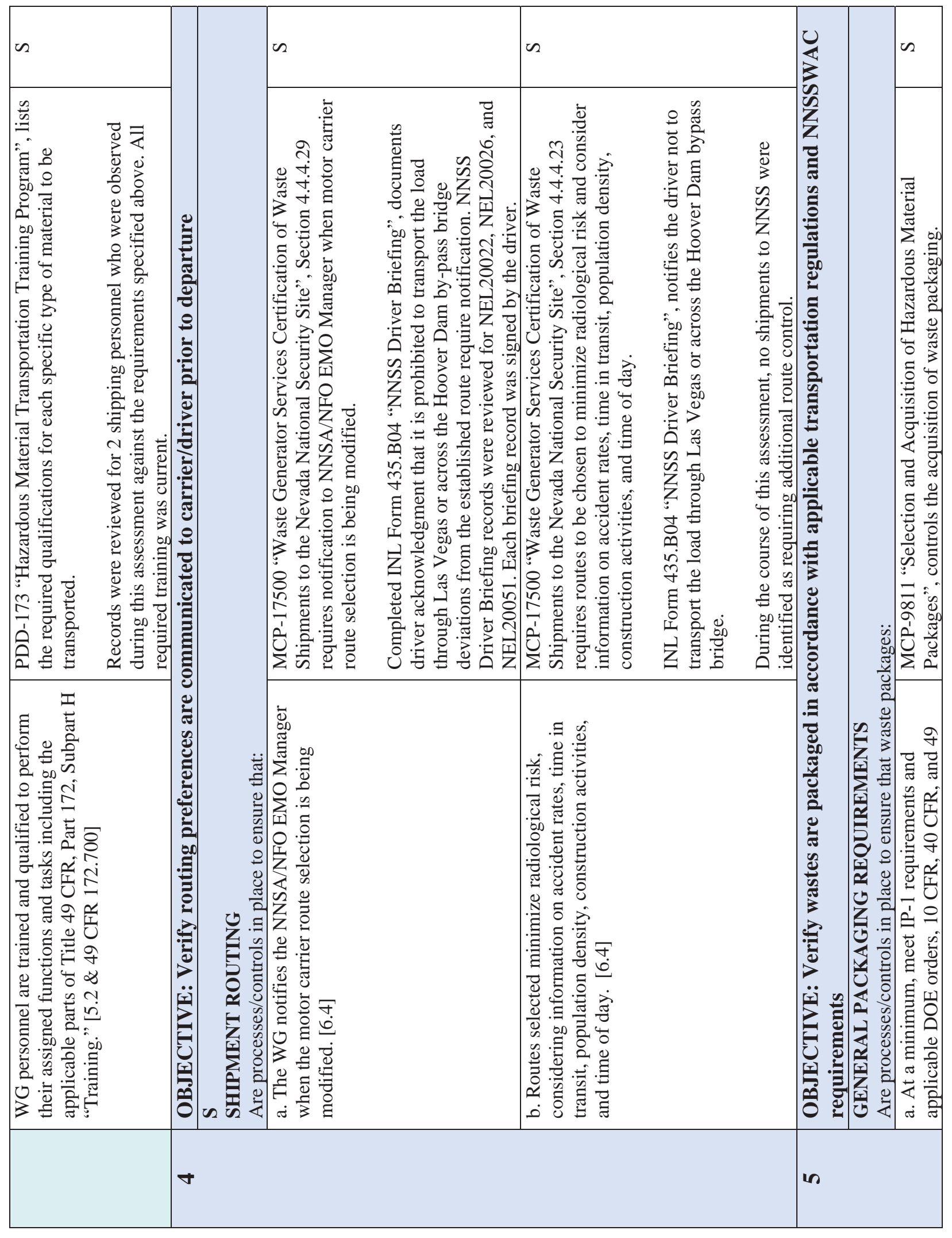




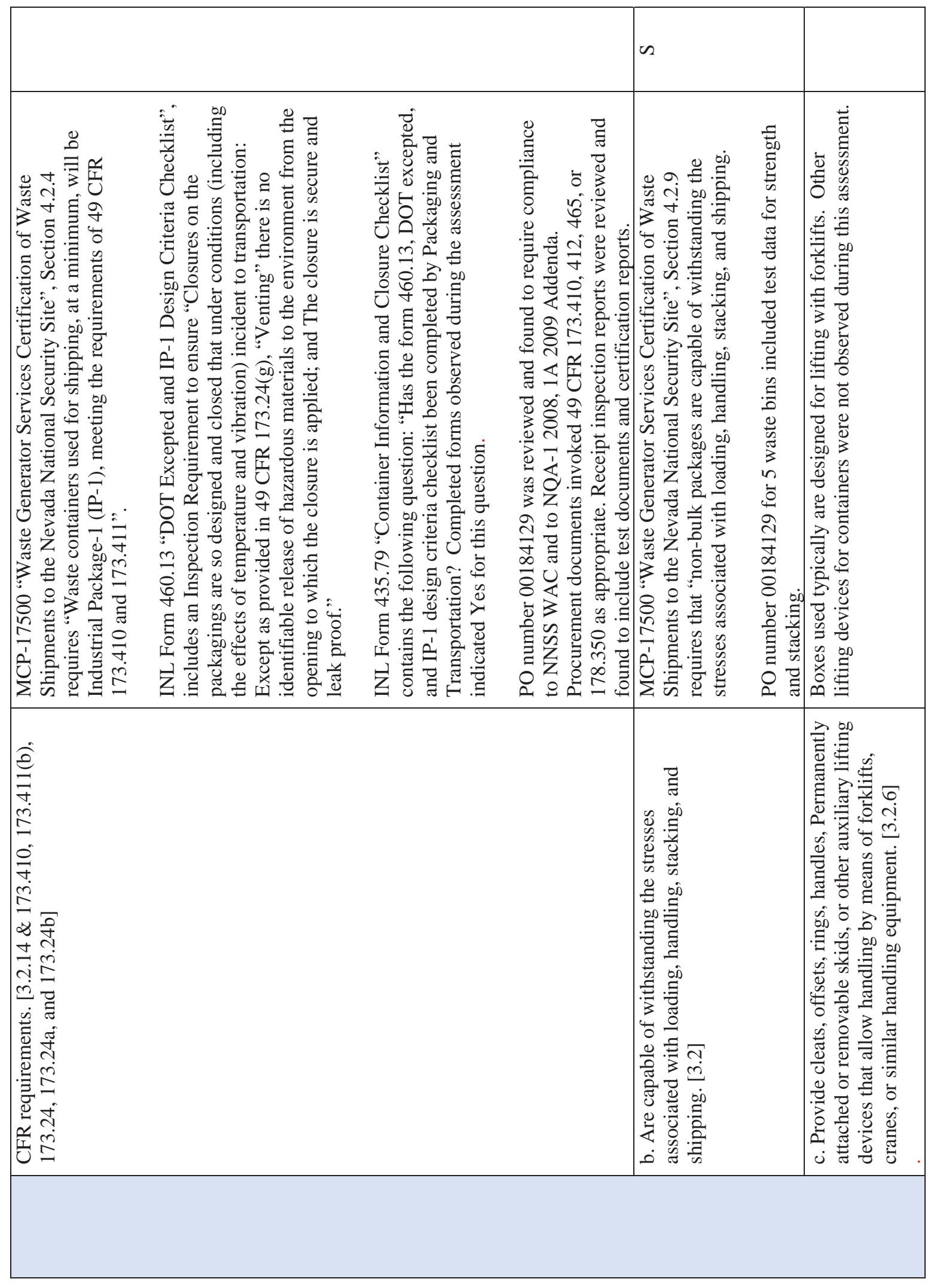




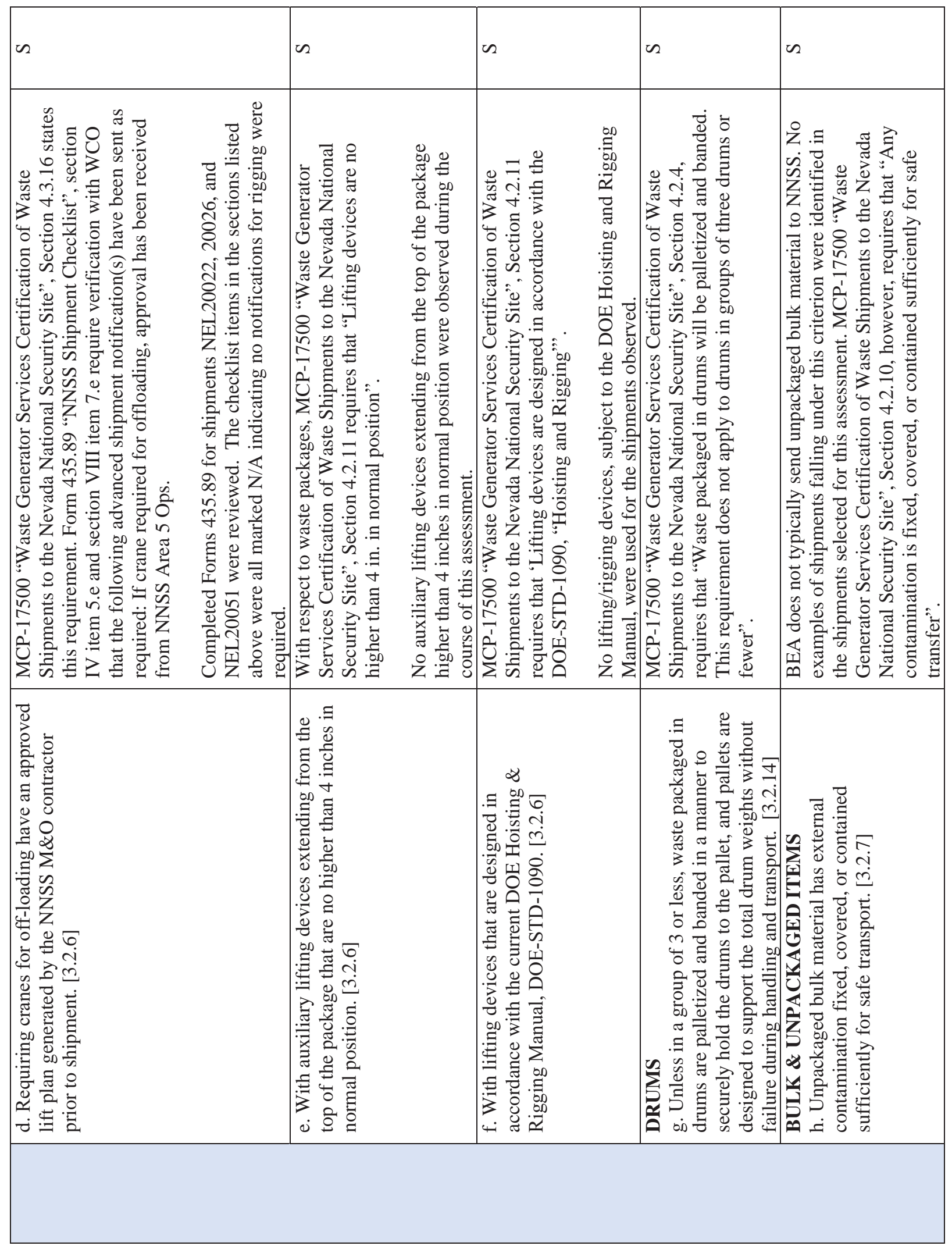




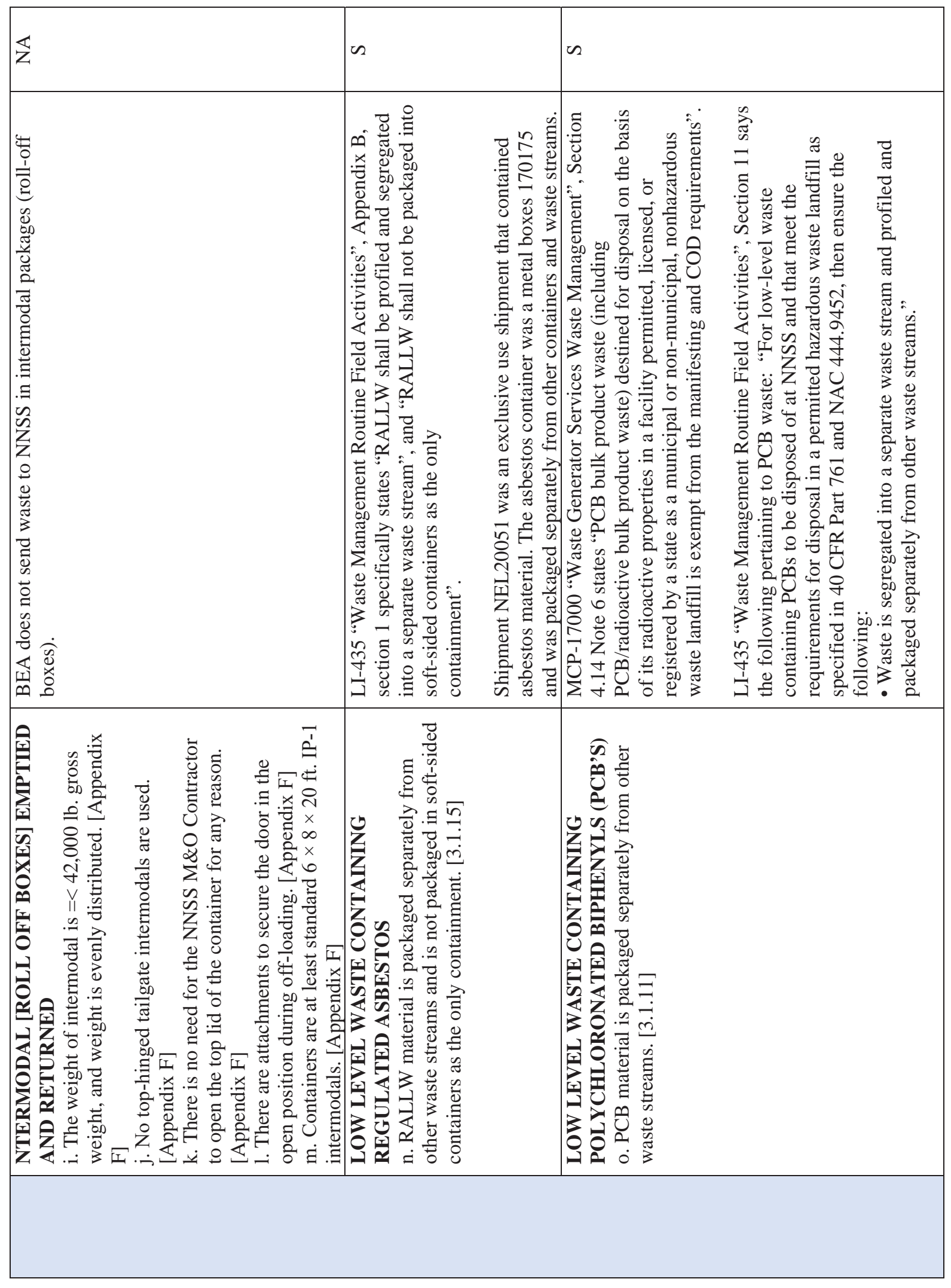




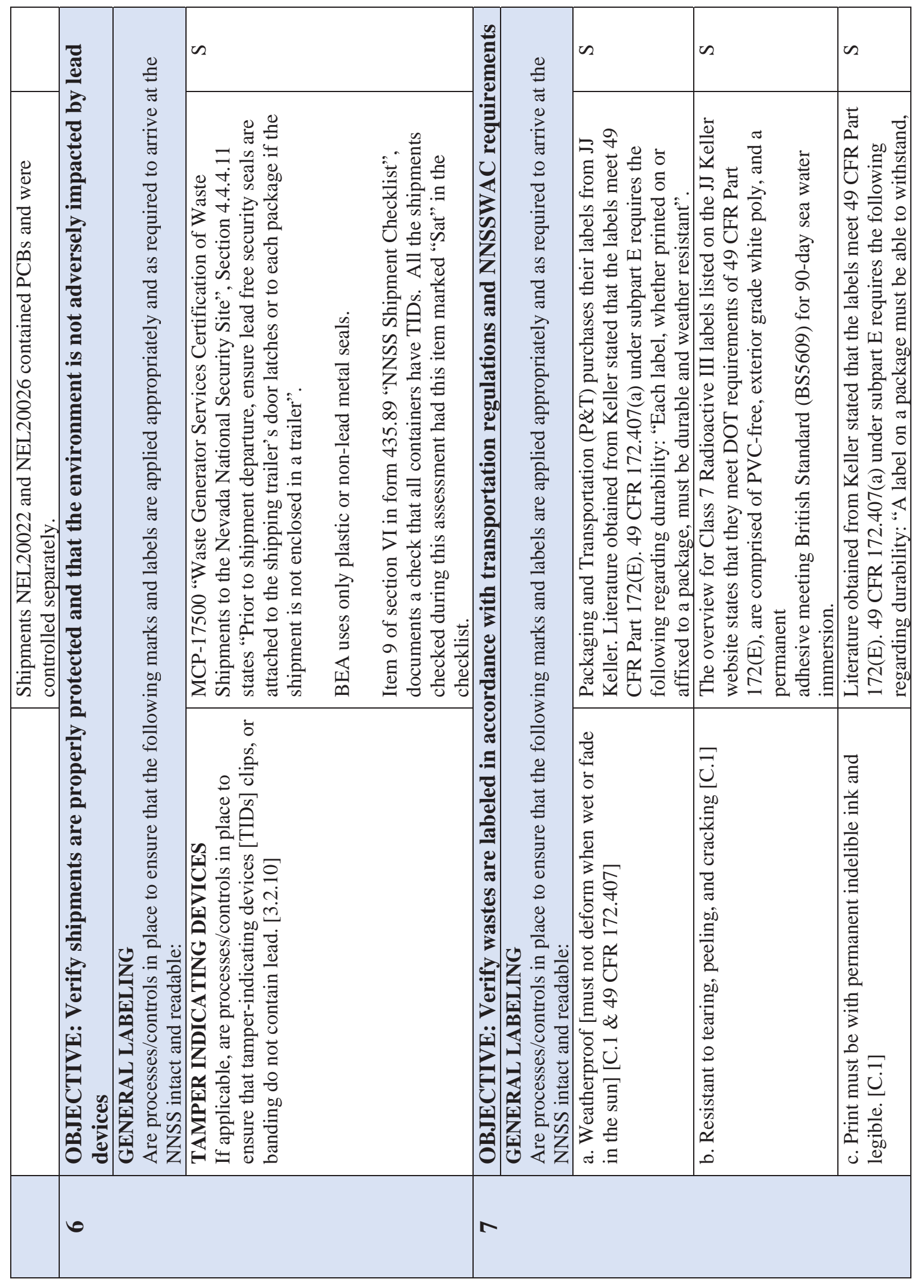




\begin{tabular}{|c|c|c|c|c|c|c|c|c|c|c|}
\hline & as & & & $n$ & & $\ln \mid \sin$ & us & & us & \\
\hline 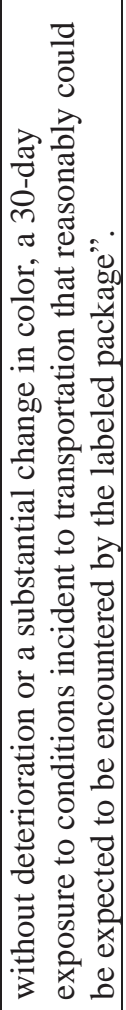 & 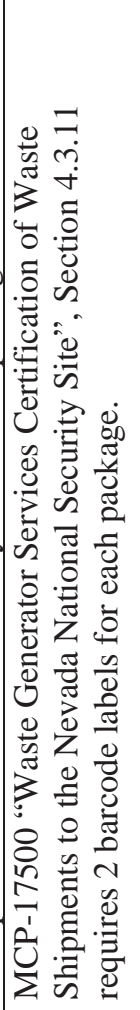 & 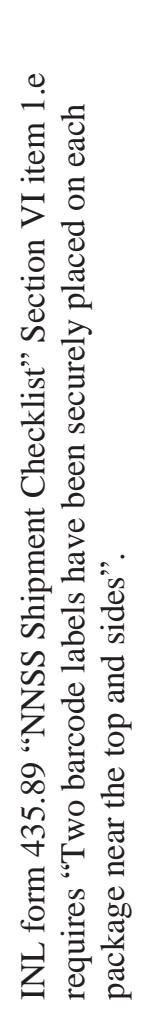 & 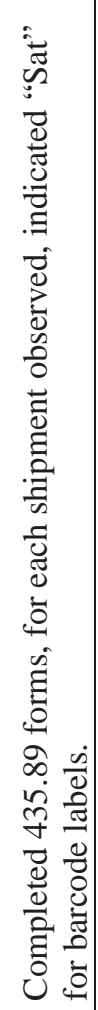 & 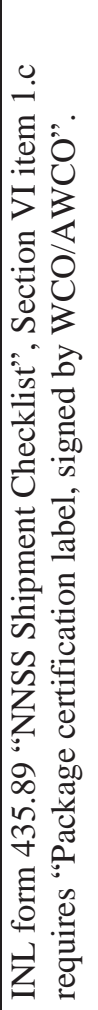 & 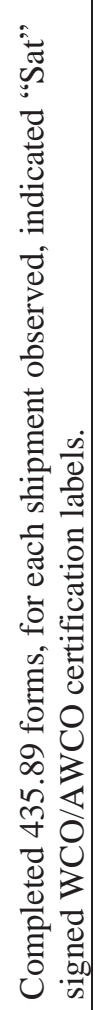 & 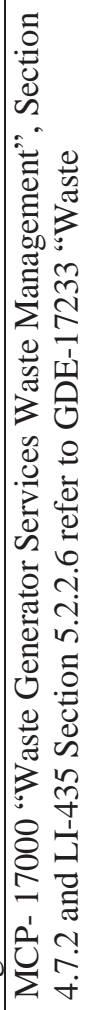 & 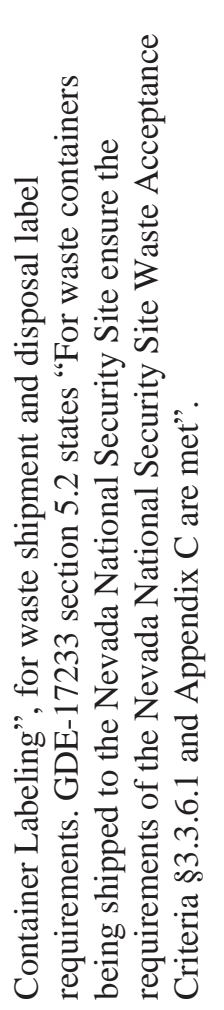 & 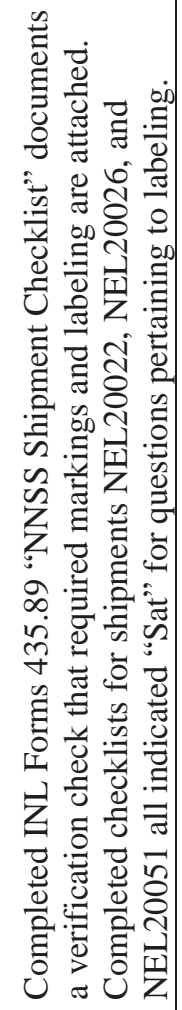 & 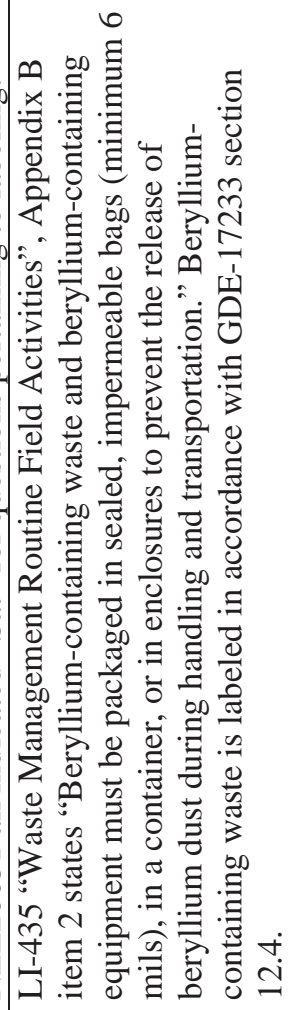 & 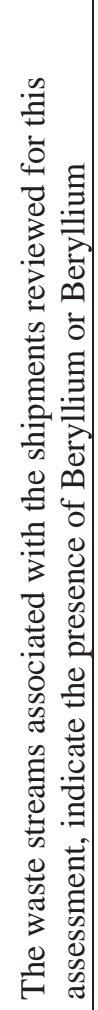 \\
\hline & 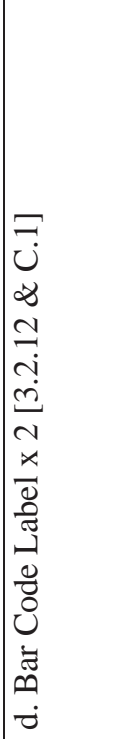 & & & 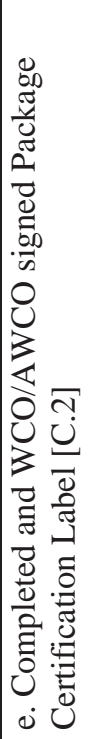 & & 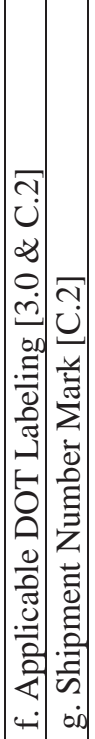 & 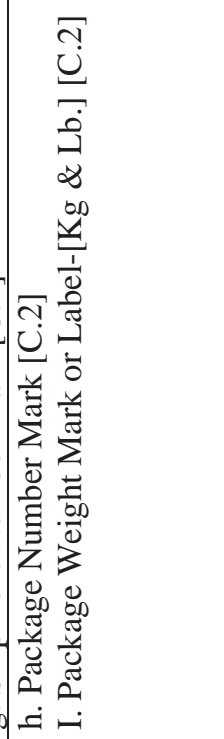 & & 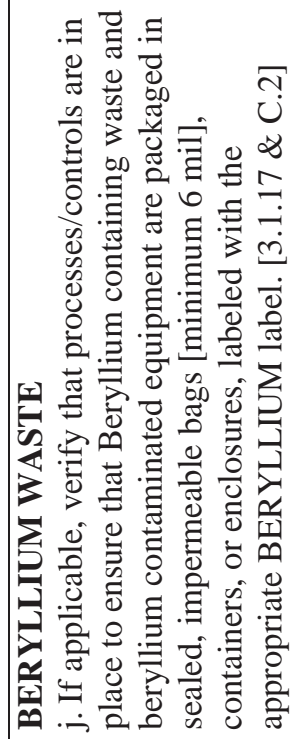 & \\
\hline
\end{tabular}




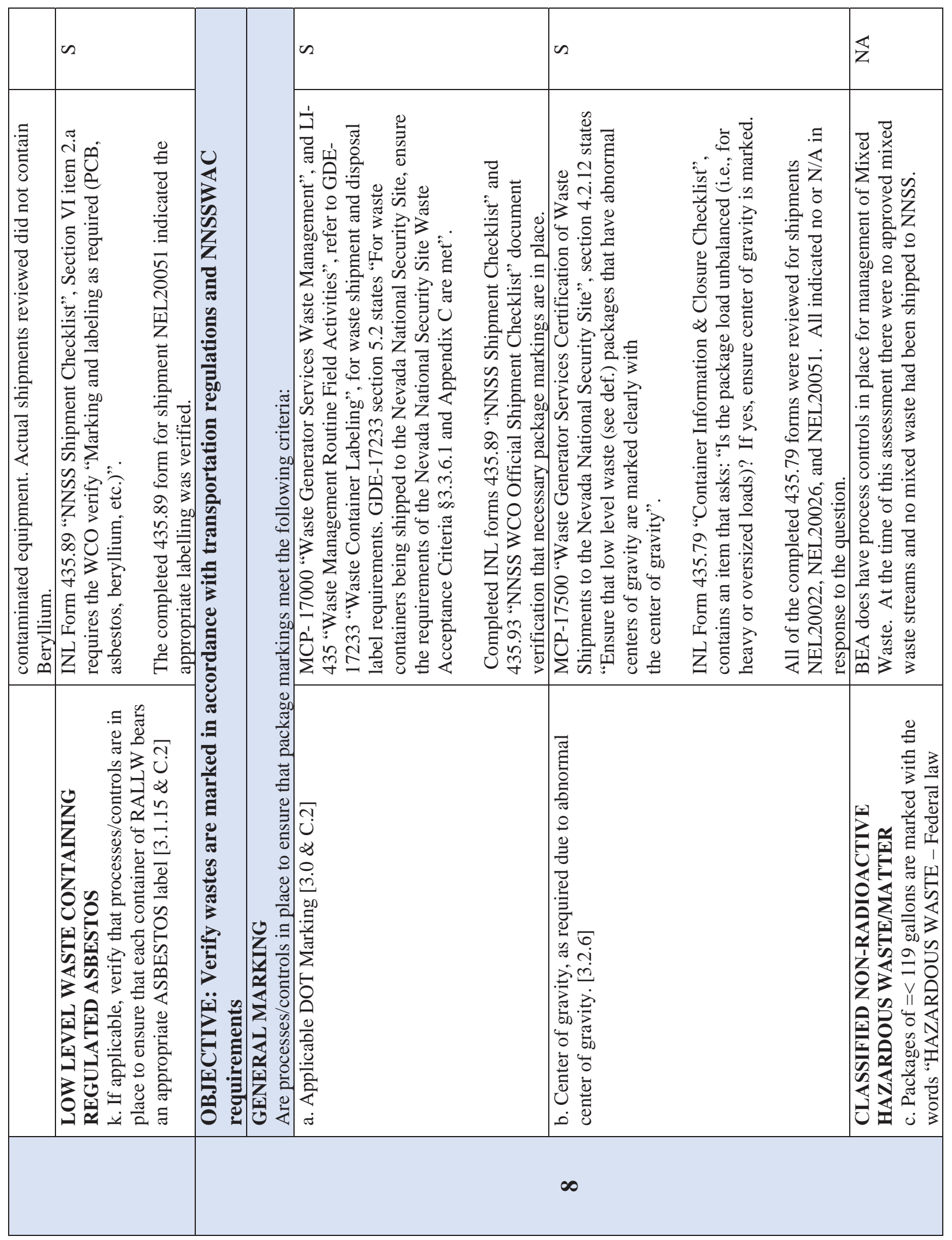




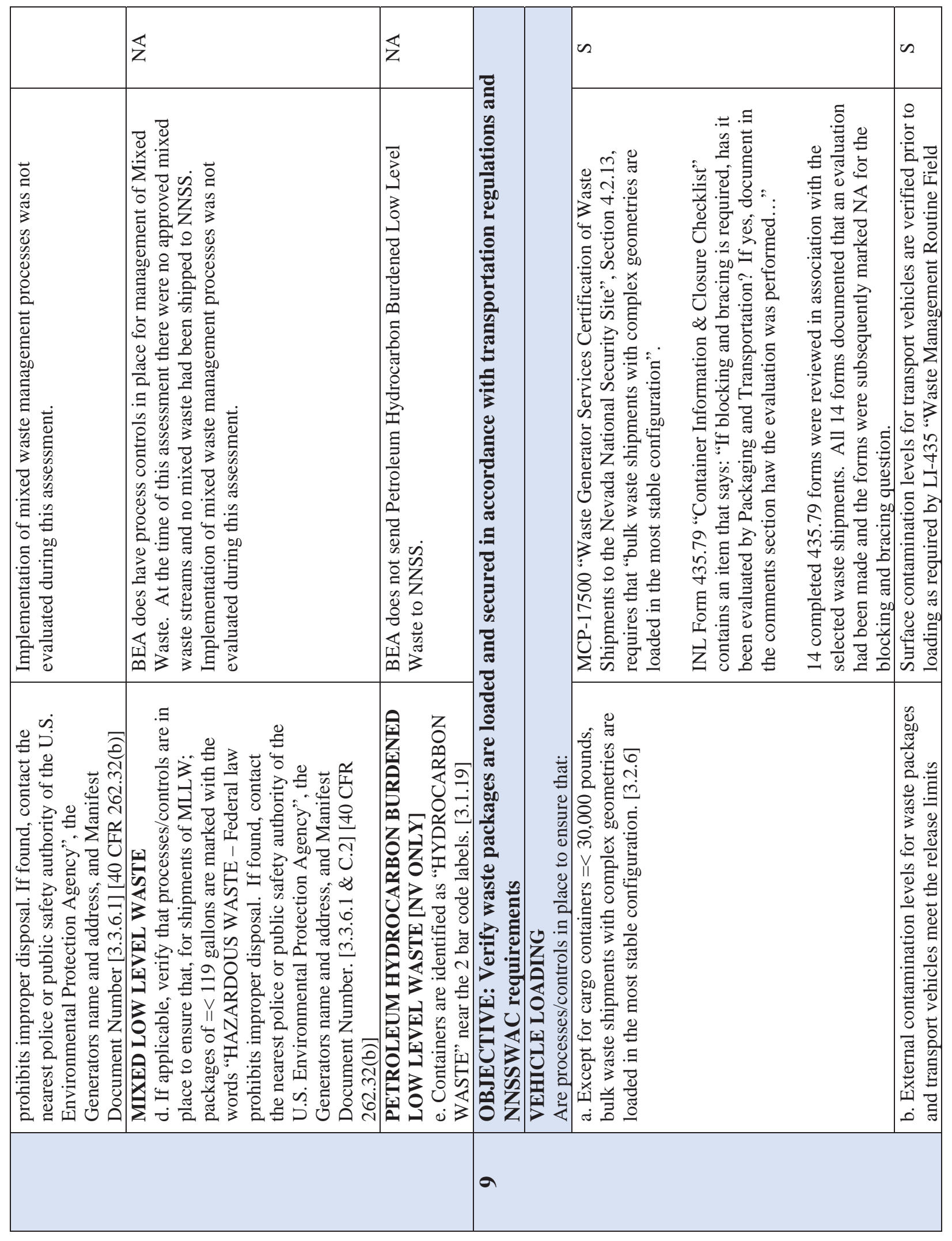




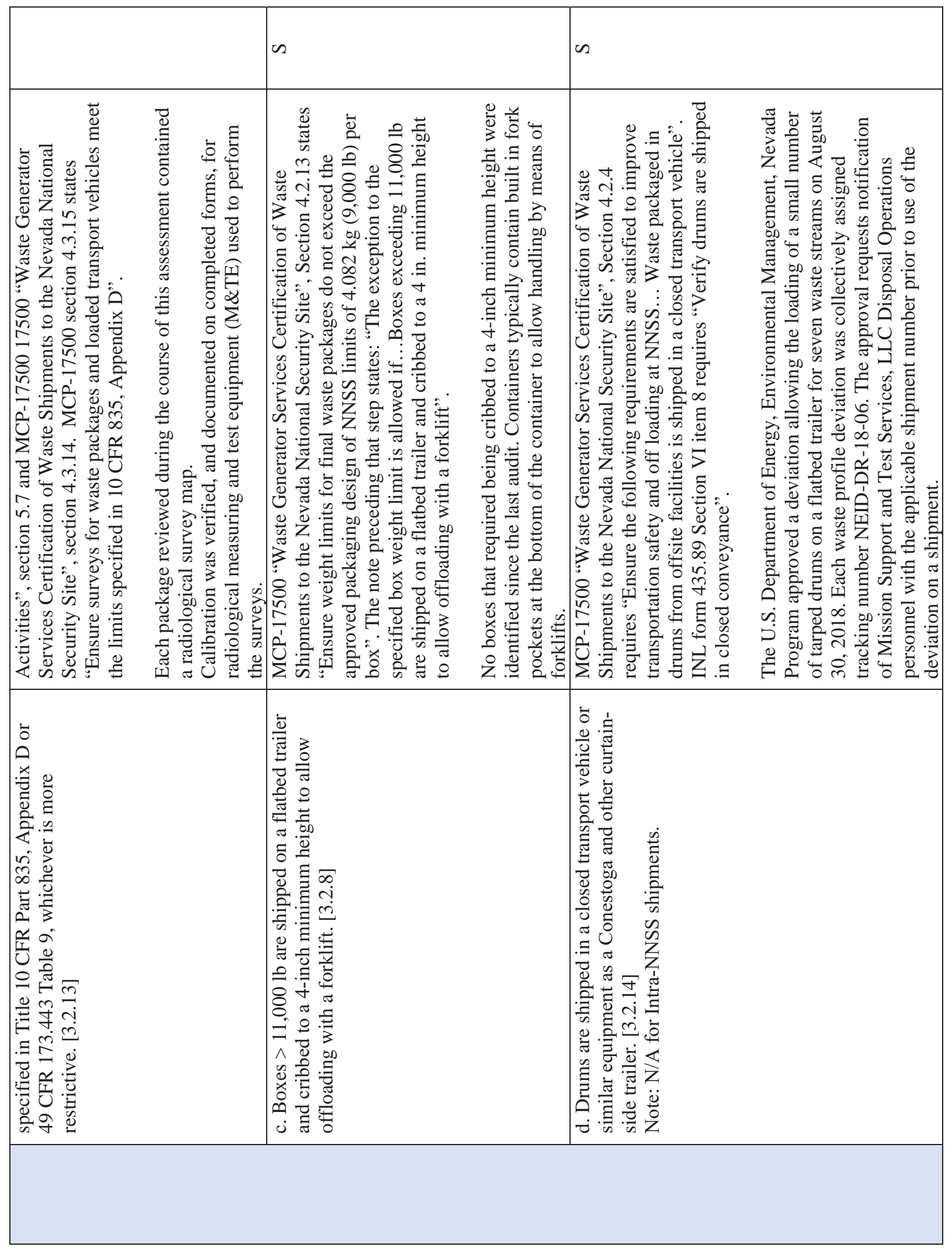




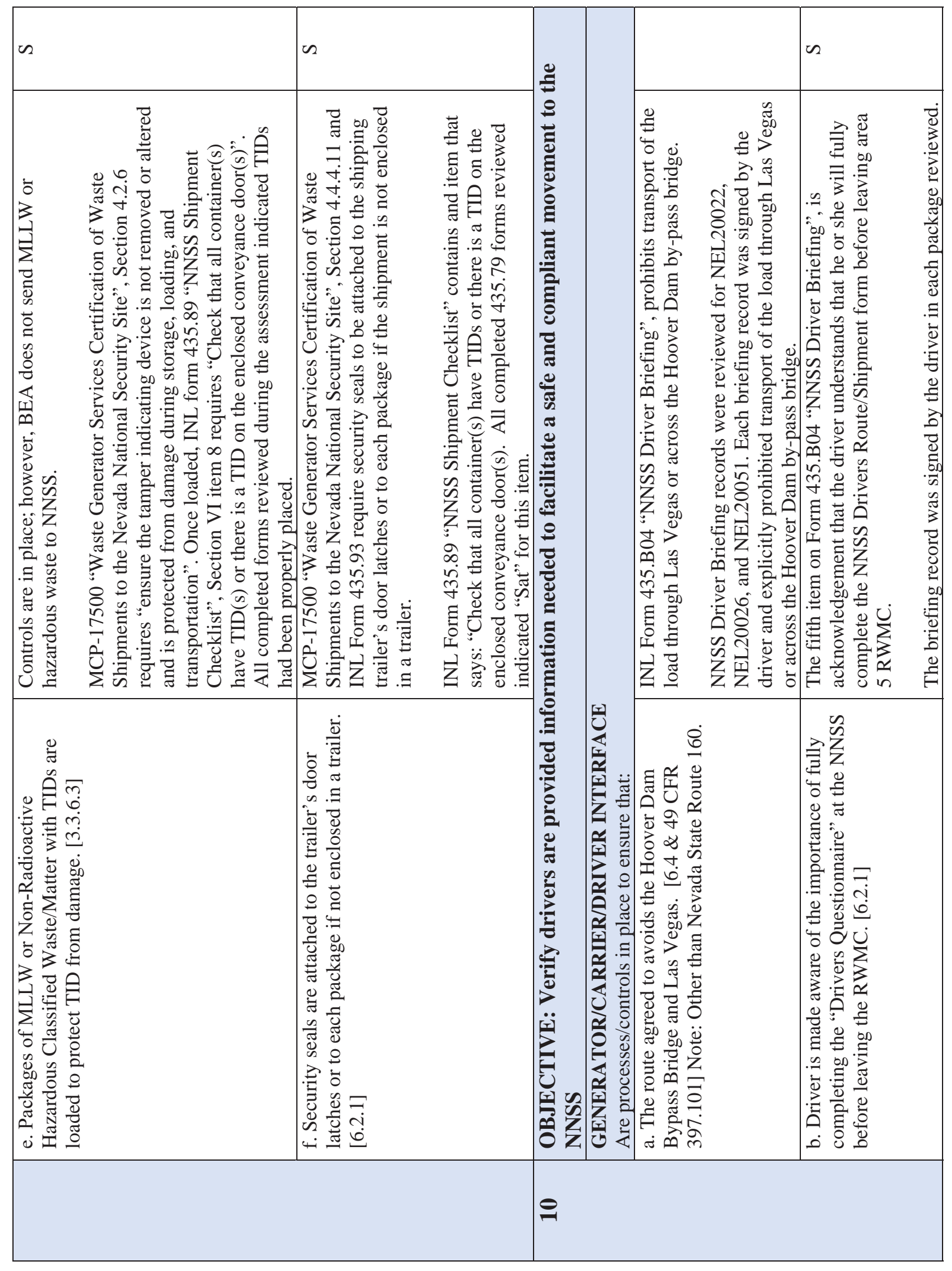




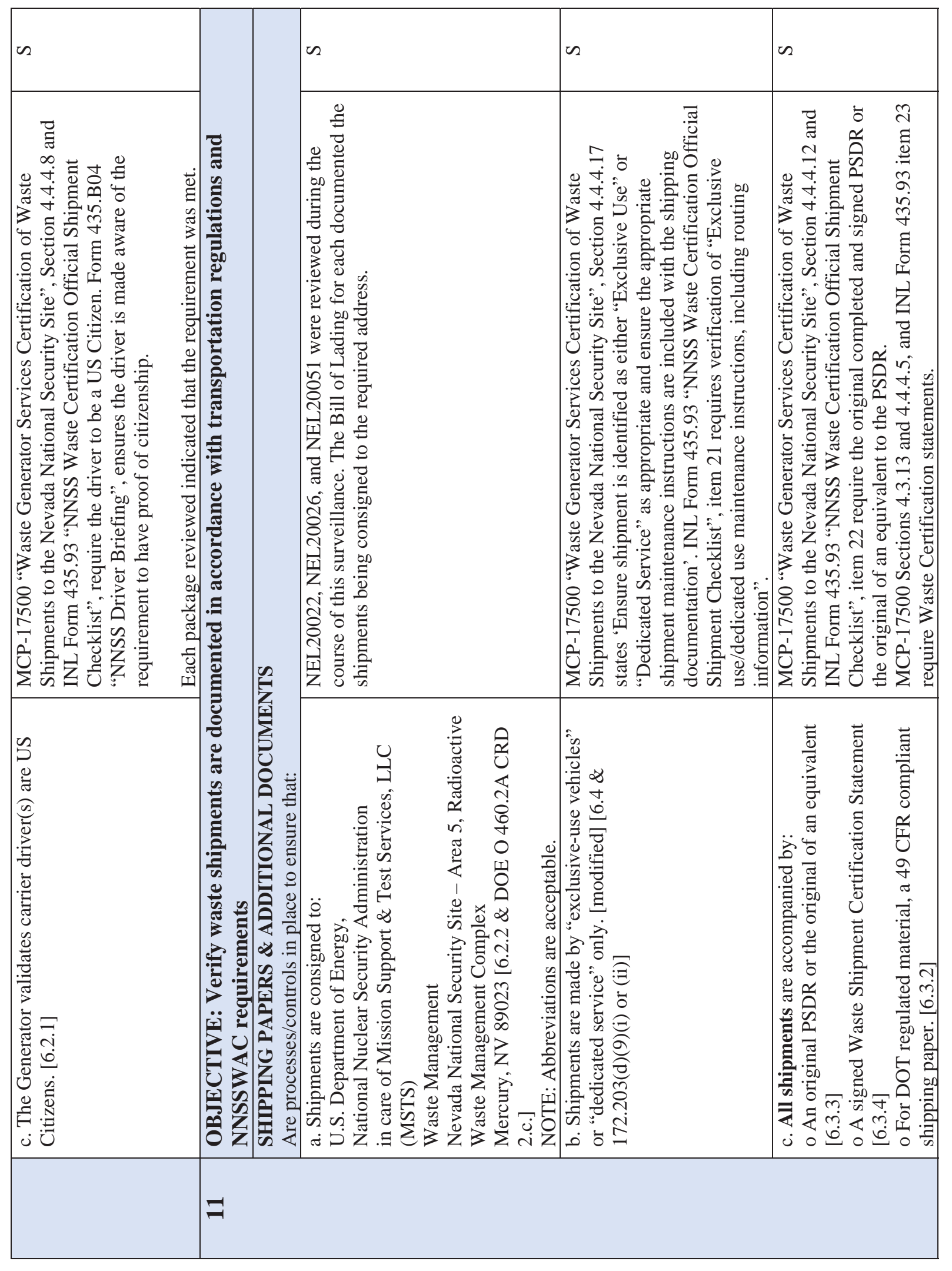




\begin{tabular}{|c|c|c|c|c|c|c|c|}
\hline & & os & & $\mathbb{Z}$ & $\mathbb{z}$ & es & \\
\hline \multirow[t]{2}{*}{ 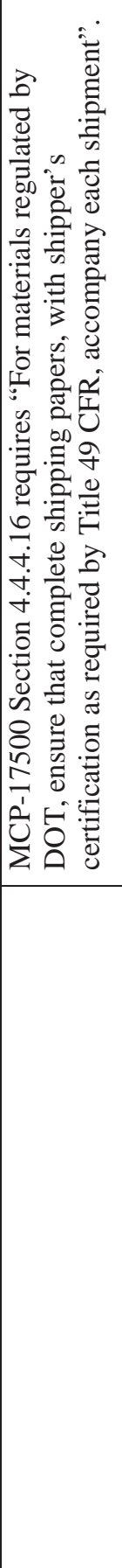 } & 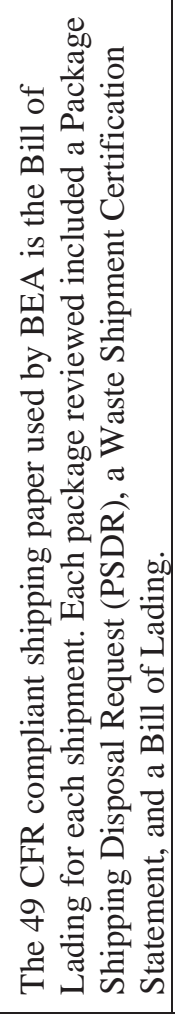 & 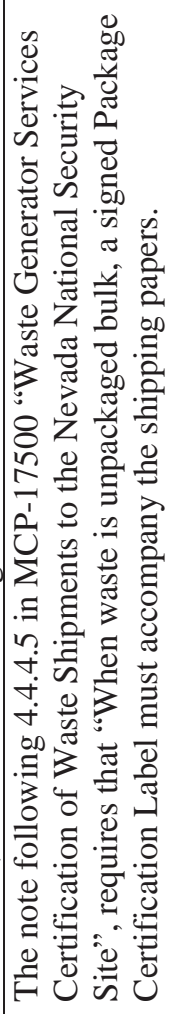 & 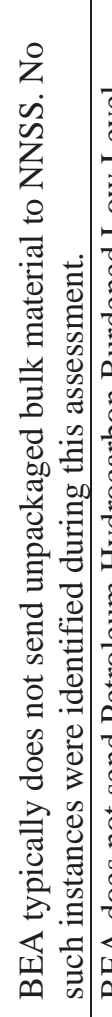 & 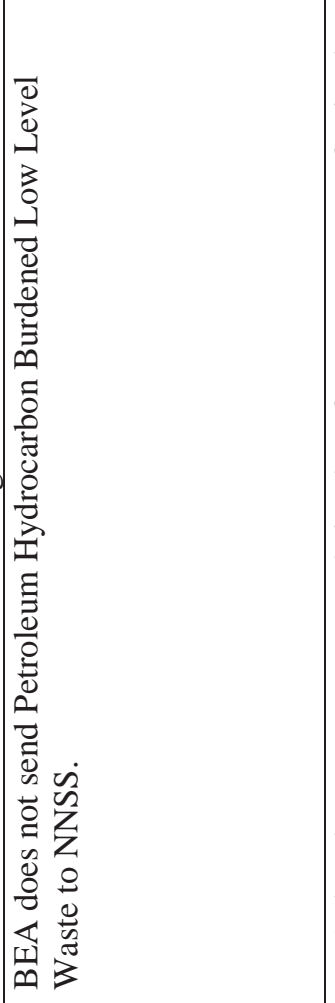 & 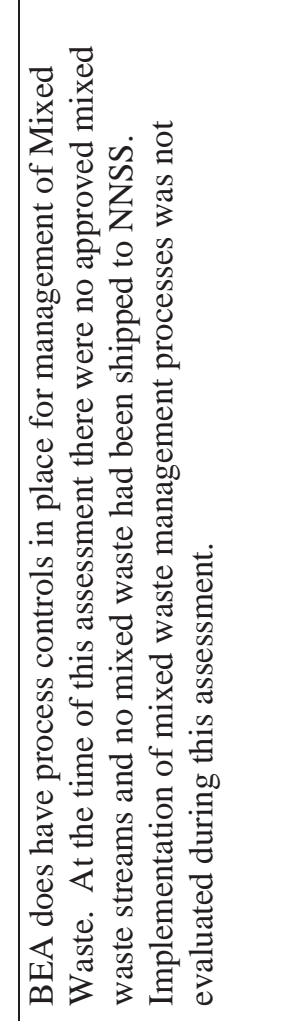 & \multicolumn{2}{|c|}{ 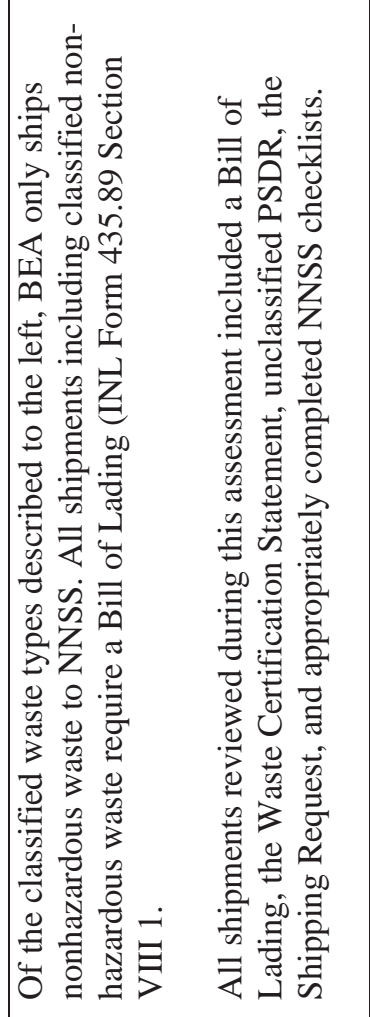 } \\
\hline & & 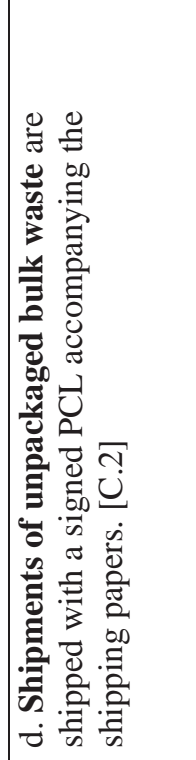 & & 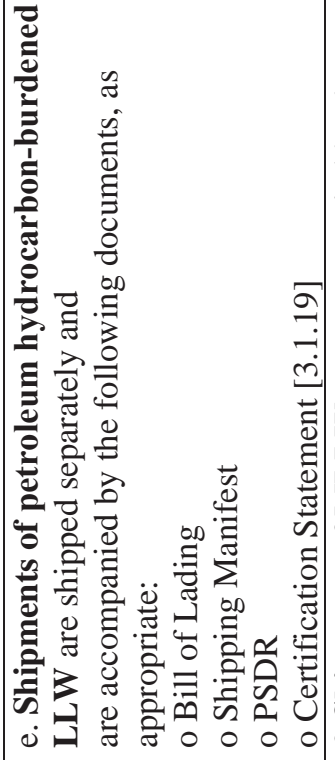 & 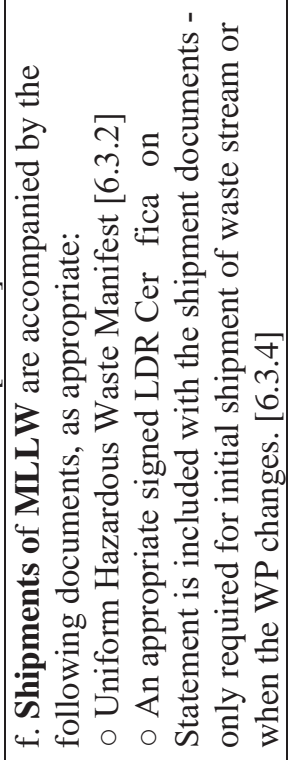 & 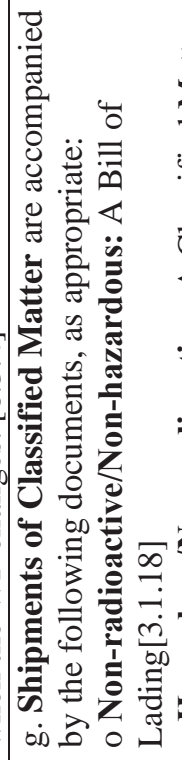 & 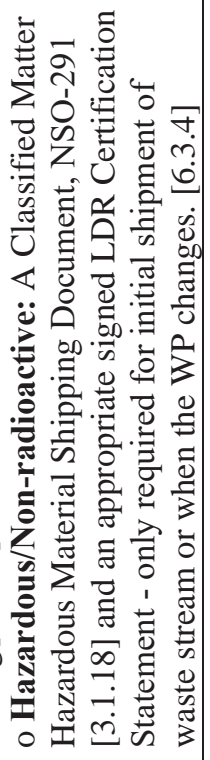 \\
\hline
\end{tabular}




\begin{tabular}{|c|c|c|c|c|c|c|c|}
\hline & 死 & $n$ & \multirow{3}{*}{ 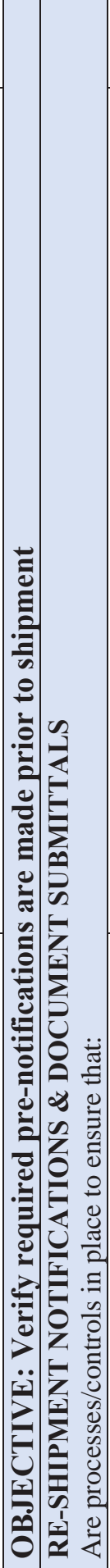 } & \multicolumn{3}{|l|}{ es } & as \\
\hline & 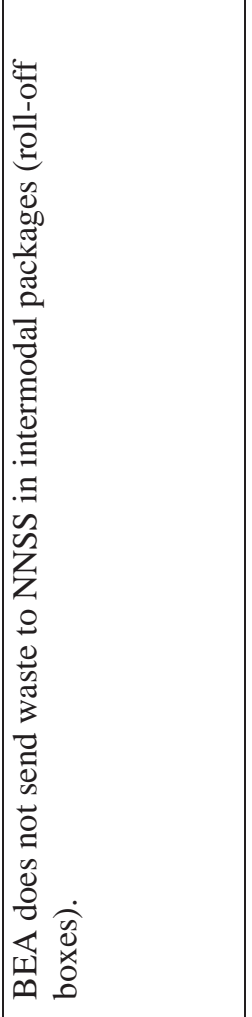 & 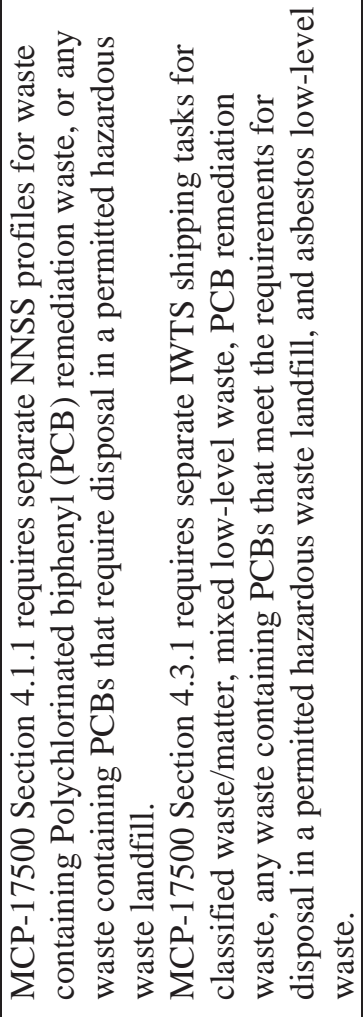 & & 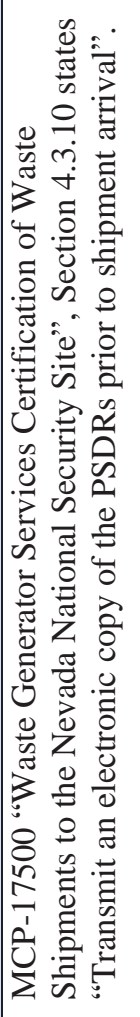 & 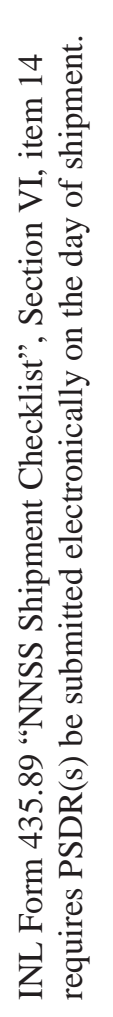 & 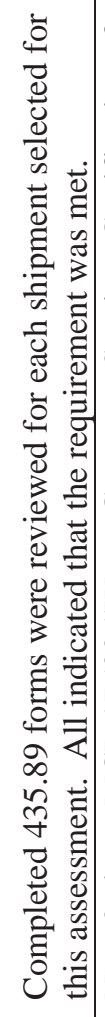 & 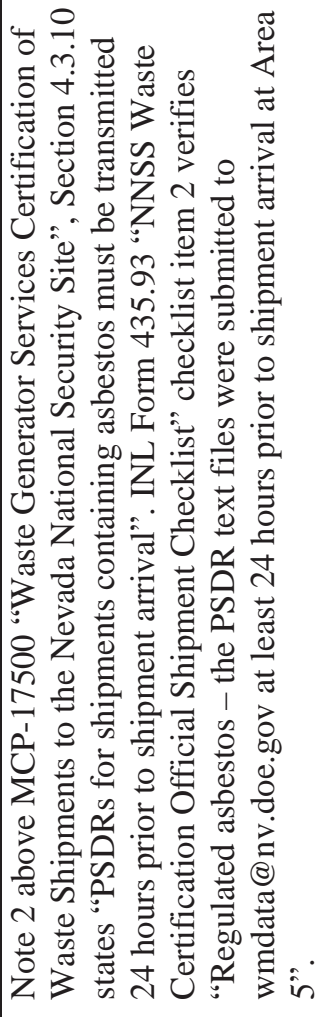 \\
\hline 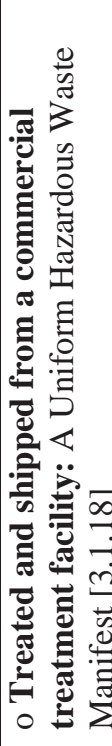 & 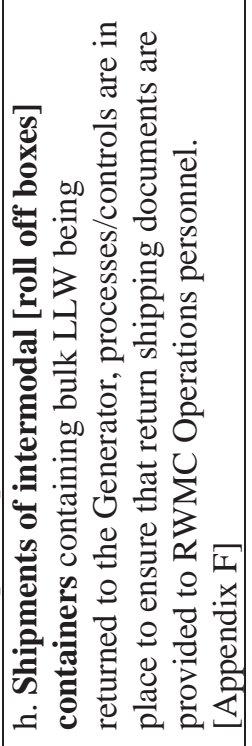 & 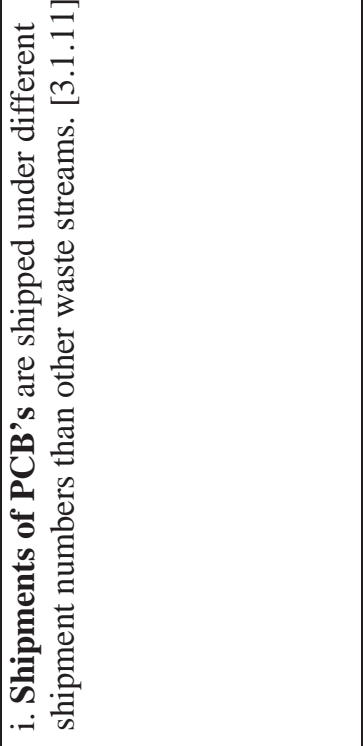 & & 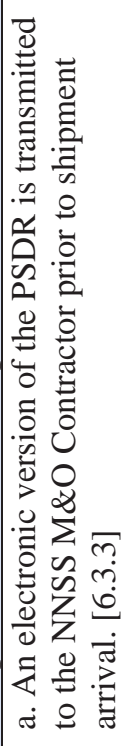 & & & 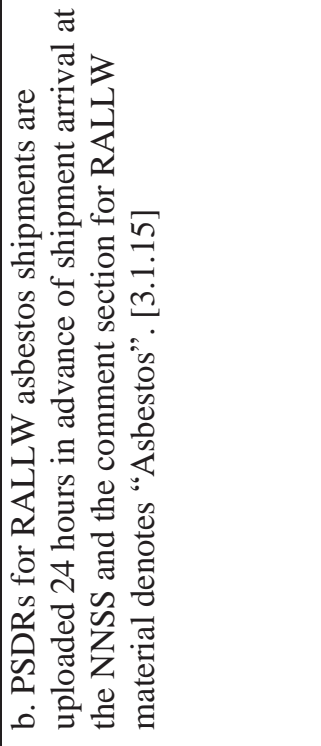 \\
\hline & & & $\approx$ & & & & \\
\hline
\end{tabular}




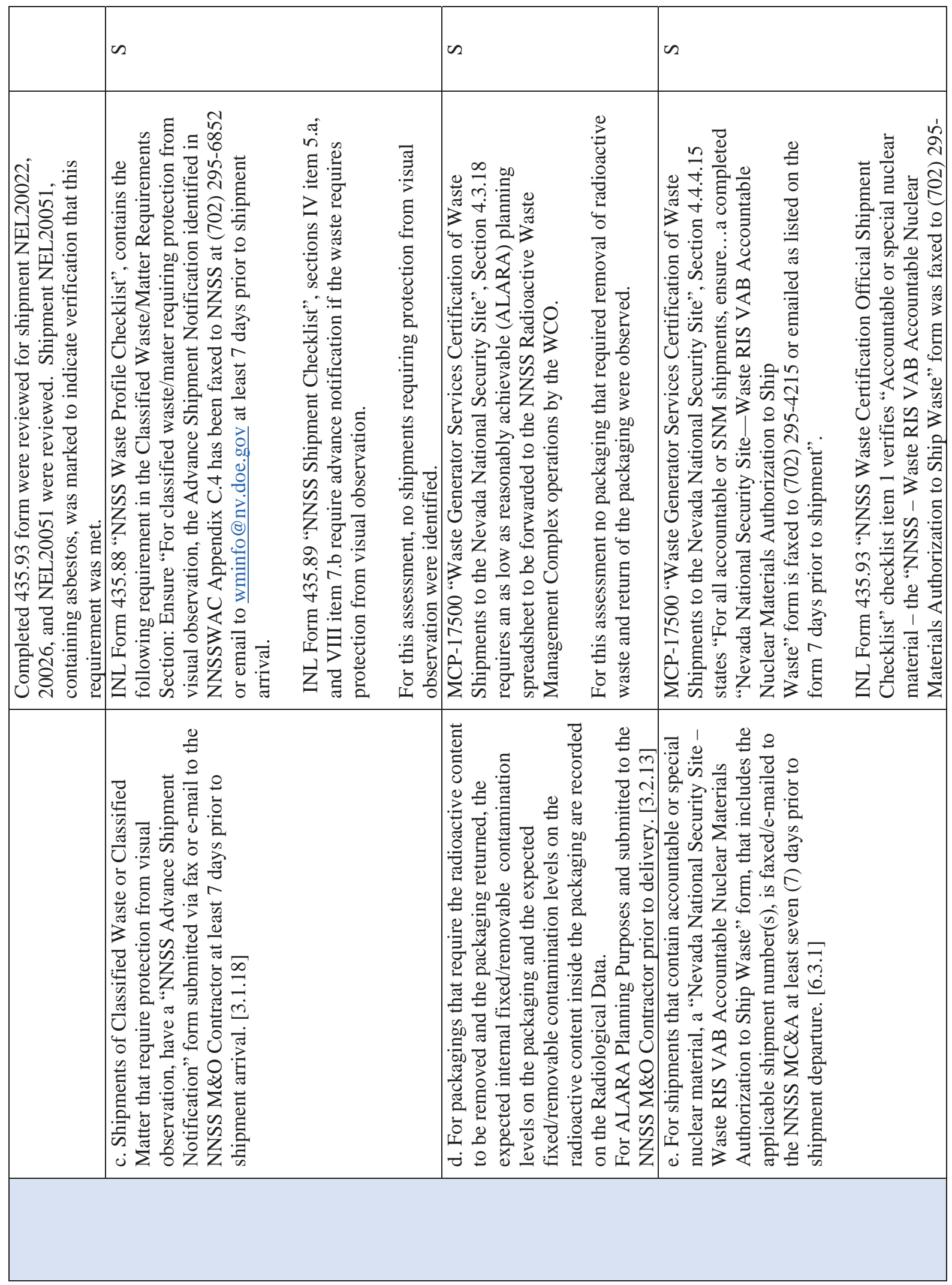




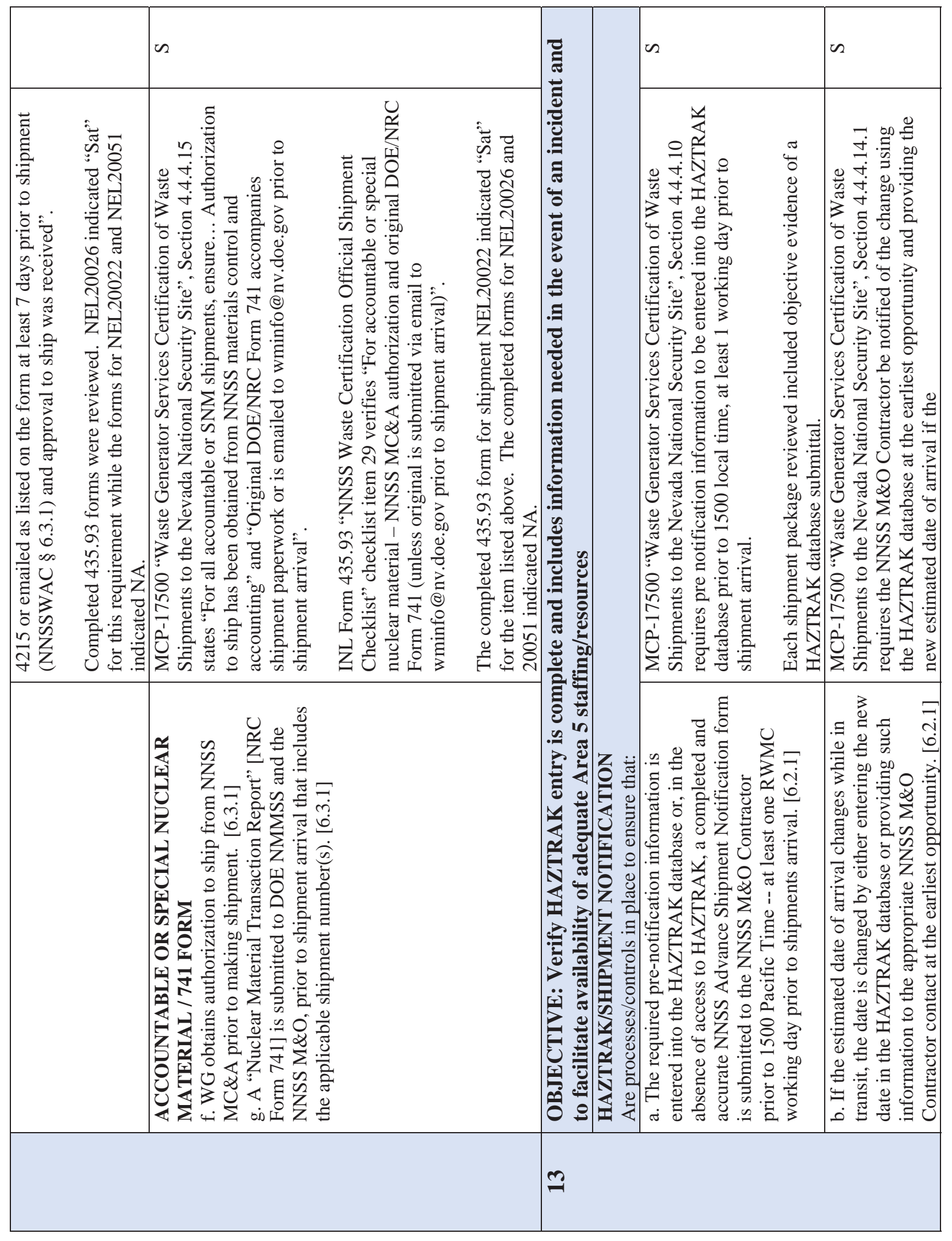




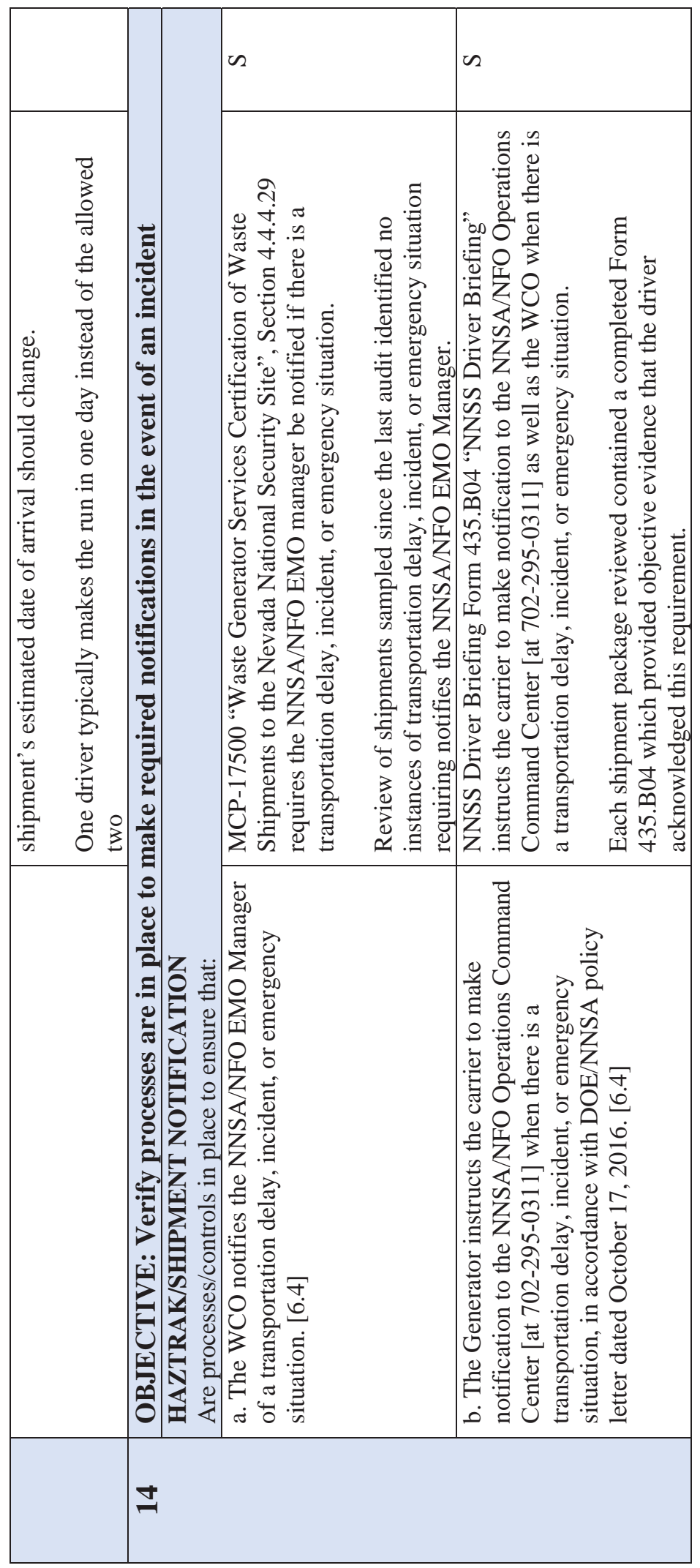

